\title{
A nonlocal approach of ductile failure incorporating void growth, internal necking, and shear dominated coalescence mechanisms
}

\author{
Van-Dung Nguyen ${ }^{\mathrm{a}}$, Thomas Pardoen ${ }^{\mathrm{b}}$, Ludovic Noels ${ }^{\mathrm{a}, *}$ \\ ${ }^{a}$ Computational \&3 Multiscale Mechanics of Materials, Department of Aerospace and Mechanical \\ Engineering, \\ University of Liège, Belgium \\ ${ }^{b}$ Institute of Mechanics, Materials and Civil Engineering, UCLouvain, Belgium
}

\begin{abstract}
An advanced modeling framework is developed for predicting the failure of ductile materials relying on micromechanics, physical ingredients, and robust numerical methods. The approach is based on a hyperelastic finite strain multi-surface constitutive model with multiple nonlocal variables. The three distinct nonlocal solutions for the expansion of voids embedded in an elastoplastic matrix are considered: a void growth phase governed by the Gurson-Tvergaard-Needleman yield surface, a void necking coalescence phase governed by a heuristic extension of the Thomason yield surface based on the maximum principal stress, and a competing void shearing coalescence phase triggered by the maximum shear stress. The first solution considers the diffused plastic deformation around the voids while the last two solutions correspond to a state of plastic localization between neighboring voids. This combination captures the Lode variable and shear effects, which play important roles in dictating the damage evolution rates. The implicit nonlocal formulation with multiple nonlocal variables, including the volumetric and deviatoric parts of the plastic strain, and the mean equivalent plastic strain of the matrix, regularizes the problem of the loss of solution uniqueness when material softening occurs whatever the localization mechanism. The predictive capability of the proposed model is demonstrated through different numerical simulations in which complex failure patterns such as slant and cup-cone of respectively plane strain and axisymmetric samples under tensile loading conditions develop.
\end{abstract}

Keywords: Ductile failure, Coalescence, Large strain, Nonlocal

\section{Introduction}

Ductile failure is controlled by the nucleation, growth, and coalescence of voids, together with extensive plastic dissipation accumulated before failure. A large number of predictive computational models has been developed in the literature to represent a physical description

*Corresponding author, Phone: +32436648 26, Fax: +3243669505

Email address: 1.noels @ ulg.ac.be (Ludovic Noels)

Preprint submitted to JMPS; (C)2020. Licensed under the Creative Commons (CC-BY-NC-ND)January 27, 2020 
of these mechanisms, see the reviews by Benzerga and Leblond (2010); Benzerga et al. (2016) and references therein.

The most popular ductile failure model was pioneered by Gurson (1977), with the socalled Gurson model, in which a macroscopic yield surface was derived from the homogenization of a porous material containing spherical voids embedded in an ideal elastoplastic matrix. The Gurson model introduces the void volume fraction into the macroscopic yield criterion to account for the material degradation. This original model can only predict the void growth phase. The Gurson model was subsequently extended in a semi-phenomenological form by Tvergaard and Needleman (Tvergaard and Needleman, 1984; Needleman and Tvergaard, 1984), with the so-called the GTN model, in order to represent the void nucleation and coalescence phases and to be in a better agreement with experimental measurements and void cell simulations. An important limitation of the GTN model is its inapplicability to correctly predict failure under low stress triaxiality. This limitation was resolved by Nahshon and Hutchinson (2008) with a Lode-dependent enhanced term in the void evolution. The GTN model has also been enriched in order to incorporate different characteristics of the voids, see the works by Gologanu et al. (1994, 1993); Pardoen and Hutchinson (2000); Benzerga et al. (2004); Keralavarma and Benzerga (2010). Although these latter models improve the physical description of the ductile failure process and better capture the low stress triaxiality failure conditions (Scheyvaerts et al., 2011), the incorporation of void shape, void orientation, and void rotation requires the calibration of a few more parameters (Pardoen, 2006). Therefore the original GTN framework is still preferred since its limited number of material parameters can be easily identified based on macroscopic mechanical tests, see the works of Springmann and Kuna (2005); Xue et al. (2010) for examples.

The void coalescence scenario depends on the loading conditions and on the spatial arrangement of voids. The most common is the internal necking coalescence mode, i.e. the shrinking of the ligament between neighboring voids along a localization band perpendicular to the main loading direction; the second is the shear coalescence mode, also called void sheeting, which occurs with the formation of micro shear bands inclined to the main loading direction and joining primary voids, possibly with secondary voids nucleated inside these micro bands. The rare necklace coalescence mechanism occurs between voids parallel to the main loading direction (Benzerga and Leblond, 2010). The void necking mechanism dominates at high stress triaxiality while the shear mechanism dominates at low stress triaxiality, favored by the presence of a second population of voids and a low strain hardening capacity (Pineau et al., 2016). Although the GTN model provides a complete computational methodology for all stages of void evolution, its phenomenological description of void coalescence, in which a critical value of the porosity is used to predict the onset of void coalescence beyond which the porosity growth rate is artificially accelerated through an effective porosity, does not provide a realistic description of void coalescence. On the one hand, the critical porosity is not a material constant but moderately depends on the stress state and on the initial porosity (Pardoen and Hutchinson, 2000; Kim et al., 2004; Gao et al., 2010). On the other hand, unit cell calculations under axisymmetric stress states reveal that the deformation mode during void coalescence is a pure uniaxial deformation (Pardoen and Hutchinson, 2000; Kim et al., 2004; Gao et al., 2010). This transition from a general pre-coalescence 
deformation mode to the uniaxial coalescence deformation mode cannot be correctly predicted using the GTN model. In order to overcome these limitations, micromechanics-based coalescence models pioneered by Thomason (1985a,b), with the so-called Thomason model, have been addressed and further elaborated over the last two decades.

The original Thomason model considers that coalescence starts when plastic deformation localizes in the ligaments between neighboring voids as supported by unit cell calculations (Pardoen and Hutchinson, 2000). This transition corresponds to a mode of plastic flow completely different than the one during the growth phase and not anymore correctly modeled with the GTN yield surface. This original version was further extended, see $e . g$ the works by Pardoen and Hutchinson (2000); Benzerga (2002); Scheyvaerts et al. (2010); Benzerga and Leblond (2014), to better represent the internal necking coalescence process. The Thomason model and its extended forms can be used either to detect the onset of coalescence while using the GTN framework (Zhang et al., 2000), or as an additional yield surface on equal footing with the GTN yield locus (so-called multi-surface approach) (Pardoen and Hutchinson, 2000; Benzerga et al., 2002; Pardoen and Hutchinson, 2003; Benzerga et al., 2004; Pardoen, 2006; Besson, 2009). However these works considered neither the effect of the Lode parameter in the yield condition, since the yield surface construction was based on axisymmetric configurations, nor the effect of the void shearing mechanism, although both of them play important roles in the ductile failure under general loading conditions as revealed by unit cell computations (Barsoum and Faleskog, 2011; Tekoğlu et al., 2012; Tekoğlu, 2014; Vadillo et al., 2016; Zhu et al., 2018) as well as by experiments (Bao and Wierzbicki, 2004; Barsoum and Faleskog, 2007).

Although the contribution of shear on the ductile failure process might be captured using the modified GTN model coupled with a phenomenological damage evolution taking into account the effect of shear on the damage evolution (Nahshon and Hutchinson, 2008; Xue, 2008), the underlying micro-mechanical basis is not well established since the failure process is no longer tied to an isotropic damage measure such as the void volume fraction. To address this issue, micro-mechanical-based onset conditions of void coalescence under combined tension and shear were proposed (Tekoğlu et al., 2012; Torki et al., 2015; Keralavarma and Chockalingam, 2016; Torki et al., 2017; Torki, 2019). Although these models represent better the physics of coalescence, their use in large scale simulations is still limited. On the one hand, they rely on complex evolution laws for the void characteristics such as void shape, relative void spacing, etc., so that the use of the GTN yield surface during the pre-coalescence stage is not appropriate. On the other hand, these complex models cannot easily be transformed into a yield surface representation. Recently, Reddi et al. (2019) provided an efficient way to derive a yield condition from the onset condition of void coalescence following the work of Keralavarma and Chockalingam (2016) by defining the orientation of the coalescence band as the combination of orientations of the principal stresses, leading to a multiple-yield surface plasticity model governing the coalescence process. This model considers the isotropic porosity to pilot the material degradation under the assumption that the void spacings in the transverse and axial directions are equal. However, this model predicts the onset of coalescence under pure shear at zero plastic strain because the coalescence surface always falls inside the Gurson yield surface under this loading condition. In addition, 
this model exhibits corners on the corresponding yield surface, leading to some difficulties from a computational point of view.

The present work focuses on building a constitutive model relying as much as possible on micromechanics grounds while making some empirical simplifications in the treatment of void growth and void coalescence in order to maintain an acceptable computational cost.

The first originality of the present approach is to consider the void growth, internal necking coalescence, and shear driven coalescence mechanisms in a combined form. Each void expansion solution (void growth, internal necking coalescence, and shear driven coalescence) is governed by its corresponding plastic problem. The competition between different void expansion solutions is obtained by defining a combined yield surface, leading to a multisurface approach. The void growth phase is governed by the Nahshon-Hutchinson version of the GTN yield surface (denoted by $\Phi_{\mathrm{G}}=0$ ) motivated by the good trade between a limited number of material parameters and predictive capabilities. During the void coalescence phase, the competition between void necking and void shearing mechanisms is considered. First, the internal necking mechanism is a heuristic extension (denoted by $\Phi_{\mathrm{T}}=0$ ) of the Thomason model and yield surface proposed by Pardoen and Hutchinson (2000). Since this mechanism is known to be governed by the maximum principal stress (MPS), the latter is then used as the controlling stress term in the Thomason yield surface. Next, under the assumption that the void shearing mechanism is governed by the maximum shear stress (MSS), a corresponding yield surface (denoted by $\Phi_{\mathrm{S}}=0$ ) is derived. As both maximum principal stress and maximum shear stress are two Lode-dependent quantities, the effect of the Lode parameter on the coalescence response is directly accounted for. The GTN, Thomason, and shear yield surfaces are recasted under a combined form, i.e. $\Phi_{\mathrm{e}}=\max \left(\Phi_{\mathrm{G}}, \Phi_{\mathrm{T}}, \Phi_{\mathrm{S}}\right)=0$, to govern the failure process. Moreover, a special treatment based on an approximation of the infinite norm is developed to avoid the corners of this combined form.

The second important element of the present approach is to work within a fully nonlocal setting. Most ductile failure models are formulated in the context of the local action principle, which implies that the damage indicator at one material point is estimated from the local variables at that point. When dealing with problems involving material strain softening, ellipticity is lost at the softening onset, resulting in strain localization in a band whose thickness depends on the mesh size. Consequently, the boundary value problem formulated in a standard local continuum becomes ill-posed and the finite element resolution becomes meaningless (De Borst et al., 1993). A simple remedy in engineering applications is to consider the size of the finite element mesh as an additional material parameter (Xue et al., 2010; Achouri et al., 2013). However, the choice of suitable finite element meshes is not an easy task because the material softening propagates at the level of the integration points and the localization band orientation depends on the element type and element direction. Moreover, the model reduction due to the symmetry of the boundary value problem cannot be exploited (Seidenfuss et al., 2011).

An extensive effort has been carried out to resolve the ill-posedness of the boundary value problem at the constitutive level by nonlocal formulations (Jirásek, 1998; Peerlings et al., 2001; Bažant and Jirásek, 2002) in which intrinsic lengths are incorporated into the constitutive relations allowing interactions between neighboring material points. This is 
achieved by assuming the dependence of the damage indicator on one or multiple nonlocal variables, which are estimated by volume averaging over a characteristic volume of their local counterparts. The dimensions of the characteristic volume then represent the intrinsic nonlocal lengths. Nonlocal extensions of the GTN model may be divided into three different categories: (i) integral type, see e.g. the works by Leblond et al. (1994); Tvergaard and Needleman (1995); Enakoutsa et al. (2007); Andrade et al. (2014), (ii) explicit gradient type, see e.g. the models by Chen and Yuan (2002); Zhang et al. (2018), and (iii) implicit gradient type, see e.g. the developments by Ramaswamy and Aravas (1998); Reusch et al. (2003b,a); Håkansson et al. (2006); Reusch et al. (2008); Linse et al. (2012); Hütter et al. (2013); Hütter et al. (2014); Zybell et al. (2014). The integral and implicit gradient types are found to be largely equivalent and referred to being strongly nonlocal while explicit gradient type can be considered as being weakly nonlocal (Peerlings et al., 2001). Besides, the explicit gradient type cannot be considered in the standard finite element implementation as it requires higher continuity (at least $\mathcal{C}^{1}$ ) of the nonlocal field (De Borst and Mühlhaus, 1992). Additionally, the ill-posed regularization can be achieved by coupling the elastoplastic models with the phase-field formulations, see e.g. the works of Ambati et al. (2016); Miehe et al. (2016); Aldakheel et al. (2018), to capture the highly localized failure stage as a result of coalescence of the voids. However, its underlying description remains phenomenological, in which some limitations have been previously pointed out. The implicit gradient type is employed in this work since it can be more easily integrated into a standard finite element implementation in comparison with the integral type (Peerlings et al., 1996). In the implicit gradient type, either the porosity (Ramaswamy and Aravas, 1998; Håkansson et al., 2006), the effective porosity (considered in the GTN model after applying the void expansion acceleration) (Reusch et al., 2003b,a, 2008), or the volumetric equivalent plastic strain (Linse et al., 2012; Hütter et al., 2013; Hütter et al., 2014; Zybell et al., 2014) is generally chosen as a nonlocal variable.

The difference between the nonlocal GTN model based on the porosity and the one based on the effective porosity is that the effective porosity is estimated from the nonlocal porosity in the first case while in the second case the effective porosity is itself a nonlocal variable estimated from its local counterpart. As shown by Jirásek and Rolshoven (2003), the nonlocal formulation based on the effective porosity results in an important expansion of the plastic zone with the transmitted stress stabilizing at a relatively high residual level, while the formulation based on the porosity provides more reasonable results. However, a constant length considered in a nonlocal formulation based on the damage indicator (the effective porosity in case of the GTN model) can lead to nonphysical results during the final failure process as pointed out by Jirásek (1998). Indeed if the nonlocal variable is chosen as the damage indicator, its local and nonlocal values must coincide at the last failure stage (Geers et al., 2000). This implies that the nonlocal length must vanish at final failure, requiring to consider its decrease with damage evolution (Poh and Sun, 2017).

Although the models considering the volumetric equivalent plastic strain as the nonlocal variable do not suffer from these pathologies, they fail to recover the solution uniqueness in all configurations. Indeed, the void evolution predicted by the GTN model is governed by three different sources: plastic incompressibility, nucleation, and shear deformation, which 
are respectively controlled by the volumetric equivalent plastic strain, matrix equivalent plastic strain, and the deviatoric equivalent plastic strain (Nahshon and Hutchinson, 2008). Note that the volumetric and deviatoric equivalent plastic strains are respectively defined from the first and second invariants of the plastic deformation tensor. Consequently, if an implicit gradient nonlocal model is based on only one of these three internal variables, the mesh-dependency might not be resolved if the void evolution is mainly controlled by one of the two other mechanisms. Moreover, the damage indicator in the coalescence phase is the void ligament ratio whose evolution is controlled not only by the porosity but also by the plastic dissipation (Besson, 2009). As a result, the nonlocal plastic deformation needs to be considered in order to provide an efficient regularization of the internal necking and shearing coalescence models. Therefore a nonlocal formulation must incorporate multiple sources as proposed in the context of viscoelastic-viscoplastic-damage law for polymer materials by Nguyen et al. (2016). For this purpose, the present approach is based on an implicit nonlocal model with multiple nonlocal variables (the volumetric equivalent plastic strain, the matrix equivalent plastic strain, and the deviatoric equivalent plastic strain) in order to achieve a full regularization of the multi-surface model. The complete approach constitutes a new step towards the development of ductile fracture frameworks relying on micromechanics and on a rigorous nonlocal treatment.

The paper is organized as follows. The governing equations of a boundary value problem with multiple nonlocal variables are first presented in Section 2 in a finite strain setting. In Section 3, the constitutive laws are developed for a general porous medium. The numerical fully-implicit integration scheme is provided. Next, this general framework is particularized to the multi-surface model in Section 4. In Section 5, we show that the proposed multisurface model can capture the effect of the stress triaxiality and of the Lode parameter under proportional loading conditions. Comparisons with unit cell simulations conducted under different triaxiality and Lode parameter states are also provided. Finally, the modeling framework is applied in Section 6 in order to demonstrate its ability to predict ductile failure in elastoplastic solids subjected to different stress states.

\section{Thermodynamically consistent nonlocal framework}

The loss of solution uniqueness in problems involving material softening can be efficiently addressed with the implicit gradient enhanced nonlocal approach pioneered by Peerlings et al. (1996). In this section, the governing equations derive from a thermodynamically consistent framework.

\subsection{Elastoplastic kinematics}

Let $B_{0}$ be a body with boundary $\partial B_{0}$. At time $t>0$, the deformed configuration $B$ is characterized by a two-point mapping $\mathbf{x}=\mathbf{x}(\mathbf{X}, t)$ where $\mathbf{x} \in B$ and $\mathbf{X} \in B_{0}$. This mapping determines the deformation gradient

$$
\mathbf{F}=\mathbf{x} \otimes \nabla_{0}=\mathbf{I}+\mathbf{u} \otimes \nabla_{0},
$$


where $\nabla_{0}$ is the spatial gradient operator with respect to the reference configuration $B_{0}$ and where $\mathbf{u}=\mathbf{x}-\mathbf{X}$ denotes the displacement vector. The Jacobian satisfies $J=\operatorname{det} \mathbf{F}>0$.

Following the standard multiplicative decomposition considered in elastoplastic materials (Moran et al., 1990), the deformation gradient $\mathbf{F}$ is decomposed as

$$
\mathbf{F}=\mathbf{F}^{\mathrm{e}} \cdot \mathbf{F}^{\mathrm{p}}
$$

where $\mathbf{F}^{\mathrm{e}}$ and $\mathbf{F}^{\mathrm{p}}$ represent, respectively, the elastic and plastic parts. One defines

$$
\mathbf{C}=\mathbf{F}^{T} \cdot \mathbf{F} \text { and } \mathbf{C}^{\mathrm{e}}=\mathbf{F}^{\mathrm{e} T} \cdot \mathbf{F}^{\mathrm{e}},
$$

as the right Cauchy strain tensor and its elastic counterpart, respectively.

From the decomposition of the deformation gradient following Eq. (2), the plastic spatial gradient of velocity is defined by

$$
\mathbf{L}^{\mathrm{p}}=\dot{\mathbf{F}}^{\mathrm{p}} \cdot \mathbf{F}^{\mathrm{p}-1}
$$

The tensor $\mathbf{L}^{\mathrm{p}}$ can be decomposed into its symmetric part $\mathbf{D}^{\mathrm{p}}$, so-called the plastic strain rate tensor, and its anti-symmetric part $\mathbf{W}^{\mathrm{p}}$, so-called the spin tensor, as $\mathbf{L}^{\mathrm{p}}=\mathbf{D}^{\mathrm{p}}+\mathbf{W}^{\mathrm{p}}$ with

$$
\mathbf{D}^{\mathrm{p}}=\frac{1}{2}\left(\mathbf{L}^{\mathrm{p}}+\mathbf{L}^{\mathrm{p} T}\right) \text { and } \mathbf{W}^{\mathrm{p}}=\frac{1}{2}\left(\mathbf{L}^{\mathrm{p}}-\mathbf{L}^{\mathrm{p} T}\right) .
$$

By assuming an irrotational plastic flow ${ }^{1}, \mathbf{W}^{\mathrm{p}}=\mathbf{0}$, one has

$$
\mathbf{L}^{\mathrm{p}}=\mathbf{D}^{\mathrm{p}}
$$

from which the evolution of the plastic deformation can be determined by

$$
\dot{\mathbf{F}}^{\mathrm{p}}=\mathrm{D}^{\mathrm{p}} \cdot \mathbf{F}^{\mathrm{p}}
$$

with $\mathbf{D}^{\mathrm{p}}$ specified from the plastic flow rule.

\subsection{Governing equations}

In this section, the generalized micromorphic framework suggested by Forest (2009) is adopted. At each material point, besides the deformation gradient $\mathbf{F}$ as the conventional kinematic variable, $N$ nonlocal mechanisms are considered with $N$ nonlocal variables $\bar{Z}_{k}$ with $k=1, \ldots, N$. The local generalized internal mechanical power is given by

$$
\mathcal{P}=\mathbf{P}: \dot{\mathbf{F}}+\sum_{k}\left(\bar{\sigma}_{k} \dot{\bar{Z}}_{k}+\overline{\mathbf{M}}_{k} \cdot \nabla_{0} \dot{\bar{Z}}_{k}\right)
$$

\footnotetext{
${ }^{1}$ For isotropic elastoplastic materials, when using the decomposition of the deformation gradient following Eq. (2), it is widely assumed (Gurtin and Anand, 2005; Anand et al., 2012), without loss of generality, that the plastic flow is irrotational $-i . e$. the plastic spin $\mathbf{W}^{\mathrm{p}}$ vanishes, since this simplifies the developments.
} 
where $\mathbf{P}$ denotes the first Piola-Kirchhoff stress, and $\bar{\sigma}_{k}$ and $\overline{\boldsymbol{M}}_{k}$ denote respectively the nonlocal source and the nonlocal flux of the nonlocal variable $\bar{Z}_{k}$. The stress measures P, $\bar{\sigma}_{k}$, and $\overline{\boldsymbol{M}}_{k}$ are energetically conjugate to the kinematic variables $\mathbf{F}, \bar{Z}_{k}$, and $\boldsymbol{\nabla}_{0} \bar{Z}_{k}$, respectively.

Over an arbitrary control volume $V_{0}$ with boundary $\partial V_{0}$ in the reference configuration $B_{0}$, the total internal power within this volume is given by

$$
W_{\mathrm{int}}=\int_{V_{0}} \mathcal{P} d V_{0}
$$

Without considering body force, the external power is expressed as

$$
W_{\mathrm{ext}}=\int_{\partial V_{0}}\left(\mathbf{T} \cdot \dot{\mathbf{u}}+\sum_{k} \bar{\zeta}_{k} \dot{\bar{Z}}_{k}\right) d A_{0}
$$

where $\mathbf{T}$ is the traction vector and $\bar{\zeta}_{k}$ is a scalar flux associated to the nonlocal variable $\bar{Z}_{k}$. Applying the Gauss's theorem on Eq. (9) and using the equality $W_{\text {int }}=W_{\text {ext }}$, which is satisfied for an arbitrary control volume $V_{0}$, lead to the equilibrium equation of the mechanical field and the ones of the nonlocal fields as

$$
\begin{aligned}
& \mathbf{P} \cdot \boldsymbol{\nabla}_{0}=\mathbf{0}, \text { and } \\
& \bar{\sigma}_{k}-\overline{\boldsymbol{M}}_{k} \cdot \boldsymbol{\nabla}_{0}=0 \text { with } k=1 \ldots N,
\end{aligned}
$$

and the traction vector and nonlocal scalar fluxes can be estimated from their stress measures by

$$
\begin{aligned}
& \mathbf{T}=\mathbf{P} \cdot \mathbf{N}, \text { and } \\
& \bar{\zeta}_{k}=\overline{\boldsymbol{M}}_{k} \cdot \mathbf{N} \text { with } k=1 \ldots N
\end{aligned}
$$

where $\mathbf{N}$ is the unit outward normal in the reference configuration.

The set of Eqs. $(11,12)$ defines the strong form of the boundary value problem. In order to complete the problem statement, on the one hand, the boundary conditions are applied on the boundary $\partial B_{0}$. For the displacement field $\mathbf{u}, \partial B_{0}$ is divided into two non-overlapping parts: a Neumann part $\partial_{N} B_{0}$ with prescribed traction $\overline{\mathbf{T}}$ and a Dirichlet part $\partial_{D} B_{0}$ with prescribed displacement $\overline{\mathbf{u}}$ as

$$
\left\{\begin{array}{l}
\mathbf{T}=\overline{\mathbf{T}} \text { on } \partial_{N} B_{0}, \text { and } \\
\mathbf{u}=\overline{\mathbf{u}} \text { on } \partial_{D} B_{0}
\end{array}\right.
$$

For the nonlocal fields $\bar{Z}_{k}$ with $k=1, \ldots, N$, it is assumed that no nonlocal fluxes stem from the external boundary $\partial B_{0}$ (Peerlings et al., 1996), i.e. $\bar{\zeta}_{k}=0$ with $k=1, \ldots, N$ on 
$\partial B_{0}$. As a result, Eq. (14) leads to

$$
\overline{\boldsymbol{M}}_{k} \cdot \mathbf{N}=0 \text { on } \partial B_{0} \text { with } k=1, \ldots, N .
$$

On the other hand, the required constitutive relationships connecting the stress measures $\mathbf{P}, \bar{\sigma}_{k}$, and $\overline{\boldsymbol{M}}_{k}$ to the kinematic variables $\mathbf{F}, \bar{Z}_{k}$, and $\boldsymbol{\nabla}_{0} \bar{Z}_{k}$ are derived from the second law of thermodynamics in the next section.

\subsection{Constitutive models}

Under isothermal condition, the Helmholtz free energy $\psi$ is assumed to depend on the elastic logarithmic strain measure $\mathbf{E}^{\mathrm{e}}=\ln \sqrt{\mathbf{C}^{\mathrm{e}}}$ (characterizing the elastic deformation state) and the nonlocal variables $\bar{Z}_{k}$ with $k=1, \ldots, N$ as well as their gradients $\nabla_{0} \bar{Z}_{k}$ by the following relationship

$$
\psi=\psi^{e}\left(\mathbf{E}^{\mathrm{e}}\right)+\sum_{k} \psi_{k}^{n l}\left(\bar{Z}_{k}, \nabla_{0} \bar{Z}_{k}\right)
$$

where $\psi^{e}$ is the reversible elastic part and the remaining term consists of the nonlocal parts driven by the nonlocal variables.

The reversible part $\psi^{e}$ depends on the elastic deformation measure only, which follows the usual assumption in porous media that the porosity does not significantly affect the elastic behavior as considered in numerous studies (Benzerga et al., 2016). The porosity and other characteristics of the voids are taken into account in the yield condition: during void expansion, the admissible region bounded by the yield surface in the stress space first extends with the diffused plastic deformation and then shrinks with material softening. The shrinkage of the yield surface corresponds to a decrease in the load carrying capacity (Engelen et al., 2003). The ultimate failure occurs when the yield surface shrinks to a point.

The nonlocal part of the nonlocal mechanism $k$ takes the following form (Peerlings et al., 2004)

$$
\psi_{k}^{n l}=h_{k}\left[\frac{\left(Z_{k}-\bar{Z}_{k}\right)^{2}}{2}+\frac{\nabla_{0} \bar{Z}_{k} \cdot \mathbf{C}_{k} \cdot \nabla_{0} \bar{Z}_{k}}{2}\right],
$$

where $Z_{k}$ is the local counterpart of the nonlocal variable $\bar{Z}_{k}, h_{k}$ is the coupling modulus, and $\mathbf{C}_{k}$ is the second order nonlocal length tensor associated with the nonlocal mechanism $k$. In this work, an isotropic nonlocal interaction is considered, so that $\mathbf{C}_{k}$ takes the following form

$$
\mathbf{C}_{k}=l_{k}^{2} \mathbf{I}
$$

where $l_{k}$ is the nonlocal length associated with the nonlocal mechanism $k$, and $\mathbf{I}$ is the second order identity tensor. In a general case, anisotropic nonlocal interaction can be obtained by introducing an anisotropic second order tensor as shown by Wu et al. (2013). Moreover, this work assumes that the material softening is driven by the irreversible process, therefore the 
local variables considered in Eq. (18) will be defined as functions of the internal variables related to the plastic deformation.

The Clausius-Duhem inequality requires the dissipation $\dot{\mathcal{D}}$ to be non-negative. In the isothermal cases, it reads

$$
\dot{\mathcal{D}}=\mathcal{P}-\rho_{0} \dot{\psi} \geq 0
$$

Using Eqs. (8, 17, 18), the condition Eq. (20) can be rewritten as

$$
\begin{aligned}
\dot{\mathcal{D}}= & {\left[\mathbf{P}:\left(\dot{\mathbf{F}}^{\mathrm{e}} \cdot \mathbf{F}^{\mathrm{p}}\right)-\rho_{0} \frac{\partial \psi^{e}}{\partial \mathbf{E}^{\mathrm{e}}}: \dot{\mathbf{E}}^{\mathrm{e}}\right]+\sum_{k}\left[\bar{\sigma}_{k}-\rho_{0} h_{k}\left(\bar{Z}_{k}-Z_{k}\right)\right] \dot{\bar{Z}}_{k} } \\
& +\sum_{k}\left[\left(\overline{\boldsymbol{M}}_{k}-\rho_{0} h_{k} \mathbf{C}_{k} \cdot \boldsymbol{\nabla}_{0} \bar{Z}_{k}\right) \cdot \nabla_{0} \dot{\bar{Z}}_{k}\right] \\
& +\left[\mathbf{P}:\left(\mathbf{F}^{\mathrm{e}} \cdot \dot{\mathbf{F}}^{\mathrm{p}}\right)-\sum_{k} \rho_{0} h_{k}\left(Z_{k}-\bar{Z}_{k}\right) \dot{Z}_{k}\right] \geq 0 .
\end{aligned}
$$

The condition (21) holds for all processes, leading to the following constitutive relationships

$$
\begin{aligned}
\mathbf{P} & =\mathbf{F}^{\mathrm{e}-T} \cdot \rho_{0} \frac{\partial \psi^{e}}{\partial \mathbf{E}^{\mathrm{e}}} \cdot \mathbf{F}^{\mathrm{p}-T}, \\
\bar{\sigma}_{k} & =\rho_{0} h_{k}\left(\bar{Z}_{k}-Z_{k}\right) \text { with } k=1, \ldots, N, \text { and } \\
\overline{\boldsymbol{M}}_{k} & =\rho_{0} h_{k} \mathbf{C}_{k} \cdot \nabla_{0} \bar{Z}_{k} \text { with } k=1, \ldots, N .
\end{aligned}
$$

As a result, the condition (20) reduces to

$$
\dot{\mathcal{D}}=\mathbf{P}:\left(\mathbf{F}^{\mathrm{e}} \cdot \dot{\mathbf{F}}^{\mathrm{p}}\right)-\sum_{k} \rho_{0} h_{k}\left(Z_{k}-\bar{Z}_{k}\right) \dot{Z}_{k} \geq 0
$$

Equation (22) can be rewritten as

$$
\mathbf{P}=\mathbf{F}^{\mathrm{e}-T} \cdot \boldsymbol{\tau} \cdot \mathbf{F}^{\mathrm{p}-T}
$$

where $\boldsymbol{\tau}$ is the stress measure energetically conjugate to $\mathbf{E}^{\mathrm{e}}$ with

$$
\boldsymbol{\tau}=\rho_{0} \frac{\partial \psi^{e}}{\partial \mathbf{E}^{\mathrm{e}}}
$$

Using Eq. (26), the Kirchhoff stress $\boldsymbol{\kappa}$ is given by

$$
\boldsymbol{\kappa}=\mathbf{P} \cdot \mathbf{F}^{T}=\mathbf{F}^{\mathrm{e}-T} \cdot \boldsymbol{\tau} \cdot \mathbf{F}^{\mathrm{e} T}
$$

The polar decomposition of the elastic deformation gradient tensor reads $\mathbf{F}^{\mathrm{e}}=\mathbf{R}^{\mathrm{e}} \cdot \mathbf{U}^{\mathrm{e}}$, where the symmetric part $\mathbf{U}^{\mathrm{e}}=\sqrt{\mathbf{C}^{\mathrm{e}}}$ is the right stretch tensor and $\mathbf{R}^{\mathrm{e}}$ is the orthogonal rotation tensor satisfying $\mathbf{R}^{\mathrm{e} T} \cdot \mathbf{R}^{\mathrm{e}}=\mathbf{I}$. Since $\boldsymbol{\tau}$ is a tensorial function of $\mathbf{U}^{\mathrm{e}}\left(\right.$ as $\left.\mathbf{E}^{\mathrm{e}}=\ln \sqrt{\mathbf{C}^{\mathrm{e}}}=\ln \mathbf{U}^{\mathrm{e}}\right)$ 
and considering an isotropic elastic potential in Eq. (27), one has $\boldsymbol{\tau} \cdot \mathbf{U}^{\mathrm{e}}=\mathbf{U}^{\mathrm{e}} \cdot \boldsymbol{\tau}$, and Eq. (28) can be rewritten as

$$
\boldsymbol{\kappa}=\mathbf{R}^{\mathrm{e}} \cdot \boldsymbol{\tau} \cdot \mathbf{R}^{\mathrm{e} T}
$$

From the last equation, $\boldsymbol{\tau}$ is interpreted as the Kirchhoff stress $\boldsymbol{\kappa}$ represented in the elastic corotational space. The Cauchy stress tensor $\boldsymbol{\Sigma}$ and its corotational counterpart $\boldsymbol{\sigma}$ are respectively estimated by

$$
\Sigma=\frac{\kappa}{J} \text { and } \boldsymbol{\sigma}=\frac{\tau}{J}
$$

Using Eqs. (23, 24), Eq. (12) can be rewritten as

$$
h_{k}\left[\bar{Z}_{k}-Z_{k}-\nabla_{0} \cdot\left(\mathbf{C}_{k} \cdot \nabla_{0} \bar{Z}_{k}\right)\right]=0, \text { with } k=1, \ldots, N .
$$

One can consider arbitrary non-zero values of $h_{k}$ with $k=1, \ldots, N$. In this case, the coupling modulus $h_{k}$ does not affect the relationship described by Eq. (31), leading to

$$
\bar{Z}_{k}-Z_{k}-\nabla_{0} \cdot\left(\mathbf{C}_{k} \cdot \nabla_{0} \bar{Z}_{k}\right)=0, \text { with } k=1, \ldots, N .
$$

The boundary conditions of the nonlocal variables following Eq. (16) are written as

$$
\left(\mathbf{C}_{k} \cdot \nabla_{0} \bar{Z}_{k}\right) \cdot \mathbf{N}=0 \text { on } \partial B_{0} \text { with } k=1, \ldots, N \text {. }
$$

If an isotropic nonlocal interaction is considered, i.e. $\mathbf{C}_{k}=l_{k}^{2} \mathbf{I}$ with $k=1, \ldots, N$, the usual natural boundary conditions for nonlocal variables (Peerlings et al., 1996) are recovered

$$
\nabla_{0} \bar{Z}_{k} \cdot \mathbf{N}=0 \text { on } \partial B_{0} \text { with } k=1, \ldots, N .
$$

In the following, one can define the nonlocal vector $\overline{\mathbf{Z}}$ and its local counterpart $\mathbf{Z}$ respectively as

$$
\left\{\begin{array}{l}
\mathbf{Z}=\left[\begin{array}{llll}
Z_{1} & Z_{2} & \ldots & Z_{N}
\end{array}\right]^{T}, \text { and } \\
\overline{\mathbf{Z}}=\left[\begin{array}{llll}
\bar{Z}_{1} & \bar{Z}_{2} & \ldots & \bar{Z}_{N}
\end{array}\right]^{T} .
\end{array}\right.
$$

Following the assumptions that the local and nonlocal variables remain finite, and that $h_{k}$ with $k=1, \ldots, N$ are small enough, the second term in Eq. (25) driven by the nonlocal variables is negligible in comparison with the plastic dissipation given by the first term, and Eq. (25) reduces to

$$
\dot{\mathcal{D}}=\mathbf{P}:\left(\mathbf{F}^{\mathrm{e}} \cdot \dot{\mathbf{F}}^{\mathrm{p}}\right) \geq 0
$$

Using Eq. (26), one has the equality $\mathbf{P}:\left(\mathbf{F}^{\mathrm{e}} \cdot \dot{\mathbf{F}}^{\mathrm{p}}\right)=\boldsymbol{\tau}: \mathbf{D}^{\mathrm{p}}=J \boldsymbol{\sigma}: \mathbf{D}^{\mathrm{p}}$ in which $\mathbf{D}^{\mathrm{p}}$ is the 
plastic strain rate tensor given by Eq. (5). As a result, Eq. (36) becomes

$$
\dot{\mathcal{D}}=J \boldsymbol{\sigma}: \mathrm{D}^{\mathrm{p}} \geq 0
$$

The dissipation inequality can either be a priori fulfilled with suitable evolution laws of the internal variables (Peerlings et al., 2004; Forest, 2009) or can be directly accounted for in the yield criterion (Ling et al., 2018). In this work, because of the convexity of the yield surface, which is used to define the plastic flow, the condition $\boldsymbol{\sigma}: \mathbf{D}^{\mathrm{p}} \geq 0$ is always satisfied. Consequently, the dissipation inequality (37) is always satisfied. Moreover, the dissipation inequality (37) is also constrained by the balance of the plastic dissipation given by Eq. (46) in the next Section.

\section{General porous elastoplastic model}

Although the behavior of the neat matrix obeys a purely $J_{2}$ plasticity model, the behavior of the porous medium made from this matrix material exhibits a complex plastic flow behavior which generally depends on the stress invariants $I_{1}, J_{2}$ and $J_{3}$. In this section, a unified multi-mechanism nonlocal porous plasticity model is presented including the general yield surface, associative flow rule, and evolution laws for the internal variables, as well as its numerical implementation. A rate independent, isotropic elastic, and isothermal behavior is assumed. Additionally, the inelastic deformation does not affect the elastic material response.

The elastic behavior is governed by the reversible free energy part $\rho_{0} \psi^{e}$ considered in Eq. (17), written under the following form

$$
\rho_{0} \psi^{e}\left(\mathbf{E}^{\mathrm{e}}\right)=\frac{K}{2}\left[\operatorname{tr}\left(\mathbf{E}^{\mathrm{e}}\right)\right]^{2}+G \operatorname{dev}\left(\mathbf{E}^{\mathrm{e}}\right): \operatorname{dev}\left(\mathbf{E}^{\mathrm{e}}\right)
$$

where $\operatorname{tr}(\mathbf{A})$ and $\operatorname{dev}(\mathbf{A})$, respectively, represent the trace and the deviatoric parts of an arbitrary second order tensor $\mathbf{A}$ and $K$ and $G$ are the bulk and shear modulii, respectively. The corotational Kirchhoff stress $\boldsymbol{\tau}$ is then estimated from the hyperelastic potential (38) using Eq. (27) as

$$
\boldsymbol{\tau}=\rho_{0} \frac{\partial \psi^{e}}{\partial \mathbf{E}^{\mathrm{e}}}=\mathbb{C}: \mathbf{E}^{\mathrm{e}}
$$

where $\mathbb{C}=K \mathbf{I} \otimes \mathbf{I}+2 G \mathcal{I}^{\text {dev }}$ in which $\mathcal{I}^{\text {dev }}$ is the deviatoric part of the fourth order identity tensor. The first Piola-Kirchhoff stress $\mathbf{P}$ is estimated using Eq. (26) based on Eq. (39).

The plastic behavior of the porous medium depends on the geometry and distribution of voids, which are characterized by several effective parameters. In the current work, a representative cylindrical unit cell includes a void at its center, see Fig. 1(a). The geometry of such a unit cell is completely determined by several dimensionless variables: the porosity $f$, the void aspect ratio $W$, the void ligament ratio $\chi$, the void spacing ratio $\lambda$, and the $z-$ main axis (with unit vector $\mathbf{e}_{z}$ ) of the void as shown in Fig. 1. Moreover, the actual shape of the void, which might not be preserved during loading, is characterized by a shape factor, 
denoted by $\gamma$, with $\gamma=\frac{1}{2}$ for a spheroid and $\gamma=1$ for a cone (Benzerga, 2002), see Fig. 1(b). This assumes that the initial distribution of voids can be approximated by an effective periodic distribution with initial values $\chi_{0}, \lambda_{0}$, and effective initial shape $W_{0}, \gamma_{0}$. These void characteristics are related by the following relationship (Benzerga, 2002)

$$
f=\frac{V_{\text {void }}}{V_{\text {void }}+V_{\text {solid }}}=\frac{\chi^{3} W}{3 \gamma \lambda} .
$$

The last equation can be rewritten as

$$
f=\frac{f^{\text {cone }}}{\gamma}
$$

where $f^{\text {cone }}$ is the porosity of a unit cell in which a conical void with the same $\chi$ and $W$ is embedded. From Eq. (41), the parameter $\gamma$ indicates to what extent the current shape departs from the ideal conical one.

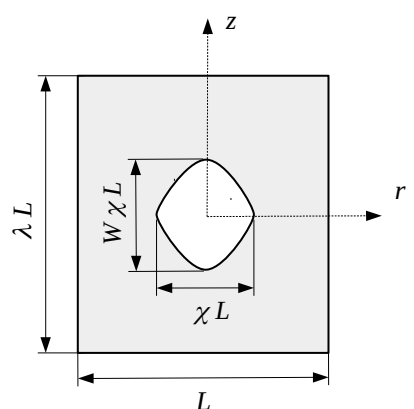

(a)

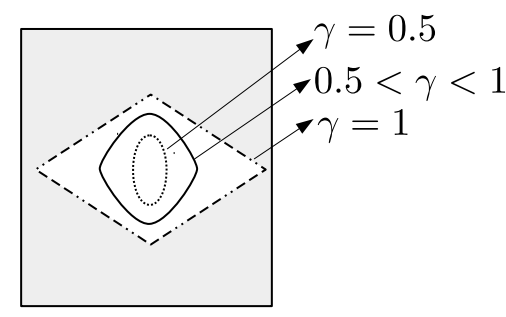

(b)

Figure 1: Cylindrical unit cell including a void at its center: (a) geometrical parameters (the void aspect ratio $W$, void ligament ratio $\chi$, and void spacing ratio $\lambda$ ) and (b) different void shapes characterized by the void shape factor $\gamma$.

When the failure mode is dominated by void growth, the porosity $f$ is a damage indicator, but when the failure mode is dominated by internal necking or shear-type coalescence, the ligament ratio $\chi$ becomes the controlling damage indicator instead of the porosity. In general, a set of parameters $\mathbf{Y}$ is used to describe the void characteristics, including the porosity and the parameters related to the shape of voids as well as its main axis $\mathbf{e}_{z}$. This vector is here given by

$$
\mathbf{Y}=\left[\begin{array}{llllll}
f & \chi & W & \lambda & \gamma & \mathbf{e}_{z}
\end{array}\right]^{T}
$$

but a more general description can be considered. Voids are assumed to be present from the beginning with initial values $\mathbf{Y}_{0}=\left[\begin{array}{llllll}f_{0} & \chi_{0} & W_{0} & \lambda_{0} & \gamma_{0} & \mathbf{E}_{z}\end{array}\right]^{T}$. 
In a general form, the porous plasticity model is summarized as follows:

$$
\begin{aligned}
& \Phi_{\mathrm{nl}}=\Phi_{\mathrm{nl}}\left(\boldsymbol{\sigma} ; \sigma_{\mathrm{Y}}, \mathbf{Y}\right) \\
& \sigma_{\mathrm{Y}}=\sigma_{\mathrm{Y}}^{0}+R\left(\varepsilon_{\mathrm{m}}\right) \\
& \mathbf{D}^{\mathrm{p}}=\dot{\mu} \mathbf{N}^{\mathrm{p}}\left(\boldsymbol{\sigma} ; \sigma_{\mathrm{Y}}, \mathbf{Y}\right) \\
& \boldsymbol{\sigma}: \mathbf{D}^{\mathrm{p}}=(1-f) \sigma_{\mathrm{Y}} \dot{\varepsilon}_{\mathrm{m}}, \\
& \dot{\mathbf{Y}}=\dot{\mathbf{Y}}(\mathbf{Y}, \overline{\mathbf{Z}}, \boldsymbol{\sigma}), \text { and } \\
& \dot{\mu} \geq 0, \Phi_{\mathrm{nl}} \leq 0, \dot{\mu} \Phi_{\mathrm{nl}}=0,
\end{aligned}
$$

where $\Phi_{\mathrm{nl}}$ is the yield function; $\sigma_{\mathrm{Y}}$ is the mean yield stress of the matrix; $\sigma_{\mathrm{Y}}^{0}$ is the initial yield stress; $R\left(\varepsilon_{\mathrm{m}}\right)$ is the strain-hardening contribution; $\varepsilon_{\mathrm{m}}$ is the mean equivalent plastic strain of the matrix; $\mu$ is the plastic multiplier; and $\mathbf{N}^{\mathrm{p}}$ is the plastic flow normal. The yield function (43) is expressed in terms of the corotational Cauchy stress tensor $\boldsymbol{\sigma}$. Moreover, the effects of the porosity and of the void shape in the yield condition should be accounted for besides strain-hardening. The use of the corotational Cauchy stress $\boldsymbol{\sigma}$ instead of the Cauchy stress $\Sigma$ is justified because the yield function is expressed in terms of the stress invariants which are identical for $\boldsymbol{\sigma}$ and $\boldsymbol{\Sigma}$, while the numerical implementation with the corotational Cauchy stress tensor can be easily performed using Eqs. (30, 39). Eq. (44) represents the isotropic hardening. Eq. (45) represents the plastic flow rule. This work considers an associative plastic flow in which $\mathrm{N}^{\mathrm{p}}$ is directly derived from $\Phi_{\mathrm{nl}}$. Eq. (46) represents the balance of the plastic dissipation in which the rate of apparent plastic work is equal to the rate of plastic work in the matrix (Gurson, 1977; Tvergaard and Needleman, 1984; Needleman and Tvergaard, 1984; Håkansson et al., 2006). The term $(1-f) \sigma_{\mathrm{Y}} \dot{\varepsilon}_{\mathrm{m}}$ represents an averaged measure of the plastic dissipation rate over the matrix material within the representative volume. The evolution of the plastic flow stress of the matrix $\sigma_{\mathrm{Y}}$ is then evaluated by postulating that it is a function of the equivalent accumulated plastic strain of the matrix $\varepsilon_{\mathrm{m}}$. Under a localized state such as during void coalescence, the use of Eq. (46) seems to underestimate the flow stress in the localization region. Despite its approximate nature, Eq. (46) was used in most studies (Benzerga et al., 2016) because it allows incorporating the isotropic hardening effect as an additional equation governing the evolution of $\sigma_{\mathrm{Y}}$. Eq. (47) describes the evolution laws for the void characteristics that depend not only on the nonlocal variables $\overline{\mathbf{Z}}$ but also on the stress state. The loading-unloading is governed by the consistency conditions (48). The explicit expressions of $\Phi_{\mathrm{nl}}$ and of the evolution laws for $\mathbf{Y}$ must be provided.

From the rate of the plastic tensor following the flow rule (45), one can define the deviatoric and volumetric equivalent plastic strains respectively by

$$
\begin{aligned}
& \dot{\varepsilon}_{\mathrm{d}}=\sqrt{\frac{2}{3} \operatorname{dev}\left(\mathbf{D}^{\mathrm{p}}\right): \operatorname{dev}\left(\mathbf{D}^{\mathrm{p}}\right)}=\dot{\mu} \sqrt{\frac{2}{3} \operatorname{dev}\left(\mathbf{N}^{\mathrm{p}}\right): \operatorname{dev}\left(\mathbf{N}^{\mathrm{p}}\right)}, \text { and } \\
& \dot{\varepsilon}_{\mathrm{v}}=\operatorname{tr}\left(\mathbf{D}^{\mathrm{p}}\right)=\dot{\mu} \operatorname{tr}\left(\mathbf{N}^{\mathrm{p}}\right) .
\end{aligned}
$$

There is no restriction on the selection of the local variables $\mathbf{Z}$ used to define the nonlocal 
form and this selection does not affect the numerical implementation developed in the next section. As stated in the introduction, this work focuses on the nonlocal enhancement of the multi-surface model. An efficient regularization is required to impose the nonlocality to the three different mechanisms respectively controlled by the volumetric equivalent plastic strain, matrix equivalent plastic strain, and the deviatoric equivalent plastic strain. The local variables and their nonlocal counterparts are chosen as

$$
\mathbf{Z}=\left[\begin{array}{lll}
\varepsilon_{\mathrm{v}} & \varepsilon_{\mathrm{m}} & \varepsilon_{\mathrm{d}}
\end{array}\right]^{T} \text { and } \overline{\mathbf{Z}}=\left[\begin{array}{lll}
\bar{\varepsilon}_{\mathrm{v}} & \bar{\varepsilon}_{\mathrm{m}} & \bar{\varepsilon}_{\mathrm{d}}
\end{array}\right]^{T} .
$$

The fully implicit integration scheme of the porous plasticity model described by Eqs. (43-48) is detailed in Appendix A. From the known kinematic inputs $\mathbf{F}, \overline{\mathbf{Z}}$ and internal variables, the unknown outputs $\mathbf{P}, \mathbf{Z}$, and their tangent operators $\frac{\partial \mathbf{P}}{\partial \mathbf{F}}, \frac{\partial \mathbf{P}}{\partial \mathbf{Z}}, \frac{\partial \mathbf{Z}}{\partial \mathbf{F}}$, and $\frac{\partial \mathbf{Z}}{\partial \overline{\mathbf{Z}}}$ have to be evaluated for an iterative finite element procedure.

\section{Multi-surface model}

In this section, the general nonlocal framework described in Section 3 is particularized into the multi-surface ductile damage model. The void growth solution corresponding to a diffused mode of the plasticity around the voids is governed by the GTN model involving the correction proposed by Nahshon and Hutchinson (2008). The localization solution corresponding to the void coalescence phase has two variants whether the internal necking mechanism or shearing mechanism is activated. The internal necking mechanism follows an heuristic extension of the Thomason condition with the use of the maximum principal stress (MPS) as driving term, leading to the MPS-based Thomason model. The void shearing coalescence mechanism is assumed to be governed by the maximum shear stress (MSS), leading to the MSS-based model. Moreover, the following assumptions on the void evolution are adopted in the present work:

- During the growth phase, voids are initially spherical $\left(W_{0}=1\right.$ and $\left.\gamma_{0}=0.5\right)$ and do not depart significantly from their initial shape (Besson, 2009). As a result, one can consider $\dot{W}=0, \dot{\gamma}=0$. This is a strong assumption as it is well known that the voids shape changes, especially in the low stress triaxiality regime. Additionally, voids can nucleate with a flat initial shape or an elongated prolate shape. The void shape effects will be partly corrected through the fitting of the $q_{2}$ parameter of the GTN yield surface. Furthermore, in a variety of metallic alloys, nucleation occurs quite late followed by early void coalescence. A very accurate treatment of the void growth phase in these materials is thus less essential. Finally, regarding non spherical initial shape, one can always define a corresponding effective spherical void. Once again, these approximations are real but could be corrected with further elaboration of this model following other works (Pardoen, 2006; Benzerga et al., 2016).

- During the coalescence phase, the void axes are attached to a coordinate system defined by the principle stress directions. Additionally, the voids are supposed to maintain a spheroidal shape. As a result, $\dot{\gamma}=0$. 
Because of these assumptions, the GTN model can be used to model the void growth phase, and the evolution of the void main axis $\mathbf{e}_{z}$ does not need to be considered. The void characteristics vector given by Eq. (42) reduces to

$$
\mathbf{Y}=\left[\begin{array}{llll}
f & \chi & W & \lambda
\end{array}\right]^{T} .
$$

Unlike $f$, which is an isotropic measure, the other void characteristics are determined with respect to the direction of the coalescence plane, see Fig. 1.

\subsection{Void growth phase: GTN model}

\subsubsection{Yield function}

The GTN yield function (Tvergaard and Needleman, 1984; Needleman and Tvergaard, 1984) can be written under the canonical form ${ }^{2}$

$$
\Phi_{\mathrm{nl}}=\Phi_{\mathrm{G}}=\frac{\hat{\sigma}_{\mathrm{G}}}{\sigma_{\mathrm{Y}}}-1
$$

where $\hat{\sigma}_{\mathrm{G}}$ denotes the GTN effective stress, which is given by

$$
\hat{\sigma}_{\mathrm{G}}\left(\sigma_{\mathrm{eq}}, p^{\prime}, \sigma_{\mathrm{Y}}, f\right)=\frac{\sqrt{\sigma_{\mathrm{eq}}^{2}+2 \sigma_{\mathrm{Y}}^{2} f q_{1}\left[\cosh \left(\frac{3}{2} q_{2} \frac{p^{\prime}}{\sigma_{\mathrm{Y}}}\right)-1\right]}}{1-q_{1} f} .
$$

In the last equation, $\sigma_{\text {eq }}=\sqrt{\frac{3}{2} \operatorname{dev}(\boldsymbol{\sigma}): \operatorname{dev}(\boldsymbol{\sigma})}$ is the von Mises equivalent stress related to the $J_{2}$ stress invariant by $\sigma_{\text {eq }}=\sqrt{3 J_{2}}, p^{\prime}=\frac{\operatorname{tr}(\boldsymbol{\sigma})}{3}$ is the pressure related to the $I_{1}$ stress invariant by $p^{\prime}=\frac{I_{1}}{3}$, and $q_{1}$ and $q_{2}$ are two material parameters. These two parameters $q_{1}$ and $q_{2}$ were introduced to account for voids interaction, matrix material hardening, and void shape effects. When $f=0$, a conventional $J_{2}$-von Mises yield surface with isotropic hardening is recovered. The other void-related parameters do not affect the void growth response. With the use of the yield surface (53), the associative plastic normal reads

$$
\mathbf{N}^{\mathrm{p}}=\mathbf{N}_{\mathrm{G}}^{\mathrm{p}}=\sigma_{\mathrm{Y}} \frac{\partial \Phi_{\mathrm{G}}}{\partial \boldsymbol{\sigma}}=\frac{\partial \hat{\sigma}_{\mathrm{G}}}{\partial \boldsymbol{\sigma}}
$$

where $\sigma_{\mathrm{Y}}$ is introduce to generate a dimensionless tensor. The expression of $\mathbf{N}_{\mathrm{G}}^{\mathrm{p}}$ is detailed in Appendix F.

\footnotetext{
${ }^{2}$ In the literature, the usual form is expressed as $\Phi_{\mathrm{G}}=\frac{\sigma_{\mathrm{eq}}^{2}}{\sigma_{\mathrm{Y}}^{2}}+2 f q_{1} \cosh \left(\frac{3}{2} q_{2} \frac{p^{\prime}}{\sigma_{\mathrm{Y}}}\right)-1-q_{1}^{2} f^{2}$.
} 


\subsubsection{Evolution laws of the void characteristics: local version}

The evolution of the porosity results from the growth of existing voids and the nucleation of new voids, and can be related to the plastic deformation with the following equation

$$
\dot{f}=\underbrace{(1-f) \operatorname{tr}\left(\mathbf{D}^{\mathrm{p}}\right)}_{\dot{f}_{\mathrm{gr}}}+\underbrace{A_{n}\left(\varepsilon_{\mathrm{m}}\right) \dot{\varepsilon}_{\mathrm{m}}}_{\dot{f}_{\mathrm{nu}}}+\underbrace{k_{\omega} \phi_{\eta} \phi_{\omega} f \frac{\operatorname{dev}(\boldsymbol{\sigma}): \mathbf{D}^{\mathrm{p}}}{\sigma_{\mathrm{eq}}}}_{\dot{f}_{\mathrm{sh}}},
$$

where the first term $\dot{f}_{\text {gr }}$ (so-called growth term) is the usual term associated to the plastic incompressibility of the matrix (Tvergaard and Needleman, 1984; Needleman and Tvergaard, 1984); the second term $\dot{f}_{\text {nu }}$ (so-called nucleation term) is the contribution of new voids nucleated due to particle debonding or cracking under the assumption of a plastic strain controlled nucleation (Chu and Needleman, 1980); and the third term $\dot{f}_{\text {sh }}$ (so-called shear term) corresponds to an effective change of porosity due to void deformation and reorientation occurring under low stress triaxiality and shear-dominated distortions (Nahshon and Hutchinson, 2008). In the nucleation term, $A_{n}$ is the nucleation intensity function, which can be taken as a function of the matrix equivalent plastic strain $\varepsilon_{\mathrm{m}}$ (Chu and Needleman, 1980; Besson et al., 2003). In the shear term, $k_{\omega}$ is a material constant (Nahshon and Hutchinson, 2008), $\phi_{\eta}=\phi_{\eta}(\eta)$, where $\eta=\frac{p^{\prime}}{\sigma_{\mathrm{eq}}}$ is the stress triaxiality, and $\phi_{\omega}=\phi_{\omega}(\omega)$, where $\omega$ is defined in terms of the second invariant $\sigma_{\text {eq }}$ and of the third invariant $J_{3}=\operatorname{det}(\operatorname{dev}(\boldsymbol{\sigma}))$ of the deviatoric part of the stress tensor as

$$
\omega=\frac{27 J_{3}}{2 \sigma_{\mathrm{eq}}{ }^{3}} .
$$

For a general loading case, one has $-1 \leq \omega \leq 1$. In particular, $\omega^{2}=1$ for axisymmetric stress states and $\omega=0$ for a combination of a pure shear stress with a hydrostatic contribution. The introduction of $k_{\omega}$ in Eq. (56) in order to capture a softening effect under shear loading is purely phenomenological so that $f$ should be considered in this version of the GTN model as a damage variable. As a matter of fact, this heuristic addition to the GTN model is a simple way to artificially account for void distortion and rotation under shear. Following Nahshon and Hutchinson (2008), $\phi_{\eta}$ is equal to 1 , and $\phi_{\omega}$ is equal to $1-\omega^{2}$. However, this choice can strongly affect the void growth under high stress triaxiality. To overcome this issue, Nielsen and Tvergaard (2010) proposed to consider the function $\phi_{\eta}$ depending on the level of stress triaxiality. At very low stress triaxiality, $\phi_{\eta}$ is close to 1 while $\phi_{\eta}$ vanishes at high stress triaxiality. In the present work, $\phi_{\eta}$ and $\phi_{\omega}$ respectively take the forms

$$
\phi_{\eta}=\exp \left[-\frac{1}{2}\left(\frac{\eta}{\eta_{s}}\right)^{2}\right], \text { and } \phi_{\omega}=1-\omega^{2},
$$

where $\eta_{s}$ is an additional parameter. The shear term almost vanishes when $\eta>3 \eta_{s}$. The use of Eq. (55) with the definition of the deviatoric and volumetric equivalent plastic strains 
following Eq. (49) leads to ${ }^{3}$

$$
\frac{\operatorname{dev}(\boldsymbol{\sigma}): \mathbf{D}^{\mathrm{p}}}{\sigma_{\mathrm{eq}}}=\frac{\operatorname{dev}(\boldsymbol{\sigma})}{\sigma_{\mathrm{eq}}}: \dot{\mu} \frac{\partial \hat{\sigma}_{\mathrm{G}}}{\partial \sigma_{\mathrm{eq}}} \frac{3 \operatorname{dev}(\boldsymbol{\sigma})}{2 \sigma_{\mathrm{eq}}}=\dot{\mu} \frac{\partial \hat{\sigma}_{\mathrm{G}}}{\partial \sigma_{\mathrm{eq}}}=\dot{\varepsilon}_{\mathrm{d}},
$$

from which, using Eq. (50), Eq. (56) can be rewritten as

$$
\dot{f}=(1-f) \dot{\varepsilon}_{\mathrm{v}}+A_{n}\left(\varepsilon_{\mathrm{m}}\right) \dot{\varepsilon}_{\mathrm{m}}+k_{\omega} \phi_{\eta} \phi_{\omega} f \dot{\varepsilon}_{\mathrm{d}} .
$$

The current void spacing ratio $\lambda$ evolves from its initial value $\lambda_{0}$ following the deformation history. Its evolution is assumed to be controlled by $\dot{\varepsilon}_{\mathrm{d}}$ through the following relationship (Benzerga, 2002; Benzerga and Leblond, 2010; Benzerga et al., 2016)

$$
\dot{\lambda}=\kappa \lambda \dot{\varepsilon}_{\mathrm{d}}
$$

where $\kappa$ is a user parameter, so-called void spacing control factor. The value of $\kappa$ depends on the distribution of voids. Following Benzerga et al. (2016), $\kappa=1.5$ for a periodic distribution under tension, $\kappa=0$ for a random distribution, and $0 \leq \kappa \leq 1.5$ for a clustered distribution of voids.

As explained at the beginning of this section, the simplified assumption of considering spherical voids is adopted during the growth phase using the GTN model to capture the underlying physics with a reduced number of parameters. As a result of the evolution laws of the porosity and of the void spacing ratio following Eqs. (60, 61), the evolution of the void ligament ratio $\chi$ is directly deduced from Eq. (40) as

$$
\chi=\left(\frac{3 f \lambda}{2}\right)^{\frac{1}{3}}
$$

Clearly, the characteristics of the spherical voids in the evolution laws $(60,61,62)$ do not correspond to the "true" values due to the rotation and distortion of real voids, but they are seen as corrected measures which are considered to obtain an equivalent constitutive behavior. Assuming $\tilde{\chi}, \tilde{\gamma}, \tilde{\lambda}, \tilde{W}$, and $\tilde{f}$ being the true values of the void characteristics, Eq. (40) can be rewritten as

$$
\tilde{\chi}^{3}=\frac{3 \tilde{\gamma} \tilde{\lambda} \tilde{f}}{\tilde{W}}
$$

\footnotetext{
${ }^{3}$ Using the equality $\frac{\partial \sigma_{\mathrm{eq}}}{\partial \boldsymbol{\sigma}}=\frac{3 \operatorname{dev}(\boldsymbol{\sigma})}{2 \sigma_{\mathrm{eq}}}$, one has $\operatorname{dev}\left(\mathbf{D}^{\mathrm{p}}\right)=\dot{\mu} \frac{\partial \hat{\sigma}_{\mathrm{G}}}{\partial \sigma_{\mathrm{eq}}} \frac{3 \operatorname{dev}(\boldsymbol{\sigma})}{2 \sigma_{\mathrm{eq}}}$ since $\hat{\sigma}_{\mathrm{G}}$ depends on $p^{\prime}$ and $\sigma_{\mathrm{eq}}$. As a result, one has $\dot{\varepsilon}_{\mathrm{d}}=\sqrt{\frac{2}{3} \operatorname{dev}\left(\mathbf{D}^{\mathrm{p}}\right): \operatorname{dev}\left(\mathbf{D}^{\mathrm{p}}\right)}=\dot{\mu} \frac{\partial \hat{\sigma}_{\mathrm{G}}}{\partial \sigma_{\mathrm{eq}}}$.
} 
Eqs. $(62,63)$ imply the following equivalence

$$
\tilde{\chi}=\left(\frac{3 \tilde{\gamma} \tilde{\lambda} \tilde{f}}{\tilde{W}}\right)^{\frac{1}{3}} \Leftrightarrow \chi=\left(\frac{3 \lambda f}{2}\right)^{\frac{1}{3}}
$$

where $\chi, \lambda$, and $f$ are the "equivalent" void characteristics considered in the present model in the context of the simplified growth assumption. The evolution laws of the void characteristics following Eqs. $(60,61)$ are expected to capture the equivalence $(64)$. Consequently, this equivalence requires the following parameters to be evaluated: $q_{1}$ and $q_{2}$ (for the contribution of the volumetric plastic deformation), $k_{\omega}$ and $\eta_{s}$ (for the contribution of the deviatoric plastic deformation ), and $\kappa$ (for the evolution of the void spacing ratio), e.g. either from direct identification on experimental tests or from unit cell simulations as shown in Section 5.4. The void nucleation can be accounted for but the equivalence (64) is still assumed. Although the parameter $k_{\omega}$ was introduced in the GTN model by Nahshon and Hutchinson (2008) as an enhanced damage growth parameter in order to account for shear failure, $k_{\omega}$ is seen here as a correcting factor of the void evolution during the growth phase introduced in order to satisfy the equivalence (64). The failure under shear-dominated loading conditions is captured using the shear driven coalescence model as detailed in Section 4.3.

In summary, the evolution laws for $f, W$, and $\lambda$ in their local forms are given by

$$
\begin{cases}\dot{f} & =(1-f) \dot{\varepsilon}_{\mathrm{v}}+A_{n}\left(\varepsilon_{\mathrm{m}}\right) \dot{\varepsilon}_{\mathrm{m}}+k_{\omega} \phi_{\eta} \phi_{\omega} f \dot{\varepsilon}_{\mathrm{d}} \\ \dot{W} & =0, \text { and } \\ \dot{\lambda} & =\kappa \lambda \dot{\varepsilon}_{\mathrm{d}} .\end{cases}
$$

The void ligament ratio $\chi$ is estimated using Eq. (62).

\subsubsection{Evolution laws of the void characteristics: nonlocal enhancement}

As shown in Eq. (65), the expansion of the voids is governed by three different terms which are driven by the volumetric equivalent plastic strain $\dot{\varepsilon}_{\mathrm{v}}$, the deviatoric equivalent plastic strain $\dot{\varepsilon}_{\mathrm{d}}$, and the matrix equivalent plastic strain $\dot{\varepsilon}_{\mathrm{m}}$. In order to avoid the loss of solution uniqueness for any kind of failure mechanism, the dependency of the evolution laws on the local variables $\varepsilon_{\mathrm{v}}, \varepsilon_{\mathrm{d}}$, and $\varepsilon_{\mathrm{m}}$ in Eq. (65) is then modified using their nonlocal counterparts $\bar{\varepsilon}_{\mathrm{v}}, \bar{\varepsilon}_{\mathrm{d}}$, and $\bar{\varepsilon}_{\mathrm{m}}$, leading to

$$
\begin{cases}\dot{f} & =(1-f) \dot{\bar{\varepsilon}}_{\mathrm{v}}+A_{n}\left(\bar{\varepsilon}_{\mathrm{m}}\right) \dot{\bar{\varepsilon}}_{\mathrm{m}}+k_{\omega} \phi_{\eta} \phi_{\omega} f \dot{\bar{\varepsilon}}_{\mathrm{d}} \\ \dot{W} & =0, \text { and } \\ \dot{\lambda} & =\kappa \lambda \dot{\bar{\varepsilon}}_{\mathrm{d}} .\end{cases}
$$

The evolution of $\chi$ follows Eq. (62). 


\subsection{Void coalescence by internal necking: MPS-based Thomason model}

\subsubsection{Onset of internal necking}

At the onset of void coalescence, plastic deformation starts localizing inside the ligament between voids while the material outside of the ligament zone unloads elastically. This regime of plastic flow involves velocity fields completely different than during the diffuse growth stage and which are very different than the ones used to generate the Gurson resolution. According to Thomason (Thomason, 1985a,b), based on an appropriate choice of the velocity fields, the onset of coalescence occurs when the maximum axial stress $\sigma_{z z}$ supported by the top surface of the localization zone reaches a limit load defined by $C_{\mathrm{Tf}} \sigma_{\mathrm{Y}}$, where $\sigma_{\mathrm{Y}}$ is the yield stress inside the localization zone and $C_{\mathrm{Tf}}$ is the limit-load constraint factor whose value depends on the considered microstructure (see the recent contribution by Benzerga and Leblond (2014) for a reassessment of the Thomason analysis). An empirical fit of the numerical solution of the minimization problems by Thomason $(1985 \mathrm{a}, \mathrm{b})$ leads to the following condition of the onset of coalescence

$$
\sigma_{z z}-\sigma_{\mathrm{Y}} C_{\mathrm{Tf}}=0
$$

where $C_{\mathrm{Tf}}$ is a function of the void ligament ratio $\chi$ and of the void aspect ratio $W$ as

$$
C_{\mathrm{Tf}}(W, \chi)=\left(1-\chi^{2}\right)\left[h\left(\frac{1-\chi}{W \chi}\right)^{2}+g \sqrt{\frac{1}{\chi}}\right],
$$

where $h$ and $g$ are two constants. Although in the original model $h=0.1$ and $g=1.2$ are identified, following Pardoen and Hutchinson (2000), the value of $h$ should be calibrated as a function of the strain hardening exponent of the matrix, and $g=1.24$.

Although first derived for axisymmetric configurations, see Fig. 1, the Thomason condition (67) is herein heuristically extended for any stress state. Under an axisymmetric stress state $\sigma_{z z}>\sigma_{r r}$, one has $\sigma_{z z}=p^{\prime}+\frac{2}{3} \sigma_{\text {eq }}$, from which Eq. (67) can be rewritten as

$$
p^{\prime}+\frac{2}{3} \sigma_{\mathrm{eq}}-\sigma_{\mathrm{Y}} C_{\mathrm{Tf}}=0 .
$$

Since Eq. (69) is expressed in terms of the stress invariants $\left(p^{\prime}\right.$ and $\sigma_{\text {eq }}$ ), Eq. (69) was often adopted to detect the onset of coalescence as well as to govern the coalescence phase in general loading conditions (Benzerga et al., 2002; Besson, 2009; Benzerga and Leblond, 2010; Pardoen and Hutchinson, 2000, 2003; Pardoen, 2006). However, rigorously speaking, the extended form (69) of the condition (67) for general stress states is valid only under axisymmetric loading conditions. The effect of the Lode parameter on the onset of coalescence is not accounted for. In this work, a new extension of the condition (67) is proposed as

$$
\max \operatorname{eig}(\boldsymbol{\sigma})-C_{\mathrm{Tf}} \sigma_{\mathrm{Y}}=0
$$

where max eig $(\boldsymbol{\sigma})$ denotes the maximum principal stress of $\boldsymbol{\sigma}$. We however note that this extension does not intend to capture failure under a shear void coalescence mode; for such 
failure mechanisms a shear-driven yield function will be introduced in Section 4.3. Clearly Eq. (70) reduces to Eq. (69) under axisymmetric loading conditions. In general loading conditions, three eigenvalues $\sigma_{1} \geq \sigma_{2} \geq \sigma_{3}$ of the stress tensor $\boldsymbol{\sigma}$ are given as a function of the stress invariants as

$$
\left[\begin{array}{l}
\sigma_{1} \\
\sigma_{2} \\
\sigma_{3}
\end{array}\right]=p^{\prime}\left[\begin{array}{l}
1 \\
1 \\
1
\end{array}\right]+\frac{2}{3} \sigma_{\text {eq }}\left[\begin{array}{c}
\cos \theta \\
\cos \left(\frac{2 \pi}{3}-\theta\right) \\
\cos \left(\frac{2 \pi}{3}+\theta\right)
\end{array}\right]
$$

where $\theta \in\left[0 \frac{\pi}{3}\right]$ is the Lode angle which is estimated from the second and the third invariants of $\operatorname{dev}(\boldsymbol{\sigma})$ as

$$
\theta\left(\sigma_{\text {eq }}, J_{3}\right)=\frac{1}{3} \arccos \omega
$$

with $\omega=\omega\left(\sigma_{\text {eq }}, J_{3}\right)$ given by Eq. (57). The Lode angle $\theta$ relates directly to the Lode parameter $L$ through the following relationship

$$
L=\frac{2 \sigma_{2}-\sigma_{1}-\sigma_{3}}{\sigma_{1}-\sigma_{3}}=\sqrt{3} \tan \left(\theta-\frac{\pi}{6}\right) .
$$

Combining Eqs. $(70,71)$ leads to the following Lode-sensitive coalescence criterion

$$
p^{\prime}+\frac{2}{3} \sigma_{\text {eq }} \cos \theta-\sigma_{\mathrm{Y}} C_{\mathrm{Tf}}=0
$$

Since the maximum principal stress $\sigma_{1}=p^{\prime}+\frac{2}{3} \sigma_{\text {eq }} \cos \theta$ is a convex function of the stress tensor $\boldsymbol{\sigma}$, see Appendix G for proof, Eq. (74) is considered as the yield condition governing the internal necking process.

\subsubsection{MPS-based Thomason yield function}

During the void coalescence phase, following the assumption that the plasticity inside the void ligament remains in a limit load state (Benzerga et al., 2002), the condition (74) for the onset of void coalescence can be used as a Lode and triaxiality sensitive yield condition (so-called MPS-based Thomason yield surface) governing the coalescence process by internal necking, leading to

$$
\Phi_{\mathrm{nl}}=\Phi_{\mathrm{T}}=\frac{\hat{\sigma}_{\mathrm{T}}}{\sigma_{\mathrm{Y}}}-1
$$

where $\hat{\sigma}_{\mathrm{T}}$ denotes the Thomason effective stress, which is given by

$$
\hat{\sigma}_{\mathrm{T}}=\frac{1}{C_{\mathrm{Tf}}}\left(\frac{2}{3} \sigma_{\mathrm{eq}} \cos \theta+\left|p^{\prime}\right|\right)
$$


In the last equation, $\left|p^{\prime}\right|$ is considered in order to generate a symmetric equation under negative pressure as proposed by Pardoen and Hutchinson (2003).

With the use of the Thomason effective stress following Eq. (76), the MPS-based Thomason yield surface (75) has singularities at $p^{\prime}=0$ (under pure shear), at $\sigma_{\text {eq }}=0$ (under hydrostatic pressure), and at $\theta=\frac{\pi}{3}$ (at the stress state $\sigma_{1}=\sigma_{2}>\sigma_{3}$ ). These corners are rounded in order to produce a smooth normal vector all along the yield surface as detailed in Appendix E. The associative plastic normal is given by

$$
\mathbf{N}^{\mathrm{p}}=\mathbf{N}_{\mathrm{T}}^{\mathrm{p}}=\sigma_{\mathrm{Y}} \frac{\partial \Phi_{\mathrm{T}}}{\partial \boldsymbol{\sigma}}=\frac{\partial \hat{\sigma}_{\mathrm{T}}}{\partial \boldsymbol{\sigma}}
$$

where $\sigma_{\mathrm{Y}}$ is introduced to obtain a dimensionless tensor. The expression of $\mathbf{N}_{\mathrm{T}}^{\mathrm{p}}$ is given in Appendix F.

4.2.3. Evolution laws of the void characteristics in the internal necking phase: local version

During the coalescence phase, the void expansion no longer follows Eq. (60). The evolution laws for $\chi, W$, and $\lambda$ are deduced from the plastic incompressibility of the matrix following the work of Benzerga (2002) as

$$
\left\{\begin{array}{l}
\dot{\chi}=\frac{3}{4} \frac{\lambda}{W}\left(\frac{3}{2 \chi^{2}}-1\right) \dot{\varepsilon}_{\mathrm{d}}, \\
\dot{W}=\frac{9}{4} \frac{\lambda}{\chi}\left(1-\frac{1}{2 \chi^{2}}\right) \dot{\varepsilon}_{\mathrm{d}}, \text { and } \\
\dot{\lambda}=\kappa \lambda \dot{\varepsilon}_{\mathrm{d}}
\end{array}\right.
$$

The porosity $f$ is directly estimated using Eq. (40). Note that the void ligament ratio $\chi$ can be considered as the controlling damage indicator during void coalescence.

\subsubsection{Evolution laws of the void characteristics in the internal necking phase: nonlocal enhancement}

As shown in Eq. (78), the evolution of $\chi$ is governed by the deviatoric equivalent plastic strain $\dot{\varepsilon}_{\mathrm{d}}$. Since the MPS-based Thomason model is used only after the onset of coalescence, the nonlocal regulation of this model must follow the nonlocal regulation of the GTN model. Using the nonlocal variables that have been selected in Eq. (51), a fully nonlocal formulation is proposed as

$$
\left\{\begin{array}{l}
\dot{\chi}=\frac{3}{4} \frac{\lambda}{W}\left(\frac{3}{2 \chi^{2}}-1\right) \dot{\bar{\varepsilon}}_{\mathrm{d}}, \\
\dot{W}=\frac{9}{4} \frac{\lambda}{\chi}\left(1-\frac{1}{2 \chi^{2}}\right) \dot{\bar{\varepsilon}}_{\mathrm{d}}, \text { and } \\
\dot{\lambda}=\kappa \lambda \dot{\bar{\varepsilon}}_{\mathrm{d}}
\end{array}\right.
$$

while the porosity is still estimated using Eq. (40). 


\subsection{Void coalescence under shear: MSS-based model}

\subsubsection{Onset of the shear driven coalescence failure}

From the three eigenvalues of the stress tensor in Eq. (71), the maximum shear stress (MSS) is given by

$$
\tau_{\max }=\frac{\sigma_{1}-\sigma_{3}}{2}=\frac{\sigma_{\mathrm{eq}}}{\sqrt{3}} \sin \left(\theta+\frac{\pi}{3}\right)
$$

Following the works of Torki et al. (2015, 2017), the onset of a shear driven coalescence mode under pure shear is obtained by applying a simple rule of mixture between the yield stress in the matrix and the void. As a result, this condition reads

$$
\tau_{\max }-\left(1-\chi^{2}\right) \frac{\sigma_{\mathrm{Y}}}{\sqrt{3}}=0,
$$

where $\frac{\sigma_{\mathrm{Y}}}{\sqrt{3}}$ represent the shear flow stress of the matrix and $\chi$ is the void ligament parameter.

Combining Eqs. $(80,81)$, the onset of shear driven void coalescence can be rewritten as

$$
\sigma_{\text {eq }} \sin \left(\theta+\frac{\pi}{3}\right)-C_{\mathrm{Sf}} \sigma_{\mathrm{Y}}=0,
$$

where $C_{\mathrm{Sf}}$ denotes the shear load factor given by

$$
C_{\mathrm{Sf}}=\xi\left(1-\chi^{2}\right)
$$

In the last equation, $\xi$ is introduced in order to better capture the onset of failure in shear. The original form (Torki et al., 2015, 2017) corresponds to $\xi=1$. Since the maximum shear stress $\tau_{\text {max }}$ is a convex function of the stress tensor $\boldsymbol{\sigma}$, see Appendix G for proof, Eq. (82) is considered as the yield condition governing the shear driven coalescence process.

\subsubsection{MSS-based yield function}

The condition (82) allows defining the yield condition (so-called MSS-based yield surface) governing the shear coalescence process as

$$
\Phi_{\mathrm{nl}}=\Phi_{\mathrm{S}}=\frac{\hat{\sigma}_{\mathrm{S}}}{\sigma_{\mathrm{Y}}}-1
$$

where $\hat{\sigma}_{\mathrm{S}}$ denotes the shear effective stress, which is given as

$$
\hat{\sigma}_{\mathrm{S}}=\frac{\sigma_{\mathrm{eq}}}{C_{\mathrm{Sf}}}\left(\frac{\sin \theta}{2}+\frac{\sqrt{3} \cos \theta}{2}\right) .
$$

With the use of the shear effective stress in Eq. (85), the MSS-based yield condition (84) has singularities at $\theta=0$ and $\theta=\frac{\pi}{3}$ respectively corresponding to $\omega=1$ and $\omega=-1$. These corners are rounded in order to produce a smooth normal vector all along the yield 
surface as detailed in Appendix E. The associative plastic normal reads

$$
\mathbf{N}^{\mathrm{p}}=\mathbf{N}_{\mathrm{S}}^{\mathrm{p}}=\sigma_{\mathrm{Y}} \frac{\partial \Phi_{\mathrm{S}}}{\partial \boldsymbol{\sigma}}=\frac{\partial \hat{\sigma}_{\mathrm{S}}}{\partial \boldsymbol{\sigma}}
$$

where $\sigma_{\mathrm{Y}}$ is introduced to obtain a dimensionless equation. The expression of $\mathbf{N}_{\mathrm{S}}^{\mathrm{p}}$ is detailed in Appendix F.

\subsubsection{Evolution laws of the void characteristics in the shear-driven coalescence phase: local version}

The evolution laws for void characteristics under shear loading are available in the literature, see e.g Torki and Benzerga (2018). However, the complex description of voids requires many parameters with complex evolution laws. In the present work, phenomenological laws of the void characteristics are proposed as

$$
\left\{\begin{array}{l}
\dot{\chi}=K_{\chi} \dot{\varepsilon}_{\mathrm{d}}, \text { and } \\
\dot{W}=0,
\end{array}\right.
$$

where $K_{\chi}$ is the accelerated factor of the void ligament under the shear driven coalescence mode. As the MSS-based yield surface is insensitive to pressure, one can assume $\dot{f}=0$ and the void spacing ratio $\lambda$ can be computed using Eq. (40) by considering $\gamma=0.5$. Since only $\chi$ affects the material response during a shear driven coalescence deformation mode, the evolution laws of $W, \lambda$, and $\gamma$ must not be considered. More physical-based evolution laws will be elaborated in future works.

4.3.4. Evolution laws of the void characteristics in the shear-driven coalescence phase: nonlocal enhancement

Using the nonlocal variables that have been selected in Eq. (51), the nonlocal enhancement of the evolution laws given in Eqs. (87) follows

$$
\left\{\begin{array}{l}
\dot{f}=0, \\
\dot{\chi}=K_{\chi} \dot{\bar{\varepsilon}}_{\mathrm{d}}, \text { and } \\
\dot{W}=0 .
\end{array}\right.
$$

The void spacing ratio $\lambda$ is estimated using Eq. (40) by considering $\gamma=0.5$.

\subsection{Competition between the different plastic flow solutions}

The competition between the three modes of porosity evolution by diffuse void growth, internal necking coalescence, and shear driven void coalescence respectively governed by the GTN, the MPS-based Thomason, and MSS-based yield functions is modeled by defining an effective yield function $\Phi_{\mathrm{e}}$ as

$$
\Phi_{\mathrm{nl}}=\Phi_{\mathrm{e}}=\max \left(\Phi_{\mathrm{G}}, \Phi_{\mathrm{T}}, \Phi_{\mathrm{S}}\right)
$$


which is delimited by all three yield surfaces $\Phi_{\mathrm{G}}, \Phi_{\mathrm{T}}$, and $\Phi_{\mathrm{S}}$ defined by Eqs. $(53,75,84)$. Eq. (89) can be rewritten under the canonical form

$$
\Phi_{\mathrm{e}}=\frac{\hat{\sigma}}{\sigma_{\mathrm{Y}}}-1
$$

where $\hat{\sigma}$ denotes the effective stress, which is estimated from $\hat{\sigma}_{\mathrm{G}}, \hat{\sigma}_{\mathrm{T}}$, and $\hat{\sigma}_{\mathrm{S}}$ given by Eqs. $(54,76,85)^{4}$ as

$$
\hat{\sigma}=\max \left(\hat{\sigma}_{\mathrm{G}}, \hat{\sigma}_{\mathrm{T}}, \hat{\sigma}_{\mathrm{S}}\right) \text {. }
$$

In general, the effective yield surface (90) in combination with Eq. (91) involves singularities at the intersections between $\Phi_{\mathrm{G}}, \Phi_{\mathrm{T}}$, and $\Phi_{\mathrm{S}}$. These singularities require special treatment as the associative plastic normal is discontinuous across them. In order to resolve such singularities, a unified treatment has been proposed with a smooth transition between growth and coalescence, see the works by Morin et al. (2016); Torki (2019). The present work follows an efficient treatment based on the approximation of the infinite norm, in which Eq. (91) is replaced by its smoothed version

$$
\hat{\sigma}=\left(\hat{\sigma}_{\mathrm{G}}^{m_{\mathrm{inf}}}+\hat{\sigma}_{\mathrm{T}}^{m_{\mathrm{inf}}}+\hat{\sigma}_{\mathrm{S}}^{m_{\mathrm{inf}}}\right)^{\frac{1}{m_{\mathrm{inf}}}} .
$$

In the last equation, $m_{\text {inf }}$ is a user parameter whose value should be chosen mush larger than 1 in order to obtain an approximated version (92) close to the original one (91). When $m_{\text {inf }} \rightarrow+\infty$, the original form (91) is recovered. In this work, $m_{\text {inf }}=50$ is used. The yield surface (90) in combination with the effective stress in Eq. (92) does not exhibit any singularities, therefore the general framework described in Section 3 can be directly applied to integrate the constitutive model. With the use of the yield surface (92), the associative plastic normal reads

$$
\mathbf{N}^{\mathrm{p}}=\mathbf{N}_{\mathrm{e}}^{\mathrm{p}}=\sigma_{\mathrm{Y}} \frac{\partial \Phi_{\mathrm{e}}}{\partial \boldsymbol{\sigma}}=\frac{\partial \hat{\sigma}}{\partial \boldsymbol{\sigma}},
$$

where $\sigma_{\mathrm{Y}}$ is used to obtain a dimensionless tensor. Using Eq. (92), Eq. (93) is rewritten as

$$
\mathbf{N}_{\mathrm{e}}^{\mathrm{p}}=\frac{\partial \hat{\sigma}}{\partial \hat{\sigma}_{\mathrm{G}}} \mathbf{N}_{\mathrm{G}}^{\mathrm{p}}+\frac{\partial \hat{\sigma}}{\partial \hat{\sigma}_{\mathrm{T}}} \mathbf{N}_{\mathrm{T}}^{\mathrm{p}}+\frac{\partial \hat{\sigma}}{\partial \hat{\sigma}_{\mathrm{S}}} \mathbf{N}_{\mathrm{S}}^{\mathrm{p}}
$$

where

$$
\frac{\partial \hat{\sigma}}{\partial \hat{\sigma}_{\mathrm{G}}}=\left(\frac{\hat{\sigma}_{\mathrm{G}}}{\hat{\sigma}}\right)^{m_{\mathrm{inf}}-1}, \frac{\partial \hat{\sigma}}{\partial \hat{\sigma}_{\mathrm{T}}}=\left(\frac{\hat{\sigma}_{\mathrm{T}}}{\hat{\sigma}}\right)^{m_{\mathrm{inf}-1}}, \text { and } \frac{\partial \hat{\sigma}}{\partial \hat{\sigma}_{\mathrm{S}}}=\left(\frac{\hat{\sigma}_{\mathrm{S}}}{\hat{\sigma}}\right)^{m_{\mathrm{inf}}-1}
$$

The competition between the different damage regimes is illustrated in Fig. 2. At the

\footnotetext{
${ }^{4}$ When using the smoothing procedure on the different effective stresses as described in Appendix E, this set of equations is substituted by the set of Eqs. (54, E.3, E.5)
} 


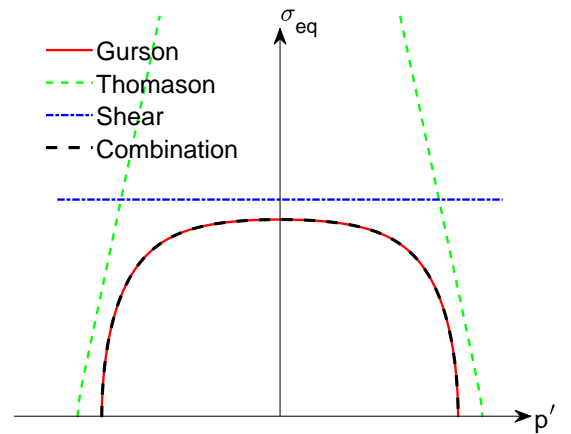

(a)

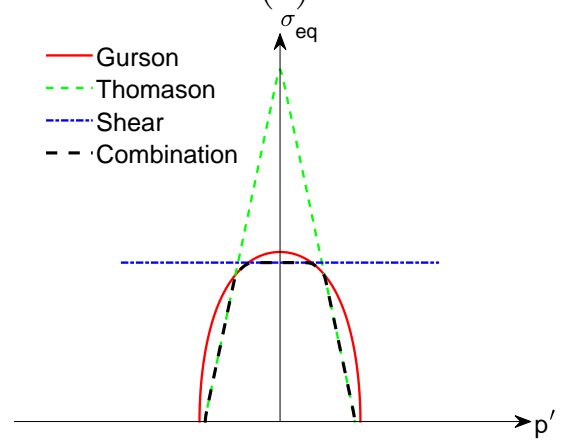

(c)

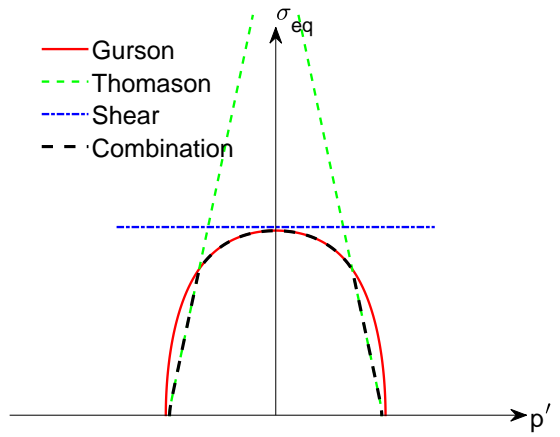

(b)

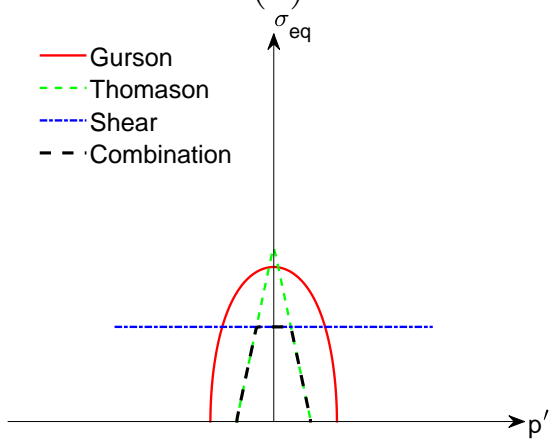

(d)

Figure 2: Combination of the GTN, MPS-based Thomason, and MSS-based yield surfaces at a constant Lode parameter: the plastic problem is governed (a) by only the GTN surface (b) by the GTN and the MPSbased Thomason yield surfaces, (c) by all the three yield surfaces, and (d) by the MPS-based Thomason and the MSS-based yield surfaces. 
beginning of the deformation, the porosity and void ligament ratio are relatively small, hence the diffuse plasticity mode governed by the GTN yield surface is preferred, see Fig. 2(a). Once the onset of (necking or shearing) coalescence is reached for a given loading path, the transition from the void growth phase to the void coalescence phase occurs. Beyond the onset of coalescence, the plastic solution is governed by the GTN yield surface coupled either with one surface of the two coalescence modes, see Fig. 2(b) or with the two coalescence modes, see Fig. 2(c). When the damage is large enough, the plastic solution is only governed by the two coalescence yield surfaces until the zero stress state is reached, see Fig. 2(d).

Since the evolution from one plastic mode to another plastic mode has been smoothed with the yield surface $(92)$, the conditions $(74,82)$ should not be used to detect the onset of coalescence. Therefore it is important to define a suitable criterion of the onset of coalescence after which the evolution laws of void characteristics switch from the ones of the growth phase following Eqs. $(65,66)$ to the ones of the internal necking phase following Eqs. $(78,79)$ or the ones of the shear driven coalescence phase following Eqs. $(87,88)$. In this work, the onset of coalescence is detected as follows:

- onset of void necking coalescence:

$$
\dot{\varepsilon}_{\mathrm{m}}>0, \text { and } \hat{\sigma}_{T}>\max \left(\hat{\sigma}_{G}, \hat{\sigma}_{S}\right) \text {. }
$$

- onset of void shear coalescence:

$$
\dot{\varepsilon}_{\mathrm{m}}>0, \text { and } \hat{\sigma}_{S}>\max \left(\hat{\sigma}_{G}, \hat{\sigma}_{T}\right)
$$

Additionally the present treatment assumes that the void coalescence phase cannot switch from one mode to another. Therefore, the evolution laws for the void characteristics during coalescence follow the ones of the coalescence mode whose onset condition is first met in the loading history.

The numerical implementation of the present multi-surface model is detailed in Appendix I.

\subsection{Summary of the constitutive parameters}

The material parameters of the thermomechanical nonlocal ductile fracture model are summarized in Tab. 1. These parameters can be divided into different categories ${ }^{5}$ :

(i) Elastoplastic parameters $\left(K, G, \sigma_{\mathrm{Y}}^{0}, R\right)$;

(ii) Initial parameters of void characteristics $\left(f_{0}, \chi_{0}, \lambda_{0}\right)$;

(iii) Porous-plasticity parameters $\left(q_{1}, q_{2}, A_{n}, k_{\omega}, \eta_{s}, \kappa, h, g, \xi\right.$, and $\left.K_{\chi}\right)$;

(iv) Nonlocal parameters $\left(l_{\varepsilon_{\mathrm{v}}}, l_{\varepsilon_{\mathrm{d}}}\right.$, and $\left.l_{\varepsilon_{\mathrm{m}}}\right)$.

\footnotetext{
${ }^{5}$ The regularization parameters used for smoothing the singularities of the yield surfaces are not mentioned here as they are chosen large enough to minimize their effect on the material response.
} 
Table 1: Material parameters of the nonlocal multi-surface model

\begin{tabular}{|c|c|l|}
\hline Parameter & Unit (SI) & Meaning \\
\hline$K$ & $\mathrm{~Pa}$ & Bulk modulus (see Eq. (38)) \\
\hline$G$ & $\mathrm{~Pa}$ & Shear modulus (see Eq. (38)) \\
\hline$\sigma_{\mathrm{Y}}^{0}$ & $\mathrm{~Pa}$ & Initial yield stress (see Eq. (44)) \\
\hline$R$ & $\mathrm{~Pa}$ & Strain hardening stress (see Eq. (44)) \\
\hline$f_{0}$ & - & Initial porosity \\
\hline$\chi_{0}, \lambda_{0}$ & - & Initial characteristics of voids \\
\hline$q_{1}, q_{2}$ & - & GTN coefficients (see Eq. (53)) \\
\hline$A_{n}$ & - & Nucleation intensity function (see Eq. (65, 66))) \\
\hline$k_{\omega}$ & - & Shear-controlled void growth factor (see Eqs. (65, 66)) \\
\hline$\eta_{s}$ & - & $\eta$-limited shear-controlled void growth (see Eq. (58)) \\
\hline$\kappa$ & - & Void spacing control factor (see Eq. (61)) \\
\hline$h, g$ & - & $C_{\mathrm{Tf}}$ coefficients (see Eq. (68)) \\
\hline$\xi$ & - & $C_{\text {Sf coefficient (see Eq. (83)) }}$ \\
\hline$K_{\chi}$ & - & Ligament growth rate under shear (see Eq. (87)) \\
\hline$l_{\varepsilon_{\mathrm{v}}}, l_{\varepsilon_{\mathrm{d}}}, l_{\varepsilon_{\mathrm{m}}}$ & $\mathrm{m}$ & Nonlocal lengths (see Eqs. (19, 32)) \\
\hline \multicolumn{2}{|l}{}
\end{tabular}

In general, the identification of a parameter of such a material model can be performed either by direct experimental characterization (Pardoen, 2006), by inverse modeling based on mechanical tests (Xue et al., 2010; Dunand and Mohr, 2011) depending on its physical meaning, or by using, for some of them, finite element unit cell calculations.

\section{Loading path dependence on the onset of coalescence}

In this section, we show that the proposed multi-surface model can capture the variations of failure scenarios under different loading conditions. To this end, the void growth phase is considered under proportional loading conditions, i.e characterized by a constant stress triaxiality $\eta=\frac{p^{\prime}}{\sigma_{\text {eq }}}$ and a constant Lode parameter $L$, which directly relates to the Lode angle $\theta$, until reaching the onset of void coalescence.

\subsection{Analytical void growth solution under proportional loading conditions}

During the diffuse void growth phase, Eqs. $(43,45,48)$ can be rewritten in the particular case of the GTN yield surface (53) respectively as follows

$$
\begin{aligned}
& X^{2}+2 q_{1} f \cosh \left(1.5 q_{2} \eta X\right)-q_{1}^{2} f^{2}-1=0, \\
& \dot{\varepsilon}_{\mathrm{v}}=\phi \dot{\varepsilon}_{\mathrm{d}}, \text { and } \\
& X \dot{\varepsilon}_{\mathrm{d}}+\eta X \dot{\varepsilon}_{\mathrm{v}}=(1-f) \dot{\varepsilon}_{\mathrm{m}}
\end{aligned}
$$

where $X=\frac{\sigma_{\text {eq }}}{\sigma_{\mathrm{Y}}}$, and

$$
\phi(f, X)=\frac{3 q_{1} q_{2} f \sinh \left(1.5 q_{2} \eta X\right)}{28^{2 X}} .
$$


In the absence of void nucleation, Eq. (65) can be rewritten as

$$
\dot{f}=(1-f) \dot{\varepsilon}_{\mathrm{v}}+k_{\omega} \phi_{\eta} \phi_{\omega} f \dot{\varepsilon}_{\mathrm{d}} .
$$

The evolution laws for the other void characteristics following Eqs. (61, 62) can be integrated, leading to

$$
\lambda=\lambda_{0} \exp \left(\kappa \varepsilon_{\mathrm{d}}\right) \text { and } \chi=\left(\frac{3 f \lambda_{0}}{2}\right)^{\frac{1}{3}} \exp \left(\frac{\kappa \varepsilon_{\mathrm{d}}}{3}\right)
$$

The onset of the necking mode of coalescence is detected using Eq. (74) as

$$
X\left(\eta+\frac{2}{3} \cos \theta\right)-C_{\mathrm{Tf}}=0
$$

where $C_{\mathrm{Tf}}$ is estimated by Eq. (68) using $h=0.1$ and $g=1.24$. The onset of the shear mode of coalescence is verified following Eq. (82) as

$$
X \sin \left(\theta+\frac{\pi}{3}\right)-C_{\mathrm{Sf}}=0,
$$

where $C_{\mathrm{Sf}}$ is estimated using Eq. (83). The last equation shows that the onset of shear coalescence predicted by the multi-surface model is insensitive to the sign of the Lode parameter contrarily to the onset of internal necking coalescence predicted by Eq. (104).

Under a constant stress triaxiality $(\eta)$ and a constant Lode parameter $(L)$, the void growth phase dictated by Eqs. (98 - 103) can be solved until the onset of coalescence defined by Eqs. $(104,105)$ is met in order to find the solutions of $X, \varepsilon_{\mathrm{v}}, \varepsilon_{\mathrm{d}}, f, \lambda$, and $\chi$ as a function of the plastic dissipation driven by $\varepsilon_{\mathrm{m}}$ without the need of an explicit form of the hardening law of the matrix described by Eq. (44). For this purpose, Eqs. (99, 100, 102) can be rewritten as

$$
\left[\begin{array}{c}
\dot{f} \\
\dot{\varepsilon}_{\mathrm{v}} \\
\dot{\varepsilon}_{\mathrm{d}}
\end{array}\right]=\frac{1-f}{X(1+\eta \phi)}\left[\begin{array}{c}
(1-f) \phi+k_{\omega} \phi_{\eta} \phi_{\omega} f \\
\phi \\
1
\end{array}\right] \dot{\varepsilon}_{\mathrm{m}} .
$$

The last equation is solved using an explicit Euler scheme from the initial solution $\left(f=f_{0}\right.$, $\varepsilon_{\mathrm{v}}=0, \varepsilon_{\mathrm{d}}=0, \varepsilon_{\mathrm{m}}=0$, and $X=X_{0}$ ), in which $X_{0}$ is obtained by solving Eq. (98) with $f=f_{0}$. From these solutions, the other void characteristics are known from using Eqs. (103), and the value of $X$ is found by solving the nonlinear equation (98) once $f$ is known. The onset of coalescence is detected by Eqs. (104, 105). In the following, the ductility defined by the deviatoric equivalent plastic strain at the onset of coalescence (denoted by $\left.\varepsilon_{\mathrm{d} c}\right)$ predicted by the current multi-surface model is investigated under different constant Lode parameters and under different constant stress triaxialities. 


\subsection{Failure under pure shear}

The parameter $\xi$ considered in Eq. (83) plays an important role in the initiation of the failure under shear loading. In order to better understand its contribution, a pure shear condition (characterized by $\eta=0$ and $L=0$ ) is first studied. For this purpose, Eq. (102) is easily integrated since $\phi_{\eta}=1$ when $\eta=0$ and $\phi_{\omega}=1$ when $L=0$, yielding

$$
f=f_{0} \exp \left(k_{\omega} \varepsilon_{\mathrm{d}}\right) \text {. }
$$

The GTN yield surface (98) under a pure shear condition reduces to

$$
X=1-q_{1} f=1-q_{1} f_{0} \exp \left(k_{\omega} \varepsilon_{\mathrm{d}}\right) .
$$

The energy balance (100) becomes

$$
\frac{1-q_{1} f}{1-f} \dot{\varepsilon}_{\mathrm{d}}=\dot{\varepsilon}_{\mathrm{m}}
$$

Shear coalescence is the preferred failure mode under pure shear loading and the onset condition following Eq. (105) reads

$$
X=C_{\mathrm{Sf}}(\chi)=\xi\left\{1-\chi_{0}^{2} \exp \left[\frac{2}{3}\left(\kappa+k_{\omega}\right) \varepsilon_{\mathrm{d} s}\right]\right\},
$$

where the function $C_{\mathrm{Sf}}(\chi)$ is given by Eq. (83), $\chi_{0}=\left(\frac{3 f_{0} \lambda_{0}}{2}\right)^{\frac{1}{3}}$ is the initial void spacing ratio, and $\varepsilon_{\mathrm{d} s}=\varepsilon_{\mathrm{d} c}$ at $(\eta=0$, and $L=0)$ denotes the deviatoric equivalent plastic deformation at the onset of coalescence under pure shear loading. Combining Eqs. (108, 110) results into

$$
\xi=\frac{1-q_{1} f_{0} \exp \left(k_{\omega} \varepsilon_{\mathrm{d} s}\right)}{1-\chi_{0}^{2} \exp \left[\frac{2}{3}\left(\kappa+k_{\omega}\right) \varepsilon_{\mathrm{d} s}\right]} .
$$

The parameter $\varepsilon_{\mathrm{d} s}$ has a physical meaning, i.e. the onset of failure under pure shear, which could be identified from an experimental test or from unit cell computations, e.g the value of $\varepsilon_{\mathrm{d} s}$ was identified in the range of [0.8 1.2] in experiments of medium and high-strength steels performed by Barsoum and Faleskog (2007). Once $\varepsilon_{\mathrm{d} s}$ is known, Eq. (111) plays as a constraint on the constitutive parameters and allows estimating $\xi$ from $\varepsilon_{\mathrm{d} s}$ and other parameters.

\subsection{Ductility under proportional loading conditions}

In the absence of void nucleation, the evaluation of the void growth solution until the onset of coalescence requires the following parameters: $q_{1}, q_{2}, \kappa, f_{0}, \lambda_{0}, k_{\omega}, \eta_{s}$ and $\xi$. Initial spherical voids are considered with $\lambda_{0}=1$. In the following, without any other indications, $q_{1}=1.5, q_{2}=1, \kappa=1, f_{0}=0.005, k_{\omega}=0$, and $\eta_{s}=0.2$ are used and the value of $\xi=1.032$ is chosen to obtain $\varepsilon_{\mathrm{d} s}=0$ as a result of Eq. (111). At each given values of stress triaxiality $(\eta)$ and the Lode parameter $(L)$, the ductility function $\varepsilon_{\mathrm{d} c}=\varepsilon_{\mathrm{d} c}(\eta, L)$ is 


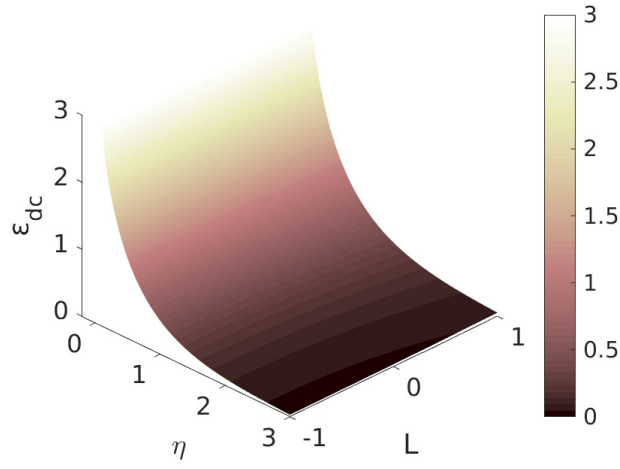

(a)

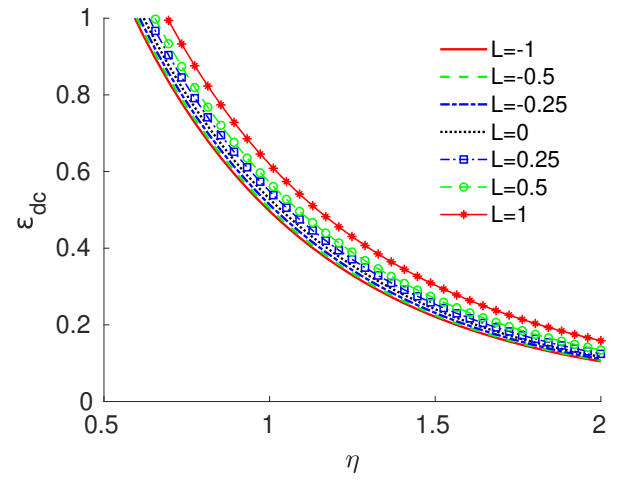

(b)

Figure 3: Internal necking coalescence: (a) failure surface and (b) extracted function of ductility depending on the stress triaxiality for various constant values of the Lode parameter.

easily deduced using the procedure described in Section 5.1. This function allows defining the failure surface in the $(\eta, L)$-space.

The failure surface predicted by the internal necking coalescence model alone is shown in Fig. 3(a), from which the triaxiality dependence of the ductility for various constant values of the Lode parameter is extracted and depicted in Fig. 3(b). The results not only evidence the $\eta$-dependence, i.e. the ductility decreases with increasing stress triaxiality, but also demonstrate the $L$-dependence: the ductility increases with the increase of the Lode parameter from $L=-1$, corresponding to the triaxial extension state, to $L=1$, corresponding to the triaxial compression state, although the $L$-dependence is not as strong as the $\eta$-dependence. As a result, the ductility is lower-bounded by the one predicted with $L=-1$ and upper-bounded by the one predicted with $L=1$. However, the internal necking model alone cannot correctly predict the ductility under shear-dominated conditions, in which the decrease of the ductility was experimentally observed ${ }^{6}$ (Bao and Wierzbicki, 2004; Barsoum and Faleskog, 2007; Barsoum et al., 2012; Faleskog and Barsoum, 2013).

Figs. 4(a) and (b) show respectively the failure surface predicted by the shear driven coalescence model alone and the triaxiality dependence of the ductility for various constant values of the Lode parameter extracted from this failure surface. The insensitivity to the sign of the Lode parameter is found as a result of using the maximum shear stress as the driven mechanism. The ductility is lower-bounded by the one predicted with $L=0$ and upper-bounded by the one predicted with $L= \pm 1$. The ductility is equal to 0 at $\eta=0$ and $L=0$ because $\varepsilon_{\mathrm{d} s}=0$ was assumed. If $\xi=1$ is employed, the model predicts zero ductility

\footnotetext{
${ }^{6}$ In the literature, depending on the materials, specimen geometries, boundary conditions, and measurement techniques, different conclusions on the influence of the stress state on the failure strain can be obtained. Under low stress triaxialities, a nonmonotonic dependence to the stress triaxiality (with a dip when decreasing stress triaxiality) was found by Bao and Wierzbicki (2004); Barsoum and Faleskog (2007); Barsoum et al. (2012); Faleskog and Barsoum (2013), while a monotonic dependence was found by Haltom et al. (2013); Ghahremaninezhad and Ravi-Chandar (2013); Papasidero et al. (2014). The origin of this difference is still an open question in the literature and goes beyond the scope of the present work.
} 


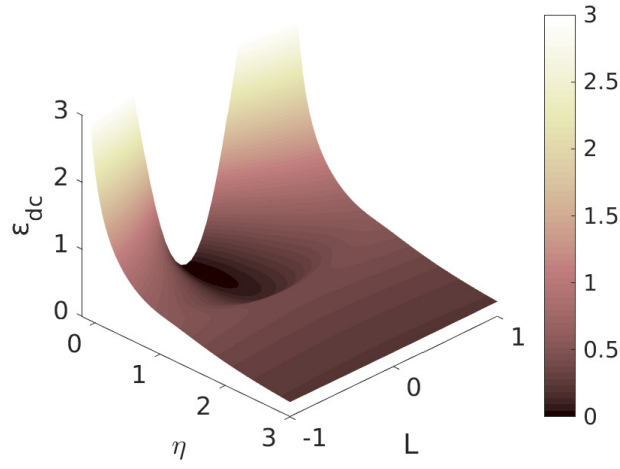

(a)

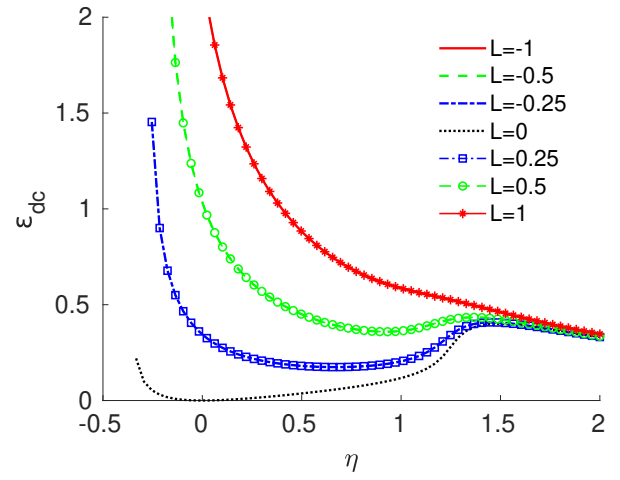

(b)

Figure 4: Shear dominated coalescence: (a) failure surface and (b) extracted function of ductility depending on the stress triaxiality for various constant values of the Lode parameter.

in a range of stress triaxiality, which is not physical and justifies the use of the parameter $\xi>1$. The obtained results in Figs. 4(a) and (b) show that the proposed shear driven coalescence model is able to reproduce the trend of the variation of ductility under low stress triaxialities with high $\eta$-dependence and high $L$-dependence as experimentally observed by Bao and Wierzbicki (2004); Barsoum and Faleskog (2007); Barsoum et al. (2012); Faleskog and Barsoum (2013). However, the shear driven coalescence model alone cannot correctly predict the $\eta$-dependence of the ductility at high stress triaxialities.

The failure surface predicted by the multi-surface model is shown in Fig. 5(a). The intersection between the failure surfaces predicted by the internal necking coalescence model and by the shear driven coalescence model is also reported. Although the internal necking coalescence model or the shear driven coalescence model alone cannot correctly predict the ductility in the entire range of the stress triaxiality and of the Lode parameter, in the multi-surface model, these two models compete, leading to the shear coalescence mode dominating at low stress triaxialities while the necking coalescence is the driving failure mode at high stress triaxialities. This trend agrees well with the experimental observations by Bao and Wierzbicki (2004); Barsoum and Faleskog (2007); Barsoum et al. (2012); Faleskog and Barsoum (2013). From the failure surface depicted in Fig. 5(a), one can extract the $\eta$ dependence of the ductility for various constant values of the Lode parameter as shown in Fig. 5 (b) and the $L$-dependence of the ductility for various constant values of the stress triaxiality as demonstrated in Figs. 5(c) and (d) under polar plots. At low stress triaxialities (e.g $\eta<0.5)$, the failure process is fully controlled by the shear coalescence model. At moderate stress triaxialities (e.g $1<\eta<1.2$ ), the competition is clearly observed, in which shear coalescence dominates at low absolute values of the Lode parameter and necking coalescence dominates at high absolute values of the Lode parameter. At high stress triaxialities (e.g $\eta>2$ ), only necking coalescence mode is activated.

The failure surface defines the admissible region, in which for each value of the stress triaxiality $\eta$, the ductility is upper-bounded and lower-bounded as $\varepsilon_{\mathrm{d} c \text {, min }}(\eta) \leq \varepsilon_{\mathrm{d} c}(\eta, L) \leq$ 


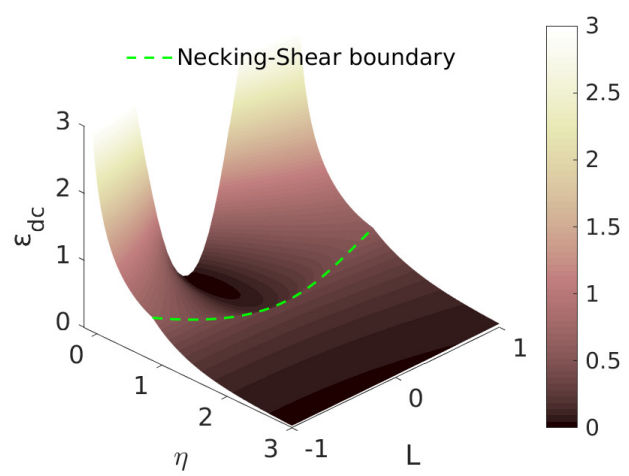

(a)

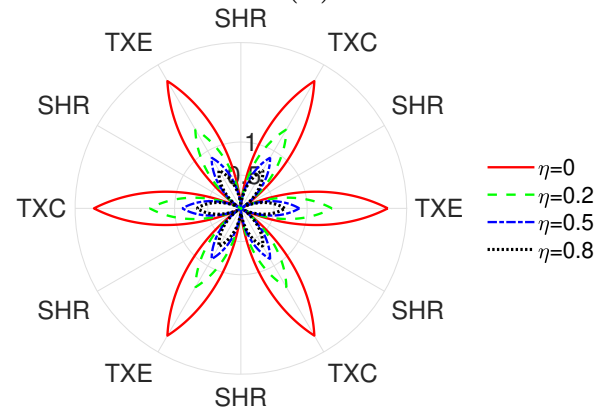

(c)

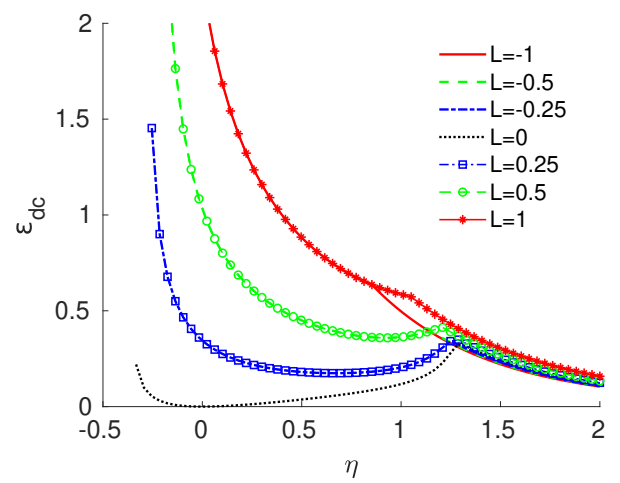

(b)

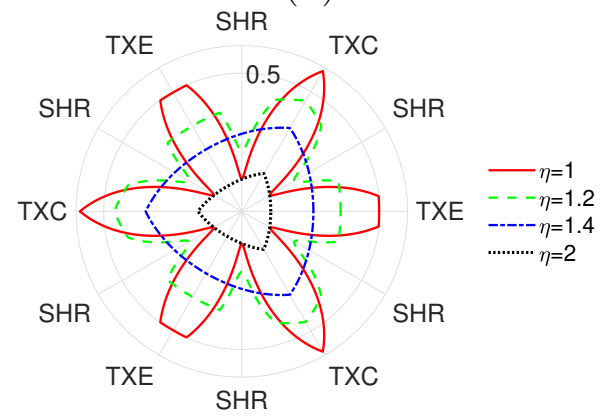

(d)

Figure 5: Multi-surface model: (a) failure surface with the intersection between the one predicted by the internal necking coalescence model alone and the one predicted by the shear driven coalescence model alone, (b) extracted function of ductility depending on the stress triaxiality for various constant values of the Lode parameter, (c) polar plot of the ductility predicted by the multi-surface model as a function of the Lode angle under various constant low values of the stress triaxiality, and (d) polar plot at various constant high values of the stress triaxiality. "TXE", "TXC", and "SHR" refer to "TriaXial Extension" $(L=-1)$, "TriaXial Compression" $(L=1)$, and "SHeaR" $(L=0)$, respectively.

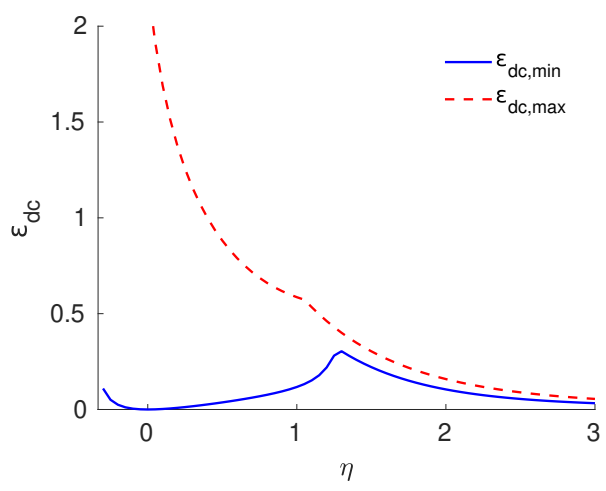

Figure 6: Admissible region predicted by the multi-surface model. 


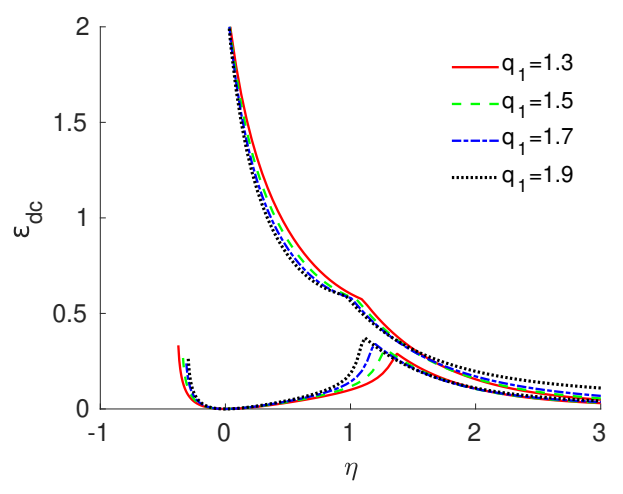

(a)

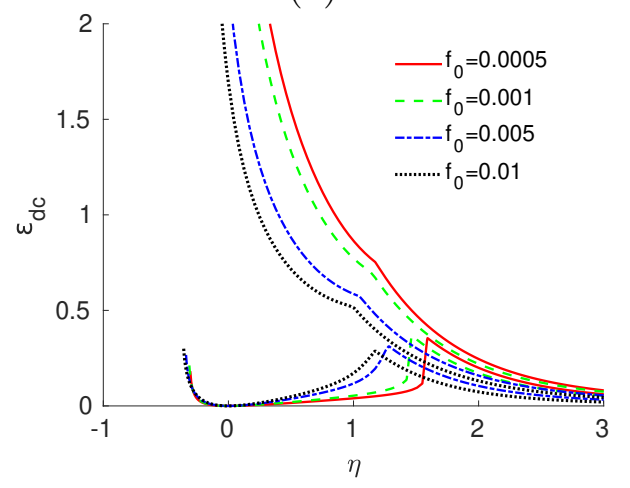

(c)

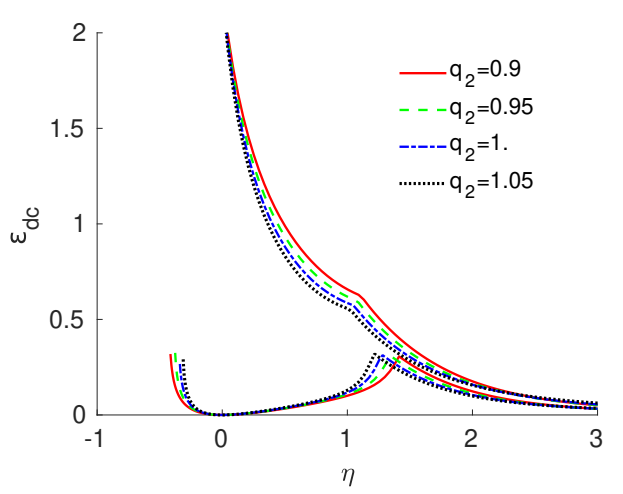

(b)

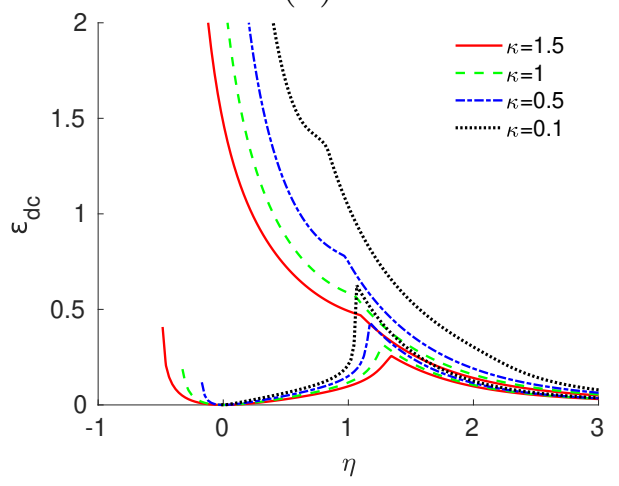

(d)

Figure 7: Dependence of the admissible region on: (a) $q_{1}$, (b) $q_{2}$, (c) $f_{0}$, and (d) $\kappa$.

$\varepsilon_{\mathrm{d} c, \max }(\eta)$ where

$$
\left\{\begin{array}{l}
\varepsilon_{\mathrm{d} c, \min }(\eta)=\min \left(\varepsilon_{\mathrm{d} c}(\eta, L), L \in[-11]\right), \text { and } \\
\varepsilon_{\mathrm{d} c, \max }(\eta)=\max \left(\varepsilon_{\mathrm{d} c}(\eta, L), L \in[-11]\right),
\end{array}\right.
$$

are respectively the upper-bound and lower-bound of the ductility. The admissible region is defined as the region delimited by these two bounds. In the case of internal necking coalescence, one has $\varepsilon_{\mathrm{d} c \text {, min }}(\eta)=\varepsilon_{\mathrm{d} c}(\eta,-1)$ and $\varepsilon_{\mathrm{d} c, \max }(\eta)=\varepsilon_{\mathrm{d} c}(\eta, 1)$. In case of the shear driven coalescence, one has $\varepsilon_{\mathrm{d} c, \min }(\eta)=\varepsilon_{\mathrm{d} c}(\eta, 0)$ and $\varepsilon_{\mathrm{d} c, \max }(\eta)=\varepsilon_{\mathrm{d} c}(\eta,-1)=\varepsilon_{\mathrm{d} c}(\eta, 1)$. The admissible region predicted by the multi-surface model is presented in Fig. (6), in which a more pronounced $L$-dependence of the ductility is observed at low stress triaxialities in comparison with the one at high stress triaxialities.

The influence of the constitutive parameters on the ductility is investigated in Fig. 7 and Fig. 8 in terms of the admissible region. The influence of the GTN constants $q_{1}$, and $q_{2}$ is noticeable mainly at moderate and high stress triaxialities and at high $|L|$ in the region with low stress triaxiality, see Figs. $7(\mathrm{a})$ and (b). Since $\kappa$ and $f_{0}$ are the main parameters governing the evolution of the void characteristics, they have a large effect on the ductility, especially, at moderate and high stress triaxialities and at high $|L|$, see Figs. 


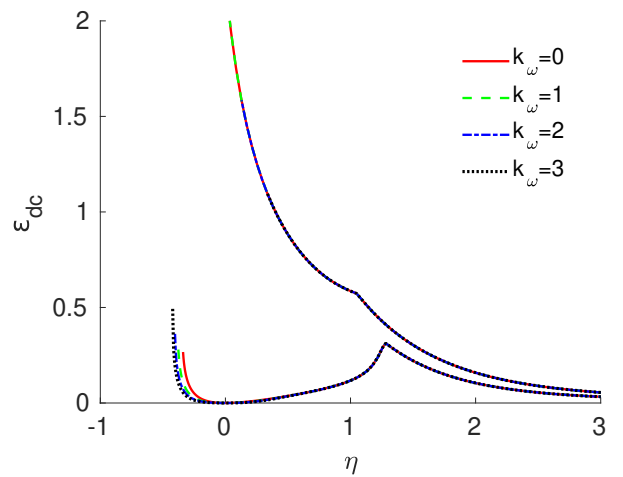

(a)

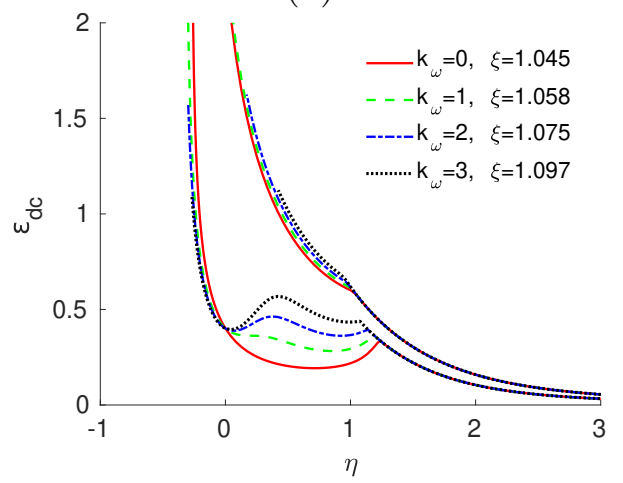

(c)

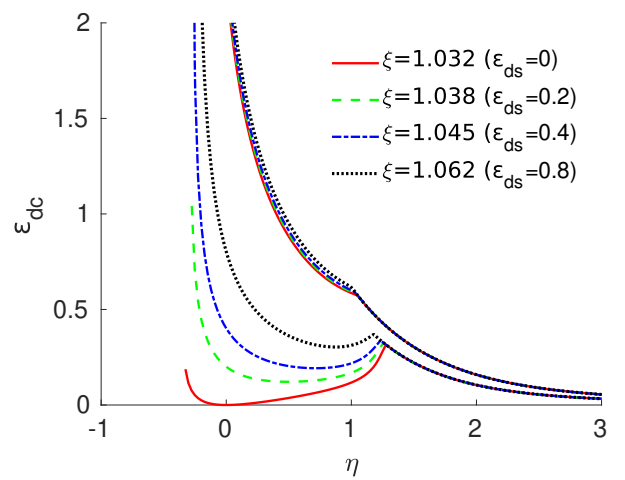

(b)

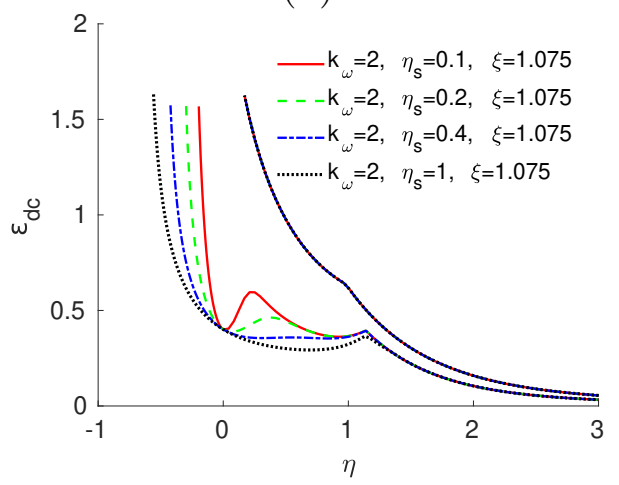

(d)

Figure 8: Dependence of the admissible region on: (a) $k_{\omega}$ with $\varepsilon_{\mathrm{d} s}=0$, (b) $\varepsilon_{\mathrm{d} s}$, (c) $k_{\omega}$ with $\varepsilon_{\mathrm{d} s}=0.4$, and (d) $\eta_{s}$ with $k_{\omega}=2$ and $\varepsilon_{\mathrm{d} s}=0.4$. 
7(c) and (d). In the case of low stress triaxiality and low $|L|$ (shear-dominated loading), the dominant parameters are $\xi$ (or $\varepsilon_{\mathrm{d} s}$ ), and $k_{\omega}$ together with $\eta_{s}$ as demonstrated in Fig. 8. When considering $\varepsilon_{\mathrm{d} s}=0, \xi$ is equal to 1.032 and independent to the value of $k_{\omega}$ as a result of Eq. (111). In this case, the admissible region is almost insensitive to the value of $k_{\omega}$, see Fig. 8(a). However, the admissible region is very sensitive to the value of $\varepsilon_{\mathrm{d} s}$, from which $\xi$ can be estimated using Eq. (111), as demonstrated in Fig. 8(b). When considering $\varepsilon_{\mathrm{d} s}>0$, the shape of the lower-bound of the admissible region is governed by $k_{\omega}$ and $\eta_{s}$, see Figs. $8(\mathrm{c})$ and $(\mathrm{d})$ with $\varepsilon_{\mathrm{d} s}=0.4$, and with $\xi$ estimated from $k_{\omega}, \varepsilon_{\mathrm{d} s}$, and other parameters using Eq. (111). From this sensitivity analysis, a suitable calibration plan can be developed to identify the constitutive parameters in an efficient way.

\subsection{Comparison with unit cell simulations}

The ductility values predicted by the present model are compared to the finite element unit cell simulations provided by Dunand and Mohr (2014). The unit cell contains an initial spherical void at the center with an initial porosity $f_{0}=7 \times 10^{-3}$ and an initial void spacing $\lambda_{0}=2$. The unit cell simulations performed under stress triaxiality values $\eta=0.3$ and $\eta=0.5$ are considered with different values of the Lode variable.

To ascertain the validity of the equivalence (64) used in the present model, we consider $q_{1}=1.5, q_{2}=1$, and $\eta_{s}=1$ while varying $k_{\omega}$ and $\kappa$ in order to recover the ductility observed by the unit cell simulations. Since the parameter $\varepsilon_{\mathrm{d} s}$ has as physical meaning, which is the strain at the onset of failure under pure shear, its value is fixed in this study: two values, 0.5 and 0.8 , of $\varepsilon_{\mathrm{d} s}$ are successively considered.

The failure strain obtained with the unit cell simulations given by Dunand and Mohr (2014) is defined based on the integral of the plastic work over the whole unit cell. Therefore the mean equivalent plastic strain of the matrix at the onset of coalescence (denoted by $\varepsilon_{\mathrm{m} c}$ ) predicted by the present model is considered as an equivalent quantity. The results in Figs. $9(\mathrm{a}),(\mathrm{b}),(\mathrm{c})$ and (d) show that the present model, although considering a simplified void growth assumption, is able to capture the strain at the onset of failure predicted by the unit cell simulations, except for states close to the triaxial compression (TXC).

However, under low stress triaxialities, the voids could become so prolate (Pardoen and Hutchinson, 2000) that the void growth cannot be correctly captured by an "equivalent" spherical voids as assumed in Eq. (64). An obvious but complex solution would be to consider the extended void growth version proposed by Gologanu et al. (1994, 1993). Another solution is to consider the $q_{2}$ parameter in the context of the simplified void growth assumption allowing to artificially account for the effect of void shape.

\section{Numerical applications}

This section presents some representative numerical examples. Owing to the nonlocal formulation, the finite element resolution is mesh-independent. Furthermore, the proposed model has the capability to capture the slant failure mode in the plane strain specimens, shear failure in "V-notched" plates under shearing, and the cup-cone failure scenario in the axisymmetric smooth and notched bars. 

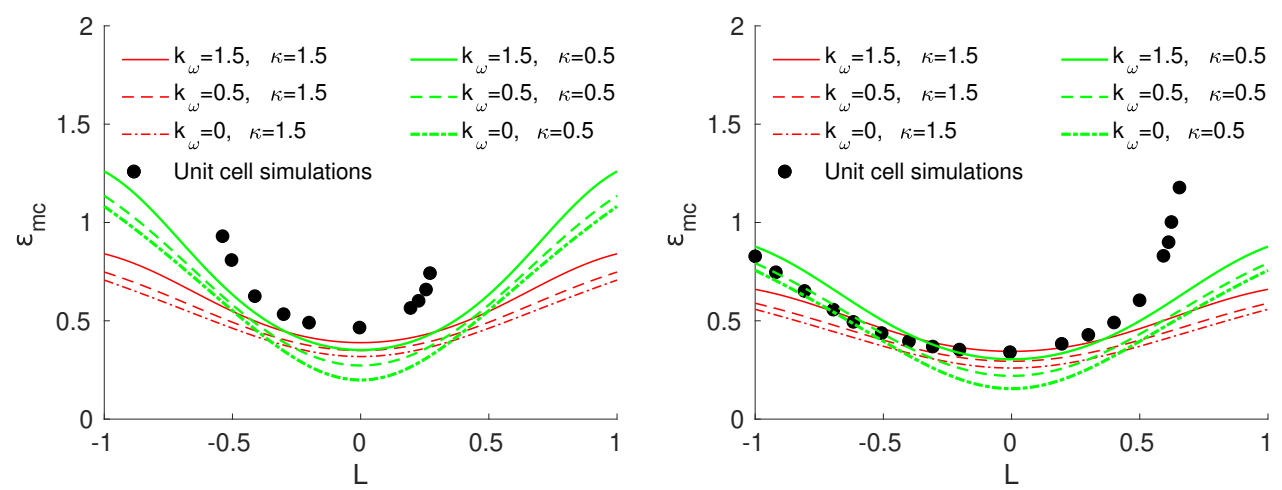

(a) $\varepsilon_{\mathrm{d} s}=0.5$ and $\eta=0.3$

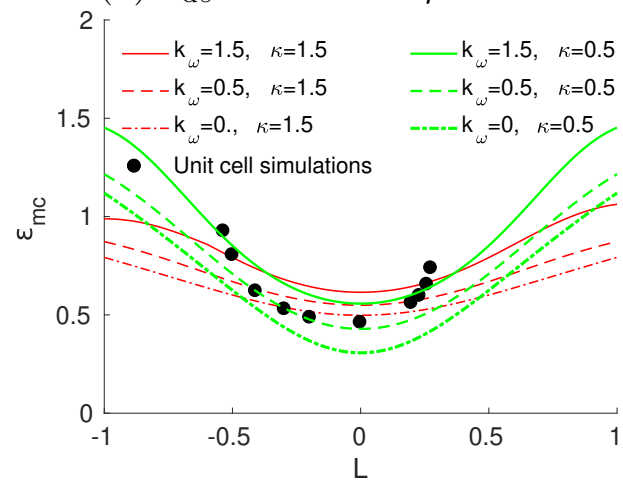

(b) $\varepsilon_{\mathrm{d} s}=0.5$ and $\eta=0.5$

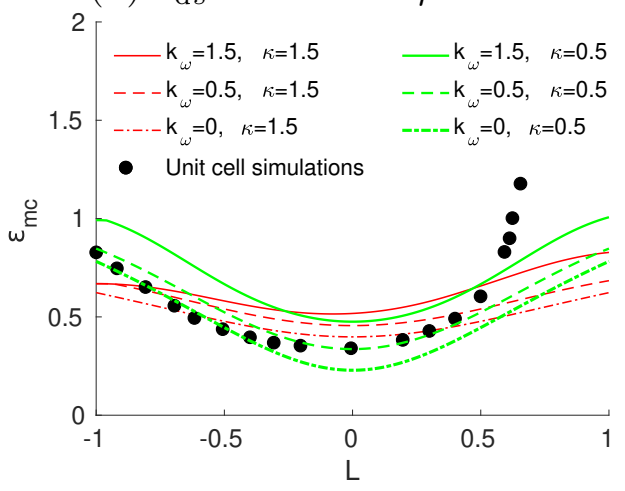

(c) $\varepsilon_{\mathrm{d} s}=0.8$ and $\eta=0.3$

(d) $\varepsilon_{\mathrm{d} s}=0.8$ and $\eta=0.5$

Figure 9: Mean equivalent plastic strain of the matrix at the onset of coalescence $\varepsilon_{\mathrm{m} c}$ predicted by the present model as function of the stress state and material parameters: (a) $\varepsilon_{\mathrm{d} s}=0.5$ and $\eta=0.3$, (b) $\varepsilon_{\mathrm{d} s}=0.5$ and $\eta=0.5$, (c) $\varepsilon_{\mathrm{d} s}=0.8$ and $\eta=0.3$, and (d) $\varepsilon_{\mathrm{d} s}=0.8$ and $\eta=0.5$. The results of the unit cell simulations provided by Dunand and Mohr (2014) at $\eta=0.3$ and $\eta=0.5$ are also reported. 
Table 2: Material parameters used for numerical analyses

\begin{tabular}{l|l}
\hline Elastoplastic parameters & $E=210 \mathrm{GPa}, \nu=0.3, \sigma_{\mathrm{Y}}^{0}=420 \mathrm{MPa}$, \\
& $\varepsilon_{0}=0.002, \alpha=0.15$ \\
\hline Initial void characteristics & $f_{0}=5 \times 10^{-4}, \lambda_{0}=1, \chi_{0}=0.0909$ \\
\hline Porosity related parameters & $q_{1}=1.5, q_{2}=1, A_{n}=0, k_{\omega}=0, \kappa=1$, \\
& $h=0.1, g=1.24, \xi=1.015, K_{\chi}=5$ \\
\hline Nonlocal parameters & $l_{\varepsilon_{\mathrm{v}}}=l_{\varepsilon_{\mathrm{d}}}=l_{\varepsilon_{\mathrm{m}}}=l=100 \mu \mathrm{m}$ \\
\hline
\end{tabular}

The strain hardening of the matrix material obeys a power law in terms of the equivalent plastic strain of the matrix

$$
\sigma_{\mathrm{Y}}=\sigma_{\mathrm{Y}}^{0}\left(1+\frac{\varepsilon_{\mathrm{m}}}{\varepsilon_{0}}\right)^{\alpha},
$$

where $\sigma_{\mathrm{Y}}^{0}$ is the initial yield stress, $\varepsilon_{0}$ is a reference strain, and $\alpha$ is the strain hardening exponent. Unless otherwise stated, void growth by nucleation and by shear are deactivated by setting $A_{n}=0$ and $k_{\omega}=0$. The initial void spacing parameter $\lambda_{0}=1$ is chosen indicating an isotropic material. Eq. (62) allows estimating $\chi_{0}=\left(1.5 f_{0} \lambda_{0}\right)^{1 / 3}=0.0909$. The value $\xi=1.015$ is used, leading to $\varepsilon_{\mathrm{d} s}=0.95$ as a result of Eq. (111). The assumption $l_{\varepsilon_{\mathrm{v}}}=l_{\varepsilon_{\mathrm{d}}}=l_{\varepsilon_{\mathrm{m}}}=l$ is adopted for simplicity. Unless otherwise stated, the material parameters detailed in Tab. 2 are used.

When dealing with problems involving material softening, the load drops fast under strain localization and the convergence of a resolution method based on a prescribed force or displacement cannot be guaranteed, and totally fails if snap-back occurs. In order to improve the convergence and to handle the snap-back loading path which possibly occurs, the arc-length path following technique (Riks, 1979, 1992; Geers, 1999) is considered. The numerical implementation of the monolithic nonlocal finite element resolution in the context of an arc-length path following technique is briefly recalled in Appendix J.

\subsection{Plane strain tension specimen}

This section investigates the failure of a plane strain tension specimen. The geometry and loading conditions are sketched in Fig. 10. The specimen dimensions are chosen as $L=12.5 \mathrm{~mm}$ and $e_{0}=3 \mathrm{~mm}$. A very small thickness reduction $(0.5 \%)$ is introduced in the central section to trigger the localization process. The mesh sensitivity is investigated with four different meshes: (a) very coarse mesh consisting of 2040 elements, (b) coarse mesh consisting of 3250 elements, (c) medium mesh consisting of 9120 elements, and (d) fine mesh consisting of 17800 elements, see Figs. 11(a), (b), (c), and (d) respectively. In all meshes, six-node triangular elements under-integrated by three Gauss points are used. The entire specimen is modeled in order to capture the slant failure mode that destroys the symmetry of the problem.

Fig. 12(a) plots the force-thickness reduction curves in terms of the nominal axial stress $\left(F / e_{0}\right)$ versus the relative thickness reduction $\left(-\Delta e / e_{0}\right)$. The results are mesh insensitive. In 


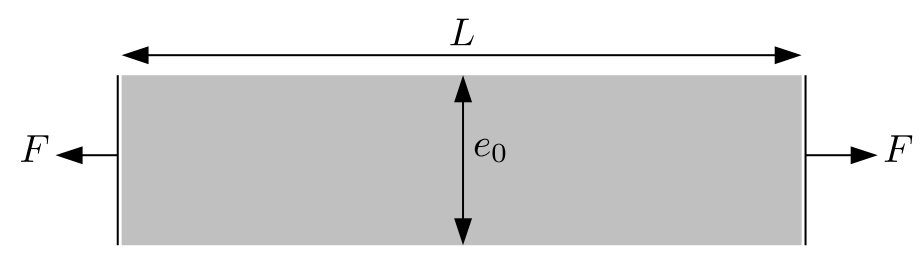

Figure 10: Plane strain tension specimen - geometry and boundary conditions.

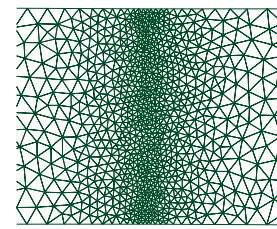

(a)

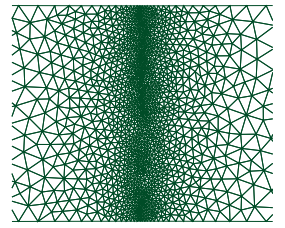

(b)

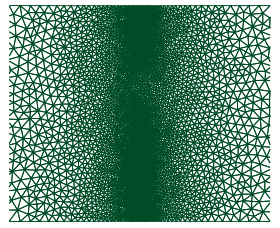

(c)

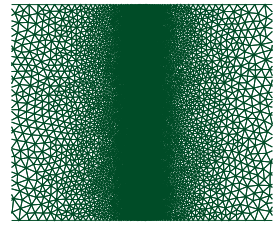

(d)

Figure 11: Plane strain tension specimen - different finite element meshes in the central region of the specimen for the mesh sensitivity analysis: (a) very coarse mesh, (b) coarse mesh, (c) medium mesh, and (d) fine mesh.

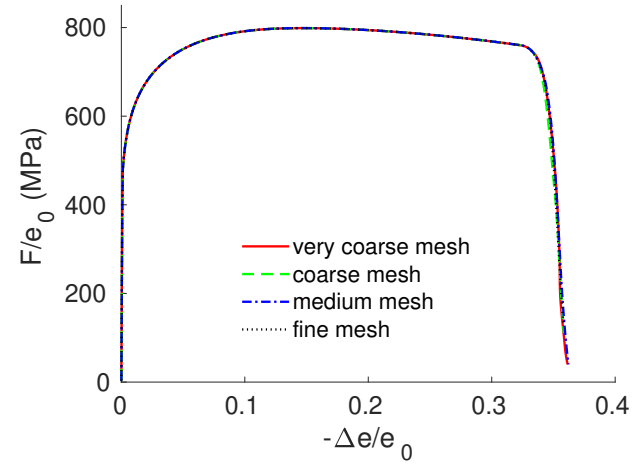

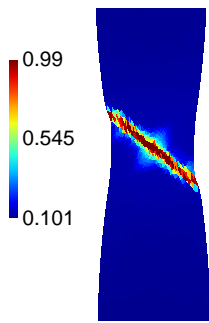

(b)

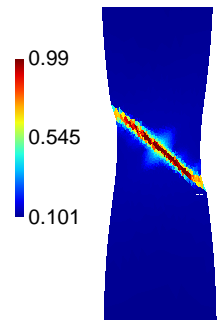

(c)

(a)

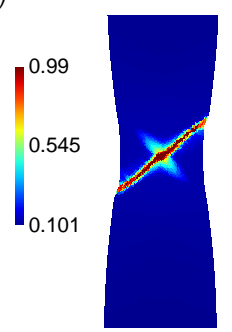

(d)

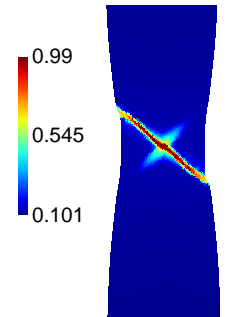

(e)

Figure 12: Plane strain tension specimen - mesh sensitivity: (a) force-thickness reduction curves and distribution of the void ligament ratio $\chi$ at the last converged step with (b) the very coarse mesh, (c) the coarse mesh, (d) the medium mesh, and (e) the fine mesh. 


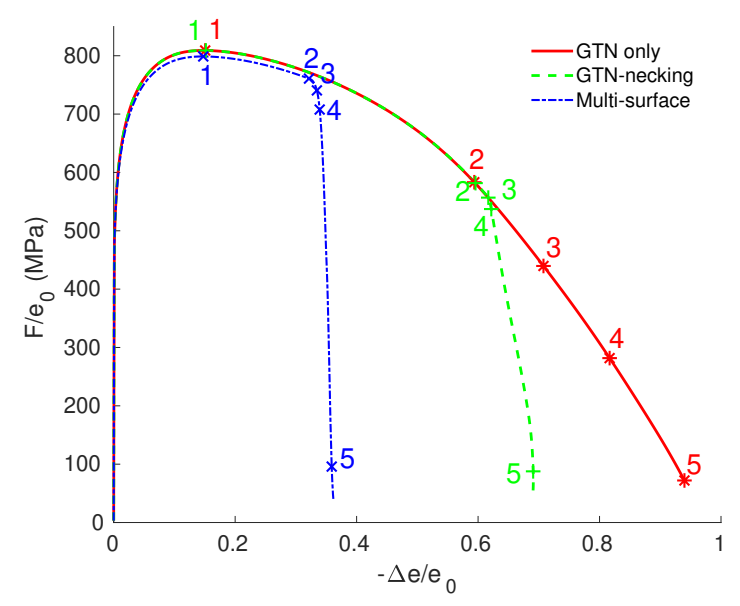

Figure 13: Plane strain tension specimen - effect of the constitutive model. Several points are marked for which the distributions of the active plasticity and of the active coalescence states are extracted.

this simulation, the Lode parameter is close to 0 and the stress triaxiality remains moderate. As a result, the failure is driven by shear coalescence. Prior to the shear band formation, the plastic flow first develops homogeneously, and then localizes inside a diffuse necking region. As the void ligament ratio (denoted by $\chi$ ) is the damage indicator in the coalescence-driven failure mechanism, Figs. 12(b)-(e) show its distribution at the last converged step, which corresponds to the last point in the force-thickness reduction curves depicted in Fig. 12(a). Since material discontinuity is not considered, a critical value $\chi=0.99$ is chosen to represent the ultimate failure state. The localization band thickness is insensitive to the mesh size and follows a slanted path, which is consistent with the experimental observations (Besson et al., 2003). The coarse mesh is used in the subsequent simulations.

\subsubsection{Cooperation between localization and coalescence}

As observed by Besson et al. (2003), the GTN model is able to capture the slant failure mode when triggering a sufficiently high void growth rate, which can be generated either by introducing a second population of voids through a large enough void nucleation contribution or by using a large value of the $q_{2}$ parameter of the GTN yield surface. In the present model, the slant failure path emerges without the need of any artificial acceleration of the void growth process. To demonstrate this capability, the influence of the constitutive model is investigated by considering the GTN model only, the GTN model coupled with internal necking coalescence, and the full model, in which the GTN model is coupled with both internal necking and shearing coalescence mechanisms. The force-thickness reduction curves are shown in Fig. 13. In the absence of void coalescence, the GTN model predicts a very high ductility. When both void coalescence modes are considered, the shear driven coalescence mode is preferred since it leads to a lower ductility than the one obtained when only the internal necking coalescence mode is allowed. The interesting question addressed is whether the shear banding process precedes or follows the onset of coalescence and how they influence one another. The dominating failure mechanism is investigated by analyzing the two following sets of material points: 


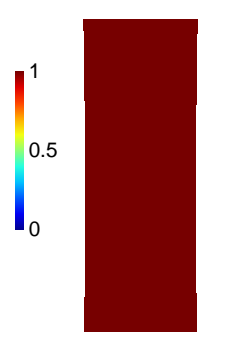

(a) $1-\mathrm{APZ}$

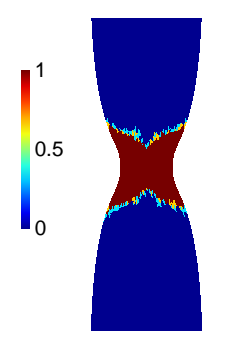

(b) 2 -APZ

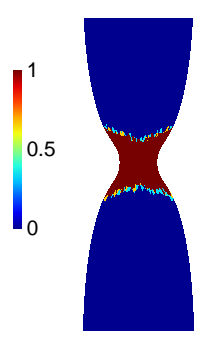

(c) $3-\mathrm{APZ}$

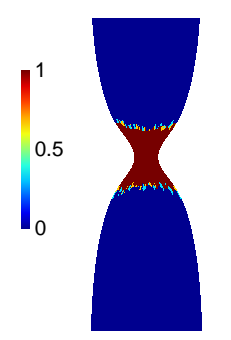

(d) $4-\mathrm{APZ}$

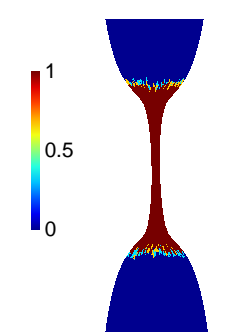

(e) 5 -APZ

Figure 14: Plane strain tension specimen - GTN model only: the active plastic zone (APZ) at the different loading stages 1-5 reported in Fig. 13.

- The active plastic zone (APZ) consisting of all material points in which plasticity is active, i.e. $\dot{\varepsilon}_{\mathrm{m}}(\mathbf{x})>0$; and

- The active coalescence zone (ACZ) consisting of all material points in which one of the coalescence yield surfaces is active, i.e. $\Phi_{\mathrm{S}}(\mathbf{x})=0$ or $\Phi_{\mathrm{T}}(\mathbf{x})=0$. Note that one always has $\Phi_{\mathrm{S}}(\mathbf{x})<0$ if the shear driven coalescence mode is prohibited.

Figure 14 shows the active plastic zone in the case when only the GTN model is considered. The shear banding process does not occur and the necking process proceeds down to a point, see Fig. 14(e). The results with the GTN model combined with the internal necking coalescence mode are presented in Fig. 15. A crack develops inside the necking region and the failure process continues with a relatively smooth evolution of the necking. With the full multi-surface model, a shear band is formed and the slant failure path is observed, see Fig. 16. In this case, the first coalescence event starts before the development of the shear band, see Fig. 16(g). The shear band is thus triggered by the occurrence of a shear coalescence event. The shear band develops and the failure process continues inside by propagation of a slant crack through a repetition of shear coalescence events near the crack tip region. More detailed analysis of these aspects of competition versus cooperation between localization and coalescence dominated failure, see also the work by Tekoğlu et al. (2015), will be addressed in a future investigation.

\subsubsection{Sensitivity analysis}

As demonstrated in Fig. 8(b), the parameter $\varepsilon_{\mathrm{d} s}$ plays an important role in controlling the onset of the shear driven coalescence under shear-dominated loading conditions. With the known value of $\varepsilon_{\mathrm{d} s}$ and other constitutive parameters, the value of $\xi$ can be estimated using Eq. (111). The case without considering the shear driven coalescence mode can be achieved using a very large value of $\xi$. The effect of this parameter on the numerical results is investigated in Fig. 17. The force-thickness reduction curves are plotted in Fig. 17 (a) for different values of $\varepsilon_{\mathrm{d} s}$ showing a negligible effect up to the onset of coalescence at the center of the specimen since the pre-coalescence stage is only governed the GTN yield surface. A larger value of $\xi$ (same role as $\varepsilon_{\mathrm{d} s}$ ) leads to an earlier localization initialization and alleviates the slant fracture mode, see Figs. 17(b)-(g). Once the localization process has started, multiple localization bands nucleate at the center of the specimen at approximately 

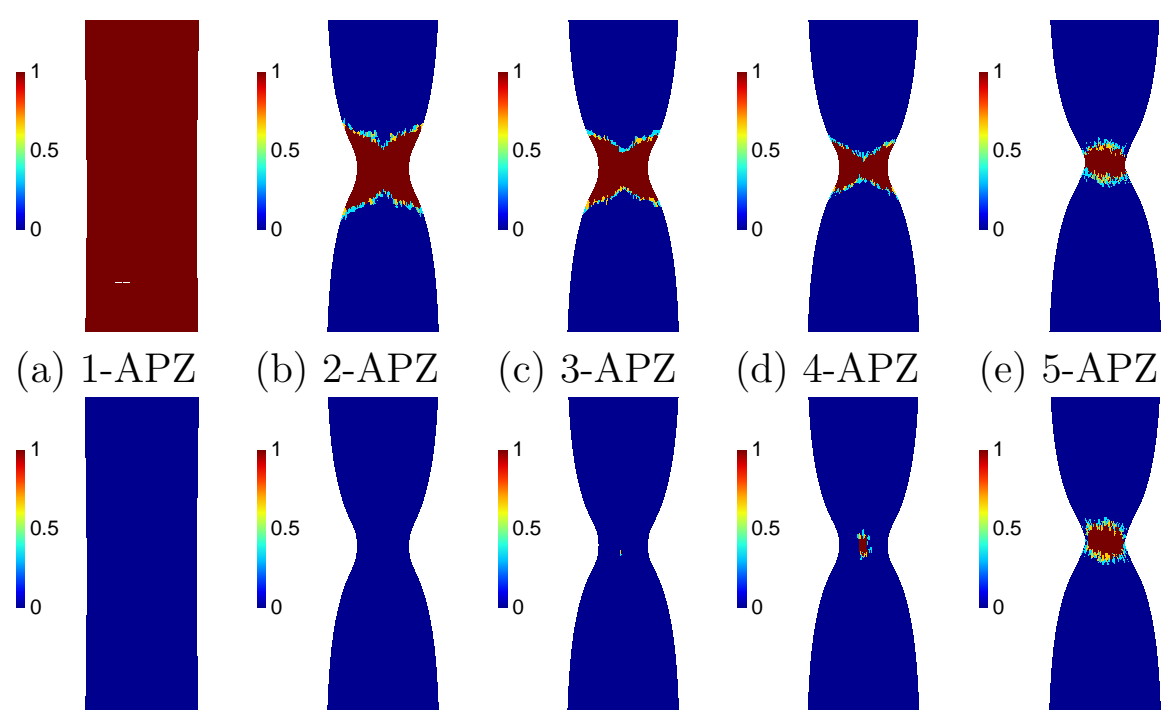

(b) $2-\mathrm{APZ}$

(c) $3-\mathrm{APZ}$

(d) 4 -APZ

(e) 5 -APZ

(f) $1-\mathrm{ACZ}$
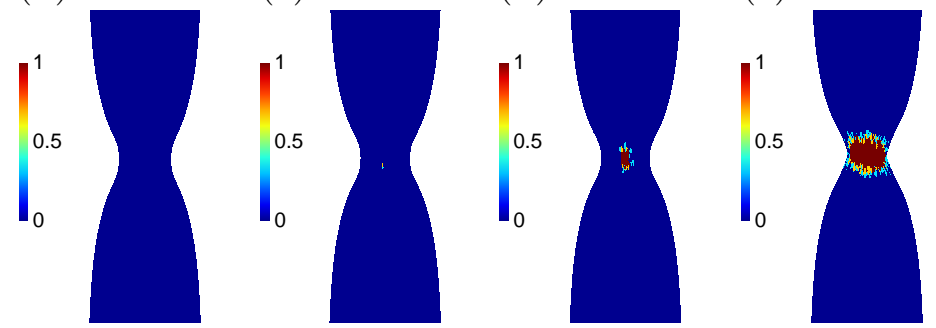

(g) $2-\mathrm{ACZ}$

(h) 3 -ACZ

(i) 4 -ACZ

(j) $5-\mathrm{ACZ}$

Figure 15: Plane strain tension specimen - GTN with internal necking: the active plastic zone (APZ) and active coalescence zone (ACZ) at the different loading stages 1-5 reported in Fig. 13.
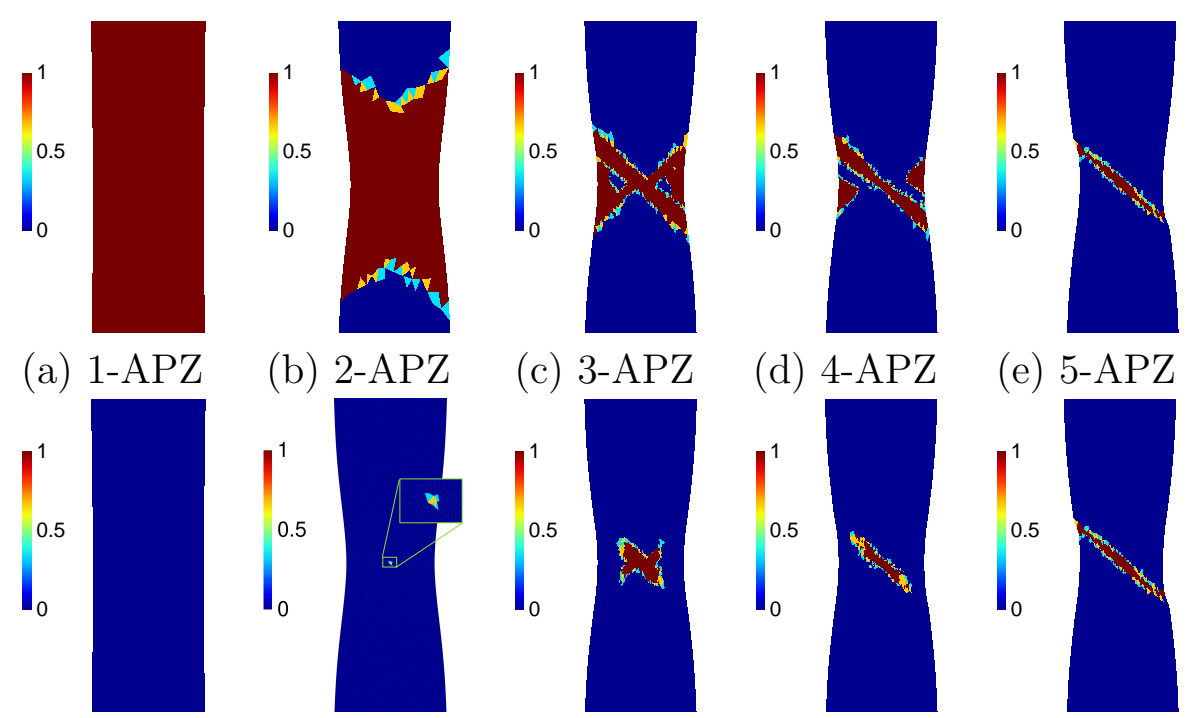

(b) 2-APZ

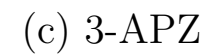

(d) 4-APZ

(e) 5-APZ

(f) $1-\mathrm{ACZ}$
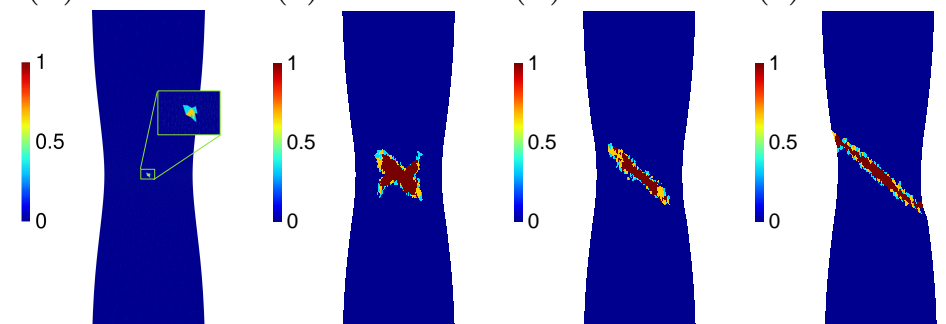

(g) 2-ACZ

(h) 3 -ACZ

(i) $4-\mathrm{ACZ}$

(j) 5 -ACZ

Figure 16: Plane strain tension specimen - multi-surface model: the active plastic zone (APZ) and active coalescence zone (ACZ) at the different loading stages 1-5 reported in Fig. 13. 


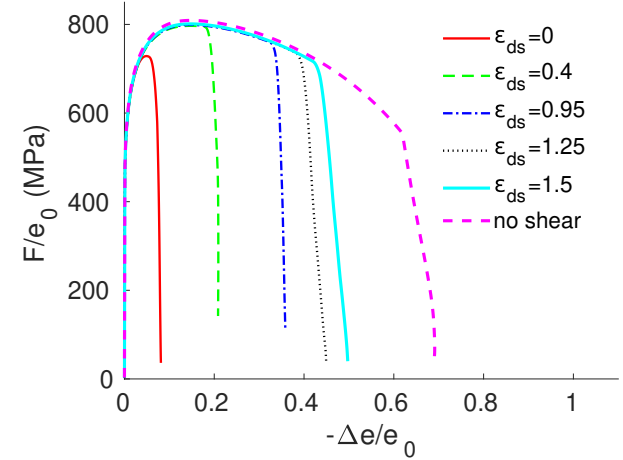

(a)

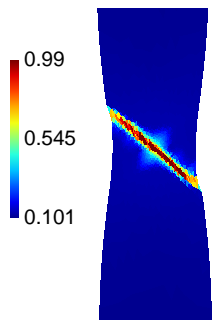

(d) (e)

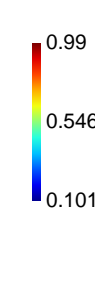

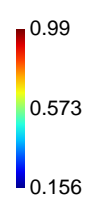

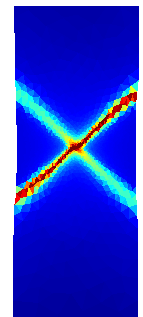

(b)

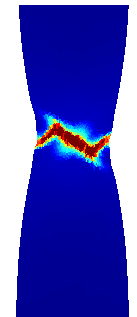

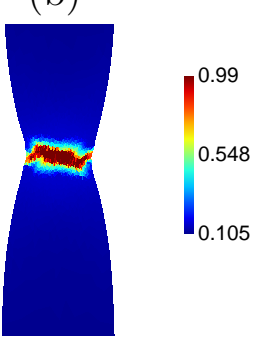

(f)

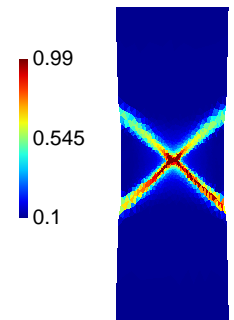

(c)

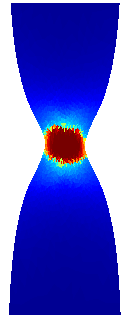

(g)

Figure 17: Plane strain tension specimen - sensitivity to $\varepsilon_{\mathrm{d} s}$ : (a) force-thickness reduction curves and distribution of the void ligament ratio $\chi$ at the last converged step with (b) $\varepsilon_{\mathrm{d} s}=0$ (corresponding to $\xi=1.0076$ ), (c) $\varepsilon_{\mathrm{d} s}=0.4$ (corresponding to $\xi=1.0101$ ), (d) $\varepsilon_{\mathrm{d} s}=0.95$ (corresponding to $\xi=1.015$ ), (e) $\varepsilon_{\mathrm{d} s}=1.25$ (corresponding to $\xi=1.0186$ ), (f) $\varepsilon_{\mathrm{d} s}=1.5$ (corresponding to $\xi=1.0222$ ), and (g) without possible shear coalescence (corresponding to $\xi=+\infty$ ). 


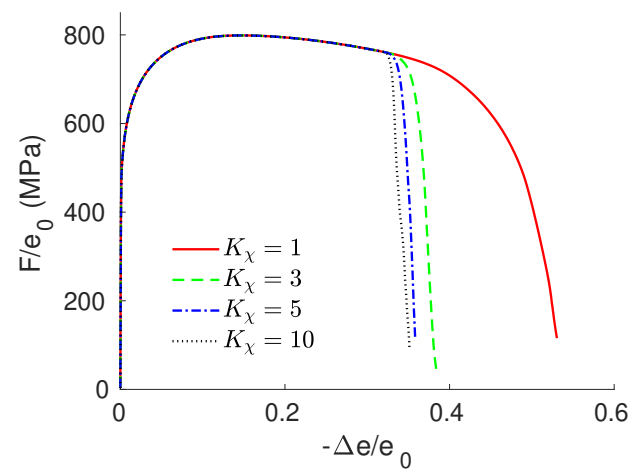

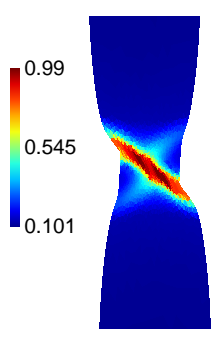

(b)

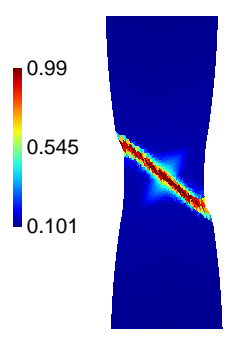

(c)

(a)

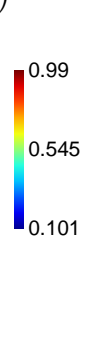

(d)

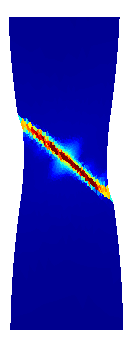

d)

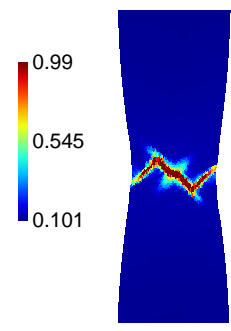

(e)

Figure 18: Plane strain tension specimen - sensitivity to $K_{\chi}$ : (a) force-thickness reduction curves and distribution of the void ligament ratio $\chi$ at the last converged step with (b) $K_{\chi}=1$, (c) $K_{\chi}=3$, (d) $K_{\chi}=5$, and (e) $K_{\chi}=10$.

$45^{\circ}$ to the loading direction. The localization band can propagate following non-symmetric or symmetric configurations. If the value of $\xi$ is large enough, the localization band deviates at the region close to the free surface, see Figs. 17(e) and (f). When $\xi$ becomes very large, the shear-driven coalescence mechanism cannot occur and the slant fracture mode cannot be reproduced as shown in Fig. 17(g).

The effect of $K_{\chi}$ is also investigated in Figs. 18(a)-(e). Larger values of $K_{\chi}$ mean a stronger void ligament growth rate, leading to a thinner localization band and a faster drop in the softening parts of the force-thickness reduction curves. Moreover, a deviation of the localization band appears when $K_{\chi}$ is sufficiently large, see Fig. 18(e).

The present multi-surface model can model size effect through the nonlocal length scale $l$ as a characteristic of a nonlocal framework. The force-thickness reduction curves are reported in Fig. 19(a) for different values of the nonlocal length. The effect of the nonlocal length on the behavior prior to the initiation of the localization band remains negligible but is considerable during the localization stage. Figs. 19(b)-(d) show the distribution of the void ligament ratio $\chi$ at the last converged step corresponding to the last point of the curves in Fig. 19(a). When decreasing the nonlocal length, the load drops faster and the width of the localization band becomes thinner, evidencing a less ductile behavior.

\subsection{V-notched specimen under shear}

This section investigates the failure of a V-notched specimen under shear loading. The geometry and loading conditions are described in Fig. 20. The specimen dimensions are 


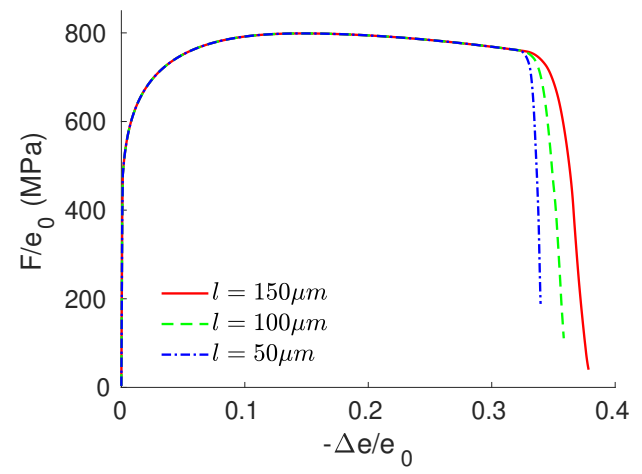

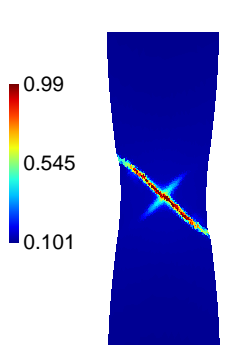

(b) (a)

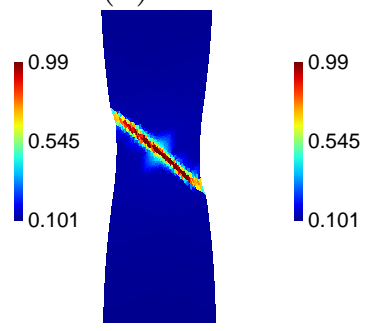

(c)

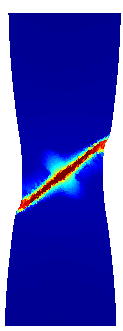

(d)

Figure 19: Plane strain specimen - size effect: (a) force-thickness reduction curves and distribution of the void ligament ratio $\chi$ at the last converged step with (b) $l=50 \mu \mathrm{m}$, (c) $l=100 \mu \mathrm{m}$, and (d) $l=150 \mu \mathrm{m}$.

chosen as $L=4.5 \mathrm{~mm}, H=1.50 \mathrm{~mm}, e_{0}=1 \mathrm{~mm}$, and the notch angle equal to $45^{\circ}$. In this example, the Lode parameter is close to 0 and the stress triaxiality remains low. Consequently, the failure is driven by shear coalescence.

\subsubsection{Mesh sensitivity and choice of nonlocal variables}

The mesh sensitivity is investigated by considering three different meshes: (a) a coarse mesh consisting of 674 elements, (b) a medium mesh consisting of 2094 elements, and (c) a fine mesh consisting of 6662 elements, see Figs. 21(a), (b), and (c) respectively. For all meshes, six-node triangular elements under-integrated by three Gauss points are used.

Since the current framework considers two distinct softening indicators: $f$ and $\chi$, their

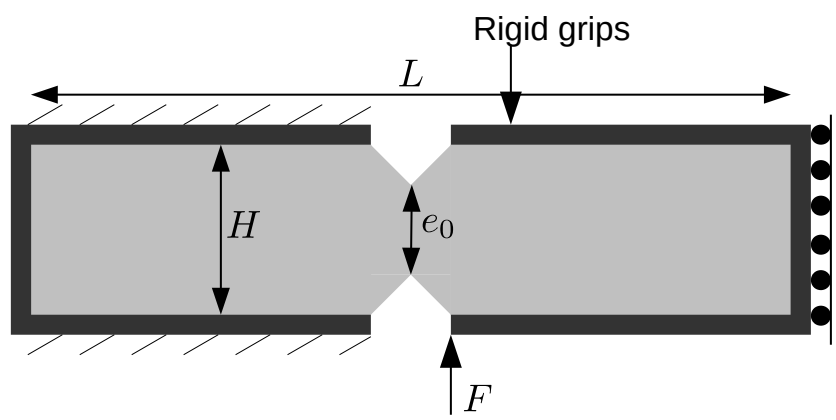

Figure 20: V-notched specimen - geometry and boundary conditions. 


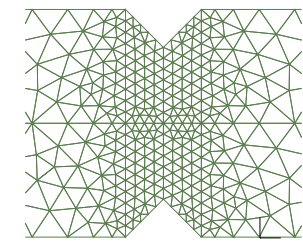

(a)

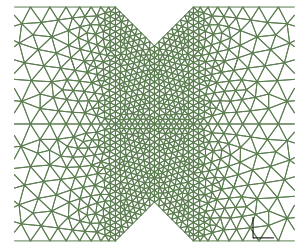

(b)

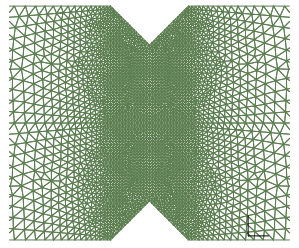

(c)

Figure 21: V-notched specimen - the different finite element meshes for the mesh sensitivity analysis: (a) coarse mesh, (b) medium mesh, and (c) fine mesh.

nonlocality needs to be considered to resolve the mesh-dependency issue. Whenever the void coalescence occurs, the nonlocality of $\chi$ is mandatory since it is the only parameter governing the failure process. In the present framework, the nonlocal plastic state, i.e. the nonlocality of $\varepsilon_{\mathrm{v}}$, of $\varepsilon_{\mathrm{d}}$, and of $\varepsilon_{\mathrm{m}}$, is considered in order to achieve full regularization. Fig. 22(a) illustrates the force-displacement curves in terms of the nominal shear stress $\left(F / e_{0}\right)$ versus the relative vertical displacement $\left(U_{y} / e_{0}\right)$. The results are mesh-insensitive owing to the non-locality in $\varepsilon_{\mathrm{d}}$. Figs. $22(\mathrm{~b})-(\mathrm{d})$ show the distribution of the void ligament ratio (denoted by $\chi$ ) at the last converged step, which corresponds to the last point in the force-displacement curves depicted in Fig. 22(a). The localization band follows the loading direction and its thickness converges with respect to the mesh size.

If only $\varepsilon_{\mathrm{v}}$ were chosen as the nonlocal variable, the mesh dependency would not be resolved in the case of shear coalescence-controlled failure as shown in Fig. 23.

Failure governed by void nucleation is now artificially produced by increasing the nucleation intensity to $A_{n}=0.2$ and considering $\xi$ as being sufficiently large in order not to trigger shear coalescence. When considering only $\varepsilon_{\mathrm{v}}$ as the sole nonlocal variable, mesh sensitivity is observed in Fig. 24(a), while mesh-independent results are obtained using the current framework with three nonlocal variables, see Fig. 24(b) because of the non-locality of $\varepsilon_{\mathrm{m}}$.

Finally, nonlocality of the volumetric plastic strain $\varepsilon_{\mathrm{v}}$ is necessary for cases involving high stress triaxiality, in which the void growth is mainly controlled by this mechanism and the localization could occur before void coalescence by internal necking as pointed out by Tekoğlu et al. (2015).

Another possibility to generate a nonlocal plastic formulation is to consider a strain gradient based plasticity approach (De Borst and Mühlhaus, 1992) by incorporating a Laplacian term of the hardening variable in the yield condition as considered by Zhang et al. (2018). However, this approach belongs to the family of explicit nonlocal methods, which are not truly nonlocal as pointed out by Peerlings et al. (2001). Indeed, in this family of models, the gradient terms of order higher than two have been neglected whereas these terms are indirectly accounted for in the implicit formulation considered in the present framework. Moreover, because for explicit nonlocal methods the nonlocal variable at a point depends only on the local value and their gradients at the same point, spatial interactions are therefore limited to an infinitesimal neighborhood (Peerlings et al., 2001). 


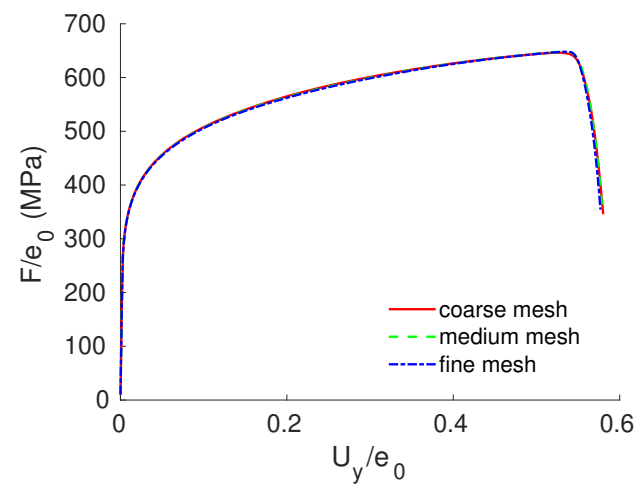

(a)

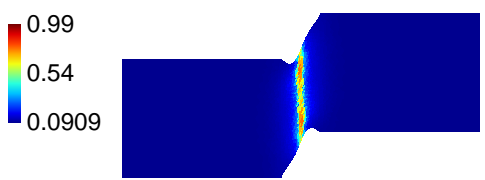

(c)

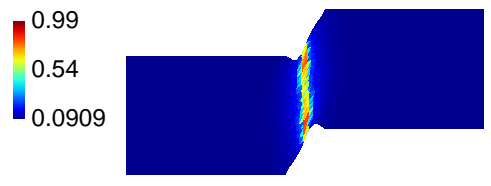

(b)

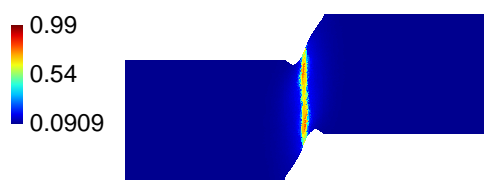

(d)

Figure 22: V-notched specimen - mesh sensitivity: (a) force-displacement curves, and distribution of the void ligament ratio $\chi$ at the last converged step for (b) the coarse mesh, (c) the medium mesh, and (d) the fine mesh.

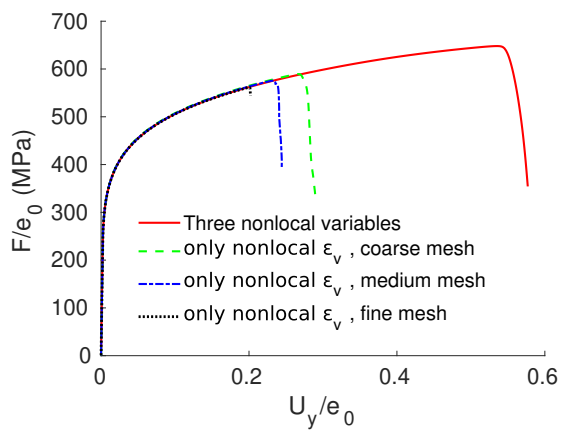

Figure 23: V-notched specimen - with as sole nonlocality the volumetric plastic strain $\varepsilon_{\mathrm{v}}$. 


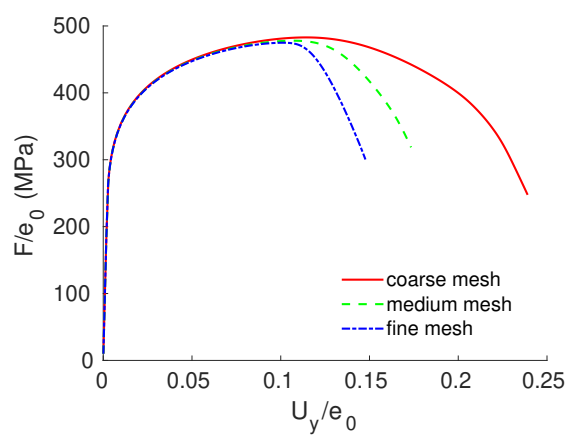

(a)

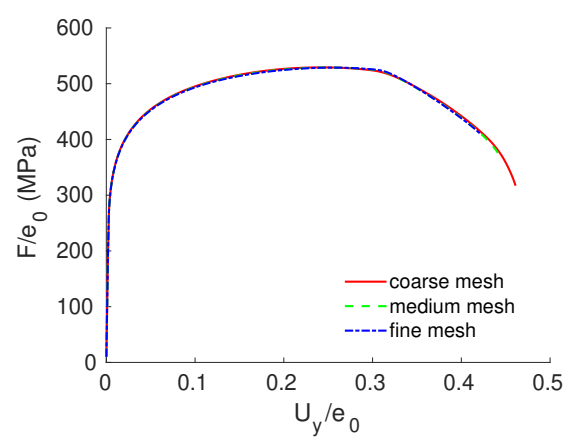

(b)

Figure 24: V-notched specimen - failure governed by void nucleation using a nucleated intensity $A_{n}=0.2$ and a sufficiently large $\xi$ in order not to trigger the shear coalescence: (a) with as sole nonlocality the volumetric plastic strain $\varepsilon_{\mathrm{v}}$, and (b) with the current framework with three nonlocal variables.

\subsubsection{Effect of model parameters}

The effects of the constitutive parameters on the shear-driven failure of the V-notched specimen are now studied. The effect of $\varepsilon_{\mathrm{d} s}$ is studied in Fig. 25(a). The result given by the GTN model considering $k_{\omega}=0$ is also reported for comparison purpose. When the value of $\varepsilon_{\mathrm{d} s}$ decreases while keeping $k_{\omega}=0$, an earlier onset of failure is observed, showing a similar effect as the one of $k_{\omega}$ in the GTN model enhanced with the original shear-growth model proposed by Nahshon and Hutchinson (2008). The effect of $k_{\omega}$ in the present model is studied in Figs. 25(b) and (c) for the two following cases:

i) The value $k_{\omega}$ increases while $\xi=1.015$ is kept constant: as a result of Eq. (111) the value of $\varepsilon_{\mathrm{d} s}$ decreases. The obtained results are depicted in Fig. 25(b) showing that $k_{\omega}$ has a similar effect as decreasing $\varepsilon_{\mathrm{d} s}$.

ii) The value $k_{\omega}$ increases while $\varepsilon_{\mathrm{d} s}=0.95$ is kept constant: as a result of Eq. (111) the value of $\xi$ increases. The obtained results are depicted in Fig. 25(c) showing that a larger value of $k_{w}$ leads to a later onset of failure, which is also observed in Fig. 8(c).

\subsection{Axisymmetric smooth bar in tension test}

This section investigates the failure of the axisymmetric smooth bar under uniaxial tensile loading. The geometry and loading conditions are reported in Fig. 26. The geometry parameters are chosen as $L=12.5 \mathrm{~mm}$ and $R_{0}=3 \mathrm{~mm}$. The finite element meshes presented in Figs. 11(a)-(d) are reconsidered in this section but with the axisymmetric 6 -node triangular elements.

Fig. 27(a) plots the force-radius reduction curves in terms of the nominal stress $\left(F /\left(\pi R_{0}^{2}\right)\right)$ versus the relative radius reduction $\left(-\Delta R / R_{0}\right)$ for the different meshes. In this case test, the stress triaxiality remains moderate and evolves within the necking region. The shear driven coalescence mode is preferred. The convergence with the mesh refinement is clearly observed for the medium and fine meshes. In the force-radius reduction curve, different stages can be distinguished: (i) a homogeneous elastoplastic stage with hardening until reaching maximum nominal stress; (ii) the necking stage in which the nominal stress starts to decrease; 


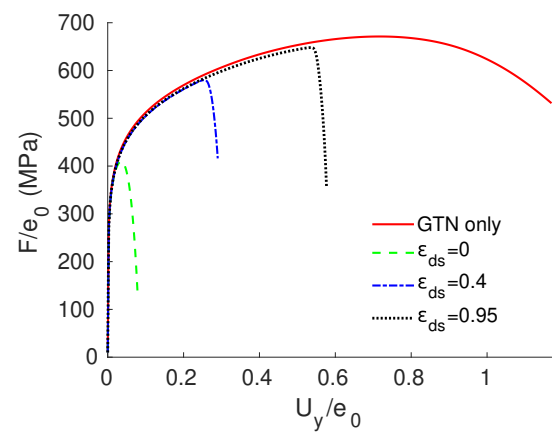

(a)

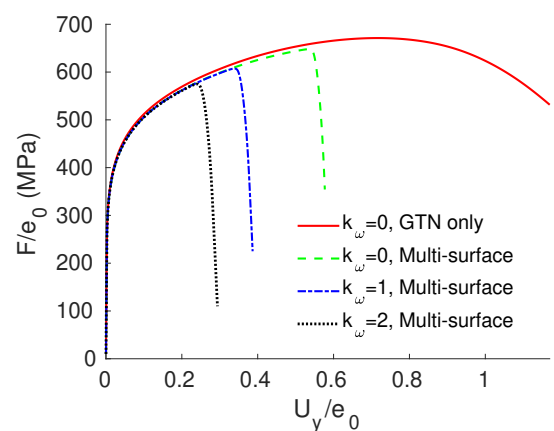

(b)

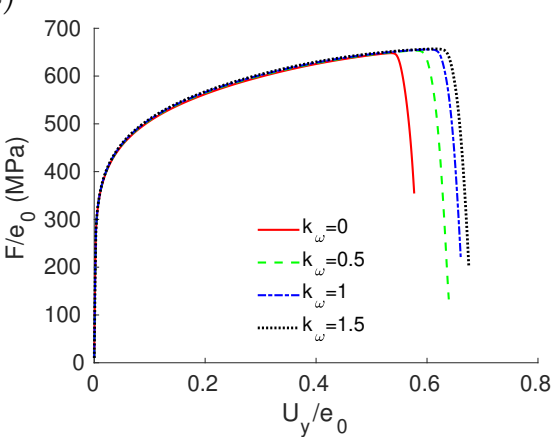

(c)

Figure 25: V-notched specimen: (a) influence of $\varepsilon_{\mathrm{d} s}$ for constant $k_{\omega}=0$, (b) influence of $k_{\omega}$ for constant $\xi=1.015$, and (c) influence of $k_{\omega}$ for constant $\varepsilon_{\mathrm{d} s}=0.95$.

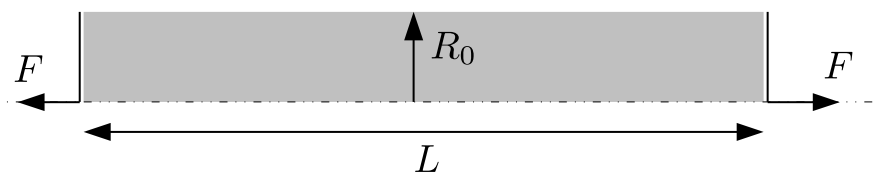

Figure 26: Axisymmetric smooth bar - geometry and boundary conditions. 


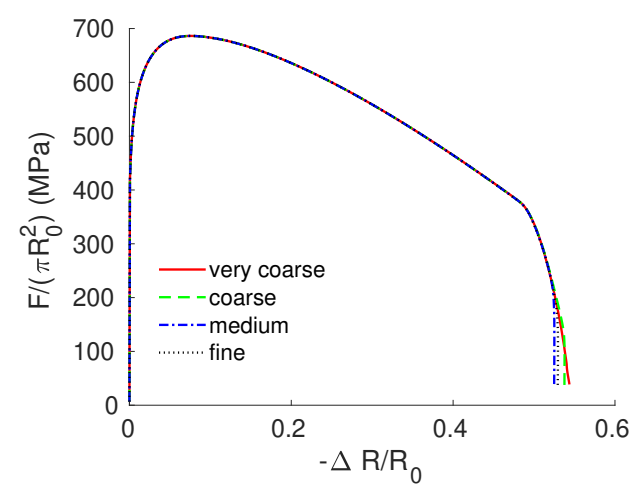

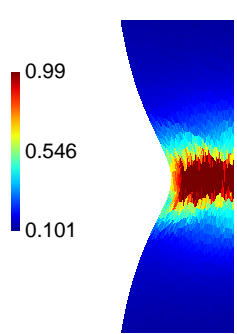

(b)

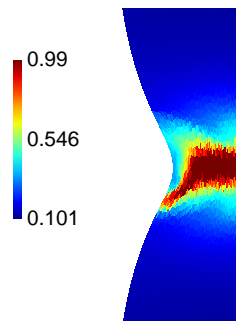

(c) (a)
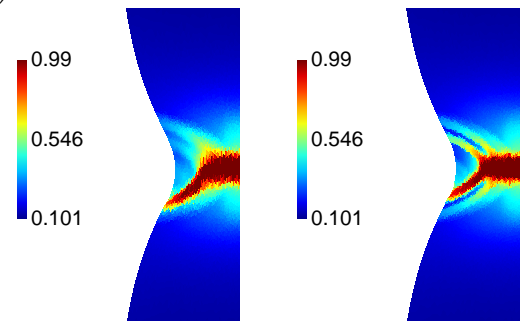

(d)

(e)

Figure 27: Axisymmetric smooth bar - mesh sensitivity: (a) force-radius reduction curves and distribution of the void ligament ratio $\chi$ at the last converged step with (b) the very coarse mesh, (c) the coarse mesh, (d) the medium mesh, and (e) the fine mesh.

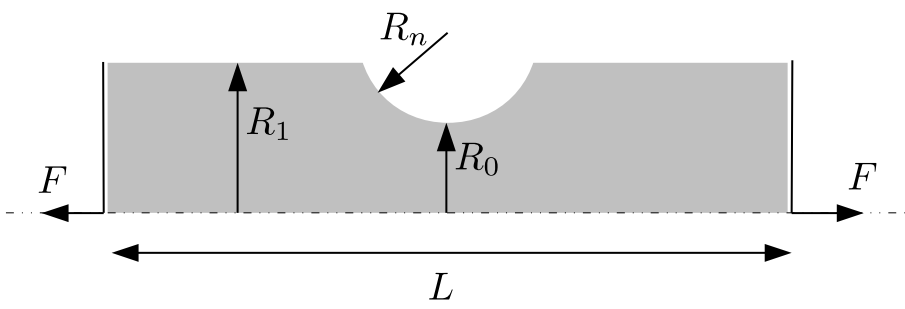

Figure 28: Axisymmetric notched bar: geometry and boundary conditions.

(iii) the nominal stress starting to drop faster corresponding to the onset of cracking stage in which a localization band perpendicular to the loading direction is formed, so-called cupfailure mode; (iv) the localization band propagating towards the free boundaries, a so-called cone-failure mode inclined at $45^{\circ}$ with the loading direction at the last failure stage and characterized by a suddenly drops of the load in the load-radius reduction curve. During these four stages, mesh convergence is observed for the medium and fine meshes because of the multi-nonlocal variable formulation. The cup-cone failure profiles for different finite element meshes are shown in Figs. 27(b)-(e) with the distribution of the void ligament ratio $\chi$ at the last converged step of each finite element mesh. Fig. 27(b) shows that the very coarse mesh cannot capture the cup-cone failure profile. 


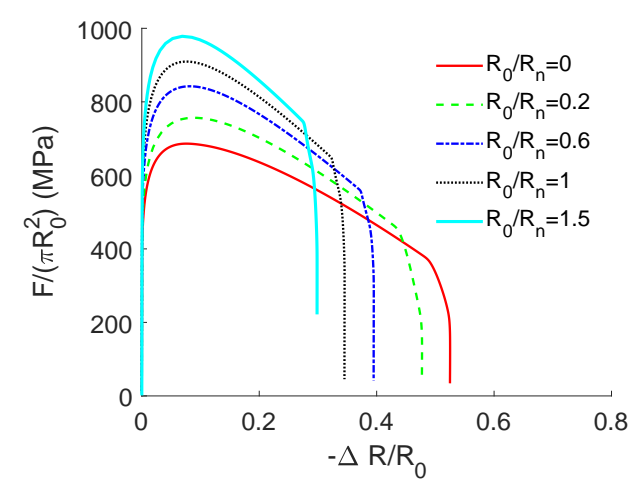

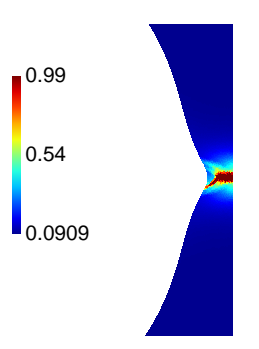

(b)

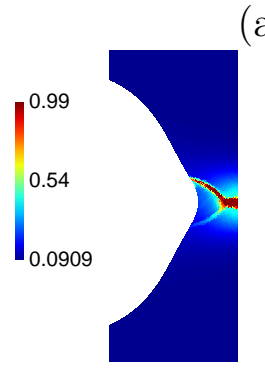

(c)

(a)

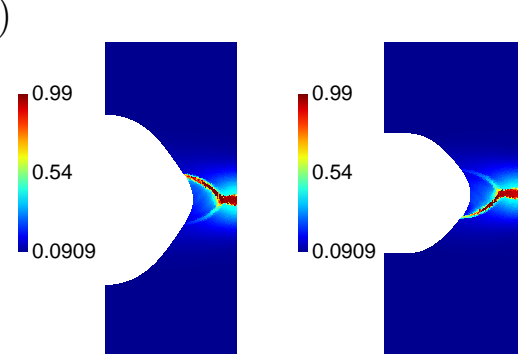

(e)

Figure 29: Axisymmetric notched bar - effect of the notch radius $R_{n}$ : (a) force-radius reduction curves and distribution of the void ligament ratio $\chi$ at the last converged step with (b) $R_{0} / R_{n}=0.2$, (c) $R_{0} / R_{n}=0.6$, (d) $R_{0} / R_{n}=1$, and (e) $R_{0} / R_{n}=1.5$.

\subsection{Ductile fracture of axisymmetric notched bars}

The plane strain specimen and the axisymmetric smooth bar under tension considered in the previous sections correspond to the cases with quite low to moderate stress triaxiality $(\eta<1$ during necking). The failure under higher stress triaxiality is considered in this section with axisymmetric notched bars under tensile loading conditions. The geometry and loading conditions are described in Fig. 28. The stress triaxiality at the center of the specimen at the notched section can be increased by decreasing the notch radius $R_{n}$ while $R_{0}$ remains constant. The smooth specimen corresponds to $R_{n}=\infty$. Fine enough meshes using axisymmetric 6-node triangular elements under-integrated by three Gauss points were chosen in order to ensure convergence upon mesh refinement.

Fig. 29(a) plots the force-radius reduction curves in terms of the nominal axial stress $\left(F /\left(\pi R_{0}^{2}\right)\right)$ versus the relative radius reduction at notched section $\left(-\Delta R / R_{0}\right)$ for different notch radii. The result corresponding to the smooth bar is also reported for comparison purpose. The ductility corresponds to the relative radius reduction at failure. The decrease of the ductility as a function of the notch radius is observed and agrees well with the experimental observations (Bao and Wierzbicki, 2004; Barsoum et al., 2012).

The failure pattern with different notch radius is shown in Figs. 29(b)-(e) as the distribution of the void ligament ratio $\chi$ at the last converged step corresponding to the last point in the force-radius reduction curve depicted in Fig. 29(a). A cup-cone failure profile is visible, demonstrating the ability of the model to capture this complex failure mode, which 
is sometimes observed in notched specimens.

\section{Conclusion}

An implicit gradient enhanced nonlocal framework is proposed to predict ductile failure in general loading conditions, involving conditions with extensive shear.

Three distinct nonlocal solutions for the expansion of voids embedded in an elastoplastic matrix are considered: (1) the void growth phase corresponding to the diffuse mode of plastic flow around the voids is modeled by the GTN yield surface as corrected by Nahshon and Hutchinson (2008) for shear; (2) the void coalescence mechanism corresponding to the localization of the plastic deformation between neighboring voids by internal necking is modeled by an extended version of the Thomason yield surface based on the maximum principal stress; and (3) the coalescence in shear is modeled by a yield surface based on the maximum shear stress. The three solutions are integrated in a multi-surface model, which is sensitive to both the stress triaxiality and the Lode variable. Under proportional loading conditions, the loading path dependence of the ductility defined by the deviatoric equivalent plastic strain at the onset of coalescence agrees with experimental observations by Bao and Wierzbicki (2004); Barsoum and Faleskog (2007); Barsoum et al. (2012); Faleskog and Barsoum (2013) for different loading states.

The implicit gradient enhanced nonlocal formulations are applied to multiple variables, leading to an efficient regularization of the loss of solution uniqueness when material softening occurs. A nonlocal effect is inactive if its corresponding nonlocal variable is not involved in the evolution laws of void characteristics. In this case, its corresponding nonlocal equation does not need to be integrated as it is separated from others with a trivial solution. Consequently, a nonlocal framework with 1,2 , or 3 nonlocal variables can be obtained that makes the current framework flexible. Since the multiple surface model involves different governing evolution laws, considering the nonlocality of the deviatoric and volumetric plastic equivalent strains is mandatory to obtain mesh-independent solution because of the porosity and void ligament ratio evolution, while considering the nonlocality of the mean equivalent plastic strain of the matrix is only required with the presence of void nucleation.

The constitutive relations are integrated in a fully implicit predictor-corrector scheme. The numerical framework was shown to be easily integrated into the conventional kinematicsbased finite element formulation as a result of the implicit nonlocal formulation. The capabilities of the proposed framework were studied through different numerical examples, showing that complex failure patterns such as slant and cup-cone of respectively plane strain and axisymmetric specimens under tensile loading conditions can be captured.

In future works, the material parameters calibration will be considered with a proper procedure followed by an experimental campaign. The evolution laws for void characteristics under the shear driven coalescence mode could be enhanced with a more complex micromechanics-based model. When the damage indicator is close to its critical value, the transition to crack will be considered by an energy consistent framework as considered by Wu et al. (2014); Leclerc et al. (2018, 2020). 


\section{Acknowledgments}

The research has been funded by the Walloon Region under the agreement no. 1610154EntroTough in the context of the 2016 Wallnnov call.

\section{Appendix A. Fully implicit integration of constitutive equations}

The numerical implementation of the porous plasticity model described by Eqs. (43-48) is detailed in this section. From the known kinematic inputs $\mathbf{F}, \overline{\mathbf{Z}}$ and internal variables, the unknown outputs $\mathbf{P}, \mathbf{Z}$, and their tangent operators have to be evaluated for the finite element resolution. Their computation follows a fully implicit predictor-corrector scheme during the time interval $\left[t_{n} t_{n+1}=t_{n}+\Delta t\right]$. For the sake of simplicity, all quantities at time $t_{n}$ are marked by a time index $n$ while the ones at time $t_{n+1}$ are specified without a time index.

\section{Appendix A.1. Elastic predictor}

The elastic predictor assumes a purely elastic state and the plastic part of the deformation gradient tensor is initialized from the one at the previous step, i.e. $\mathbf{F}^{\mathrm{ppr}}=\mathbf{F}_{n}^{\mathrm{p}}$. The predictor of the elastic part of the deformation gradient tensor is predicted using Eq. (2) by $\mathbf{F}^{\mathrm{epr}}=\mathbf{F} \cdot \mathbf{F}^{\mathrm{ppr}-1}$. The predictor of the elastic Cauchy strain tensor follows as $\mathbf{C}^{\mathrm{epr}}=$ $\mathbf{F}^{\mathrm{epr} T} \cdot \mathbf{F}^{\mathrm{epr}}=\mathbf{F}^{\mathrm{ppr}-T} \cdot \mathbf{F}^{T} \cdot \mathbf{F} \cdot \mathbf{F}^{\mathrm{ppr}-1}=\mathbf{F}^{\mathrm{ppr}-T} \cdot \mathbf{C} \cdot \mathbf{F}^{\mathrm{ppr}-1}$, from which the predictor of the elastic strain tensor is evaluated by $\mathbf{E}^{\mathrm{epr}}=\ln \sqrt{\mathbf{C}^{\mathrm{epr}}}$. From the value of $\mathbf{E}^{\mathrm{epr}}$, the predictor of the corotational Kirchhoff stress tensor $\boldsymbol{\tau}^{\text {pr }}$ is deduced using Eq. (39) as

$$
\tau^{\mathrm{pr}}=\mathbb{C}: \mathbf{E}^{\mathrm{epr}}
$$

The predictor of the corotational Cauchy stress is given by $\boldsymbol{\sigma}^{\mathrm{pr}}=J^{-1} \boldsymbol{\tau}^{\mathrm{pr}}$, where $J=\operatorname{det} \mathbf{F}$.

The evolution laws for $\mathbf{Y}$ are known following Eq. (47) and can be integrated in the time interval $\left[t_{n} t_{n+1}\right]$, leading to a general form ${ }^{7}$

$$
\mathbf{Y}^{\mathrm{pr}}=\mathbf{Y}_{n}+\Delta \mathbf{Y}\left(\Delta \overline{\mathbf{Z}}, \boldsymbol{\sigma}^{\mathrm{pr}}\right)
$$

where $\Delta \overline{\mathbf{Z}}=\overline{\mathbf{Z}}-\overline{\mathbf{Z}}_{n}$. The function $\Delta \mathbf{Y}$ is known and depends on the way the influence of the voids is accounted for, i.e. following the Gurson model or coalescence model.

The value of the yield surface $\Phi_{\mathrm{nl}}$ in Eq. (43) is estimated with all the relevant parameters. If the condition $\Phi_{\mathrm{nl}} \leq 0$ is satisfied, the elastic predictor is the solution and the final step described in Appendix A.3 is reached. Otherwise, the corrector step described in Appendix A.2 follows.

\footnotetext{
${ }^{7}$ The evolution laws of void characteristics are assumed to be integrated during the time interval $\left[t_{n} t_{n+1}=t_{n}+\Delta t\right]$ leading to an explicit dependence $\mathbf{Y}=\mathbf{Y}_{n}+\Delta \mathbf{Y}(\Delta \overline{\mathbf{Z}}, \boldsymbol{\sigma})$.
} 


\section{Appendix A.2. Plastic corrector}

Integrating Eq. (45) in the time interval $\left[t_{n} t_{n+1}\right]$ using an implicit approximation leads to the estimation of the current plastic part $\mathbf{F}^{\mathrm{p}}$ of the deformation gradient

$$
\mathbf{F}^{\mathrm{p}}=\exp \left(\Delta \mu \mathbf{N}^{\mathrm{p}}\right) \cdot \mathbf{F}^{\mathrm{ppr}}=\exp \left(\Delta \mathbf{E}^{p}\right) \cdot \mathbf{F}^{\mathrm{ppr}} .
$$

where $\Delta \mu=\mu-\mu_{n}, \mathbf{N}^{\mathrm{p}}$ is the normal of the plastic flow, which is estimated at the current stress state, and $\Delta \mathbf{E}^{p}=\Delta \mu \mathrm{N}^{\mathrm{p}}$ is the correction of the plastic deformation.

Using Eq. (A.3), the plastic problem described in Eqs. (43 - 48) results in the following finite incremental equations

$$
\begin{aligned}
& \boldsymbol{\tau}=\boldsymbol{\tau}^{\mathrm{pr}}-\mathbb{C}: \Delta \mathbf{E}^{p}, \\
& \boldsymbol{\sigma}=J^{-1} \boldsymbol{\tau}, \\
& \sigma_{\mathrm{Y}}=\sigma_{\mathrm{Y}}\left(\varepsilon_{\mathrm{m} n}+\Delta \varepsilon_{\mathrm{m}}\right), \\
& \mathbf{Y}=\mathbf{Y}_{n}+\Delta \mathbf{Y}(\Delta \overline{\mathbf{Z}}, \boldsymbol{\sigma}), \\
& \Phi_{\mathrm{nl}}\left(\boldsymbol{\sigma} ; \sigma_{\mathrm{Y}}, \mathbf{Y}\right)=0, \\
& \Delta \mathbf{E}^{p}-\Delta \mu \mathbf{N}^{\mathrm{p}}\left(\boldsymbol{\sigma} ; \sigma_{\mathrm{Y}}, \mathbf{Y}\right)=\mathbf{0}, \text { and } \\
& \boldsymbol{\sigma}: \Delta \mathbf{E}^{p}-(1-f) \sigma_{\mathrm{Y}} \Delta \varepsilon_{\mathrm{m}}=0 .
\end{aligned}
$$

Eqs. (A.4-A.10) can be iteratively solved for the unknowns $\boldsymbol{\tau}, \boldsymbol{\sigma}, \sigma_{\mathrm{Y}}, \Delta \varepsilon_{\mathrm{m}}, \mathbf{Y}, \Delta \mathbf{E}^{p}$, and $\Delta \mu$. Since Eqs. (A.4-A.7) can be eliminated from the other ones, the following nonlinear system of equations must finally be iteratively solved:

$$
\mathbf{r}=\left[\begin{array}{c}
r_{0} \\
\operatorname{vec}\left(\mathbf{r}_{1}\right) \\
r_{2}
\end{array}\right]=\left[\begin{array}{c}
\Phi_{\mathrm{nl}} \\
\operatorname{vec}\left(\Delta \mathbf{E}^{p}\right)-\Delta \mu \times \operatorname{vec}\left(\mathbf{N}^{\mathrm{p}}\right) \\
\frac{\boldsymbol{\sigma : \Delta \mathbf { E } ^ { p } - ( 1 - f ) \sigma _ { \mathrm { Y } } \Delta \varepsilon _ { \mathrm { m } }}}{\sigma_{\mathrm{Y}}^{0}}
\end{array}\right]=\mathbf{0}
$$

where $\Delta \mathbf{E}^{p}, \Delta \mu$, and $\Delta \varepsilon_{\mathrm{m}}$ are unknowns, $r_{0}, \mathbf{r}_{1}$ and $r_{2}$ denote the residuals of Eqs. (A.8A.10), $\sigma_{\mathrm{Y}}^{0}$ is used to obtain a dimensionless equation, and where vec $(\mathbf{A})$ is the vector representation of an arbitrary symmetric second order tensor $\mathbf{A}$ defined by

$$
\operatorname{vec}(\mathbf{A})=\left[\begin{array}{llllll}
A_{00} & A_{11} & A_{22} & A_{01} & A_{02} & A_{12}
\end{array}\right]^{T} .
$$

Since $\Delta \mathbf{E}^{p}$ is symmetric, one can define an unknowns vector

$$
\mathbf{X}=\left[\left[\begin{array}{lll}
\operatorname{vec}\left(\Delta \mathbf{E}^{p}\right) & ]^{T} \quad \Delta \mu & \Delta \varepsilon_{\mathrm{m}}
\end{array}\right]^{T} .\right.
$$

The nonlinear system of Eqs. (A.11) is iteratively solved using the Newton-Raphson procedure. For this purpose, the residual vector $\mathbf{r}$ is linearized at the current iteration as

$$
\mathbf{r}^{(k+1)}=\mathbf{r}^{(k)}+\mathbf{J}\left(\mathbf{X}^{(k+1)}-\mathbf{X}^{(k)}\right)=\mathbf{0}, \text { leading to } \mathbf{X}^{(k+1)}=\mathbf{X}^{(k)}-\mathbf{J}^{-1} \mathbf{r}^{(k)},
$$


where the Jacobian matrix $\mathbf{J}$ is given by

$$
\mathbf{J}=\frac{\partial \mathbf{r}}{\partial \mathbf{X}}=\left[\begin{array}{ccc}
{\left[\operatorname{vec}\left(\frac{\partial r_{0}}{\partial \Delta \mathbf{E}^{p}}\right)\right]^{T}} & \frac{\partial r_{0}}{\partial \Delta \mu} & \frac{\partial r_{0}}{\partial \Delta \varepsilon_{\mathrm{m}}} \\
\operatorname{mat}\left(\frac{\partial \mathbf{r}_{1}}{\partial \Delta \mathbf{E}^{p}}\right) & \operatorname{vec}\left(\frac{\partial \mathbf{r}_{1}}{\partial \Delta \mu}\right) & \operatorname{vec}\left(\frac{\partial \mathbf{r}_{1}}{\partial \Delta \varepsilon_{\mathrm{m}}}\right) \\
{\left[\operatorname{vec}\left(\frac{\partial r_{2}}{\partial \Delta \mathbf{E}^{p}}\right)\right]^{T}} & \frac{\partial r_{2}}{\partial \Delta \mu} & \frac{\partial r_{2}}{\partial \Delta \varepsilon_{\mathrm{m}}}
\end{array}\right],
$$

in which mat $(\mathbf{B})$ denotes the matrix representation of an arbitrary fourth order tensor $\mathbf{B}=\frac{\partial \mathbf{A}}{\partial \mathbf{C}}$ for two arbitrary symmetric second order tensors $\mathbf{A}$ and $\mathbf{C}$, see Appendix $\mathbf{B}$ for details. The derivatives of the residuals $r_{0}, \mathbf{r}_{1}$ and $r_{2}$ with respect to the unknowns $\Delta \mathbf{E}^{p}$, $\Delta \mu$, and $\Delta \varepsilon_{\mathrm{m}}$ at the iteration $k$ are detailed in Appendix $\mathrm{C}$.

Once the system of equations (A.11) is solved, the solution $\Delta \mathbf{E}^{p}$ allows estimating the values of $\mathbf{F}^{\mathrm{p}}$ using Eq. (A.3), and the increments of the deviatoric and volumetric plastic equivalent strains using Eqs. $(49,50)$ respectively as

$$
\begin{aligned}
\Delta \varepsilon_{\mathrm{d}} & =\sqrt{\frac{2}{3} \operatorname{dev}\left(\Delta \mathbf{E}^{p}\right): \operatorname{dev}\left(\Delta \mathbf{E}^{p}\right)}, \text { and } \\
\Delta \varepsilon_{\mathrm{v}} & =\operatorname{tr}\left(\Delta \mathbf{E}^{p}\right) .
\end{aligned}
$$

The local (internal) variables $\mathbf{Z}$ can be updated from their values at the previous time step following

$$
\left\{\begin{array}{l}
\varepsilon_{\mathrm{v}}=\varepsilon_{\mathrm{v} n}+\Delta \varepsilon_{\mathrm{v}} \\
\varepsilon_{\mathrm{m}}=\varepsilon_{\mathrm{m} n}+\Delta \varepsilon_{\mathrm{m}}, \text { and } \\
\varepsilon_{\mathrm{d}}=\varepsilon_{\mathrm{d} n}+\Delta \varepsilon_{\mathrm{d}}
\end{array}\right.
$$

Appendix A.3. Final step

The first Piola Kirchhoff stress $\mathbf{P}$ is eventually estimated using Eq. (26). However, the computation of the logarithmic operator used for estimating the elastic logarithmic strain $\mathbf{E}^{\mathrm{e}}=\ln \sqrt{\mathbf{C}^{\mathrm{e}}}$ potentially involves the following approximation

$$
\ln (\mathbf{A})=\mathbf{A}-\mathbf{I}-\frac{(\mathbf{A}-\mathbf{I})^{2}}{2}+\frac{(\mathbf{A}-\mathbf{I})^{3}}{3}+\ldots
$$

where $\mathbf{A}$ is an arbitrary second order tensor and $\mathbf{I}$ is the identity tensor, in which case, instead of using Eq. (26), $\mathbf{P}$ is deduced using

$$
\mathbf{P}=\mathbf{F}^{\mathrm{e}} \cdot(\boldsymbol{\tau}: \mathbf{L}) \cdot \mathbf{F}^{\mathrm{p}-T},
$$

where

$$
\mathbf{L}=\left.\frac{\partial \ln \mathbf{C}^{\mathrm{e}}}{\partial \mathbf{C}^{\mathrm{e}}}\right|_{\mathbf{C}^{\mathrm{e}}}
$$

The estimations of the material tangent operators $\frac{\partial \mathbf{P}}{\partial \mathbf{F}}, \frac{\partial \mathbf{P}}{\partial \mathbf{Z}}, \frac{\partial \mathbf{Z}}{\partial \mathbf{F}}, \frac{\partial \mathbf{Z}}{\partial \mathbf{Z}}$ required for the finite 
element iterative procedure are detailed in Appendix D.

\section{Appendix B. Matrix representation of a fourth order tensor}

The matrix representation of a fourth order tensor $\mathbf{B}=\frac{\partial \mathbf{A}}{\partial \mathbf{C}}$, where $\mathbf{A}$ and $\mathbf{C}$ are two symmetric second order tensors, can be defined as

$$
\begin{aligned}
\operatorname{mat}(\mathbf{B})= & \frac{\partial \operatorname{vec}(\mathbf{A})}{\partial \operatorname{vec}(\mathbf{C})} \\
& {\left[\begin{array}{llllll}
B_{0000} & B_{0011} & B_{0022} & B_{0001}+B_{0010} & B_{0002}+B_{0020} & B_{0012}+B_{0021} \\
B_{1100} & B_{1111} & B_{1122} & B_{1101}+B_{1110} & B_{1102}+B_{1120} & B_{1112}+B_{1121} \\
B_{2200} & B_{2211} & B_{2222} & B_{2201}+B_{2210} & B_{2202}+B_{2220} & B_{2212}+B_{2221} \\
B_{0100} & B_{0111} & B_{0122} & B_{0101}+B_{0110} & B_{0102}+B_{0120} & B_{0112}+B_{0121} \\
B_{0200} & B_{0211} & B_{0222} & B_{0201}+B_{0210} & B_{0202}+B_{0220} & B_{0212}+B_{0221} \\
B_{1200} & B_{1211} & B_{1222} & B_{1201}+B_{1210} & B_{1202}+B_{1220} & B_{1212}+B_{1221}
\end{array}\right] . }
\end{aligned}
$$

\section{Appendix C. Derivatives of the residuals $r_{0}, \mathrm{r}_{1}$ and $r_{2}$}

From the known forms of $\Phi_{\mathrm{nl}}$ and $\sigma_{\mathrm{Y}}$, one has

$$
\begin{aligned}
\frac{\partial r_{0}}{\partial \Delta \mathbf{E}^{p}} & =\left[\frac{\partial \Phi_{\mathrm{nl}}}{\partial \boldsymbol{\sigma}}+\left(\frac{\partial \Phi_{\mathrm{nl}}}{\partial \mathbf{Y}}\right)^{T} \frac{\partial \mathbf{Y}}{\partial \boldsymbol{\sigma}}\right]: \frac{\partial \boldsymbol{\sigma}}{\partial \Delta \mathbf{E}^{p}}, \\
\frac{\partial r_{0}}{\partial \Delta \mu} & =0 \\
\frac{\partial r_{0}}{\partial \Delta \varepsilon_{\mathrm{m}}} & =\frac{\partial \Phi_{\mathrm{nl}}}{\partial \sigma_{\mathrm{Y}}} H \\
\frac{\partial \mathbf{r}_{1}}{\partial \Delta \mathbf{E}^{p}} & =\mathcal{I}-\Delta \mu\left[\frac{\partial \mathbf{N}^{\mathrm{p}}}{\partial \boldsymbol{\sigma}}+\left(\frac{\partial \mathbf{N}^{\mathrm{p}}}{\partial \mathbf{Y}}\right)^{T} \frac{\partial \mathbf{Y}}{\partial \boldsymbol{\sigma}}\right]: \frac{\partial \boldsymbol{\sigma}}{\partial \Delta \mathbf{E}^{p}}, \\
\frac{\partial \mathbf{r}_{1}}{\partial \Delta \mu} & =-\mathbf{N}^{\mathrm{p}}, \\
\frac{\partial \mathbf{r}_{1}}{\partial \Delta \varepsilon_{\mathrm{m}}} & =-\Delta \mu \frac{\partial \mathbf{N}^{\mathrm{p}}}{\partial \sigma_{\mathrm{Y}}}, \\
\frac{\partial r_{2}}{\partial \Delta \mathbf{E}^{p}} & =\frac{1}{\sigma_{\mathrm{Y}}^{0}}\left[\left(\Delta \mathbf{E}^{p}+\sigma_{\mathrm{Y}} \Delta \varepsilon_{\mathrm{m}} \frac{\partial f}{\partial \boldsymbol{\sigma}}\right): \frac{\partial \boldsymbol{\sigma}}{\partial \Delta \mathbf{E}^{p}}+\boldsymbol{\sigma}\right] \\
\frac{\partial r_{2}}{\partial \Delta \mu} & =0, \text { and } \\
\frac{\partial r_{2}}{\partial \Delta \varepsilon_{\mathrm{m}}} & =-\frac{1}{\sigma_{\mathrm{Y}}^{0}}(1-f)\left(\sigma_{\mathrm{Y}}+H \Delta \varepsilon_{\mathrm{m}}\right),
\end{aligned}
$$

where $H=\frac{\partial \sigma_{\mathrm{Y}}}{\partial \varepsilon_{\mathrm{m}}}$ is the plastic hardening modulus of the matrix material. 


\section{Appendix D. Material tangent operators}

The material tangent operators $\frac{\partial \mathbf{P}}{\partial \mathbf{F}}, \frac{\partial \mathbf{P}}{\partial \overline{\mathbf{Z}}}, \frac{\partial \mathbf{Z}}{\partial \mathbf{F}}$, and $\frac{\partial \mathbf{Z}}{\partial \overline{\mathbf{Z}}}$ required for the iterative procedure of the finite element resolution must be estimated. With an arbitrary quantity •, one always has

$$
\begin{aligned}
\frac{\partial \bullet}{\partial \mathbf{F}} & =\frac{\partial \bullet}{\partial \mathbf{C}}: \frac{\partial \mathbf{C}}{\partial \mathbf{F}}=2 \mathbf{F} \cdot \frac{\partial \bullet}{\partial \mathbf{C}}, \\
\frac{\partial \bullet}{\partial \mathbf{C}} & =\mathbf{F}^{\mathrm{ppr}-1} \cdot \frac{\partial \bullet}{\partial \mathbf{C}^{\mathrm{epr}}} \cdot \mathbf{F}^{\mathrm{ppr}-T}, \text { and } \\
\frac{\partial \bullet}{\partial \mathbf{C}^{\mathrm{epr}}} & =\frac{\partial \bullet}{\partial \mathbf{E}^{\mathrm{epr}}}: \frac{1}{2} \mathbf{L}^{\mathrm{pr}},
\end{aligned}
$$

leading to

$$
\frac{\partial \bullet}{\partial \mathbf{F}}=\frac{\partial \bullet}{\partial \mathbf{E}^{\mathrm{epr}}}:\left[\mathbf{L}^{\mathrm{pr}}\left[:^{2,4}\right]\left(\mathbf{F}^{\mathrm{epr}} \otimes \mathbf{F}^{\mathrm{ppr}-1}\right)\right]
$$

where $[: i, j]$ is the double dot tensor operator considering the $i^{\text {th }}$ and $j^{\text {th }}$ indexes of the right tensor. As a consequence, in order to estimate the derivatives with respect to $\mathbf{F}$, the ones with respect to $\mathbf{E}^{\mathrm{epr}}$ need to be estimated.

Since the first Piola-Kirchhoff stress is estimated by Eq. (A.20), in order to evaluate $\frac{\partial \mathbf{P}}{\partial \mathbf{F}}$, and $\frac{\partial \mathbf{P}}{\partial \mathbf{Z}}$, the terms $\frac{\partial \mathbf{F}^{\mathrm{p}}}{\partial \mathbf{F}}, \frac{\partial \mathbf{F}^{\mathrm{p}}}{\partial \mathbf{Z}}, \frac{\partial \tau}{\partial \mathbf{F}}$, and $\frac{\partial \tau}{\partial \mathbf{Z}}$ need to be estimated, and the missing derivations read

$$
\begin{aligned}
\frac{\partial \mathbf{F}^{\mathrm{p}-1}}{\partial \mathbf{F}} & =-\mathbf{F}^{\mathrm{p}-1} \cdot\left(\frac{\partial \mathbf{F}^{\mathrm{p}}}{\partial \mathbf{F}}\right)\left[^{2} \cdot\right] \mathbf{F}^{\mathrm{p}-1}, \\
\frac{\partial \mathbf{F}^{\mathrm{e}}}{\partial \mathbf{F}} & \left.=\frac{\partial\left(\mathbf{F} \cdot \mathbf{F}^{\mathrm{p}-1}\right)}{\partial \mathbf{F}}=\mathbb{I}^{2} \cdot\right] \mathbf{F}^{\mathrm{p}-1}+\mathbf{F} \cdot \frac{\partial \mathbf{F}^{\mathrm{p}-1}}{\partial \mathbf{F}}, \\
\frac{\partial \mathbf{L}}{\partial \mathbf{F}} & =\frac{\partial \mathbf{L}}{\partial \mathbf{C}^{\mathrm{e}}}: \frac{\partial \mathbf{C}^{\mathrm{e}}}{\partial \mathbf{F}^{\mathrm{e}}}: \frac{\partial \mathbf{F}^{\mathrm{e}}}{\partial \mathbf{F}}=\mathbf{H}:\left(2 \mathbf{F}_{e}^{T} \cdot \frac{\partial \mathbf{F}_{e}}{\partial \mathbf{F}}\right), \\
\frac{\partial \mathbf{F}^{\mathrm{p}-1}}{\partial \bar{Z}_{k}} & =-\mathbf{F}^{\mathrm{p}-1} \cdot \frac{\partial \mathbf{F}^{\mathrm{p}}}{\partial \bar{Z}_{k}} \cdot \mathbf{F}^{\mathrm{p}-1}, \\
\frac{\partial \mathbf{F}^{\mathrm{e}}}{\partial \bar{Z}_{k}} & =\frac{\partial\left(\mathbf{F} \cdot \mathbf{F}^{\mathrm{p}-1}\right)}{\partial \bar{Z}_{k}}=\mathbf{F} \cdot \frac{\partial \mathbf{F}^{\mathrm{p}-1}}{\partial \bar{Z}_{k}}, \text { and } \\
\frac{\partial \mathbf{L}}{\partial \bar{Z}_{k}} & =\frac{\partial \mathbf{L}}{\partial \mathbf{C}^{\mathrm{e}}}: \frac{\partial \mathbf{C}^{\mathrm{e}}}{\partial \mathbf{F}^{\mathrm{e}}}: \frac{\partial \mathbf{F}^{\mathrm{e}}}{\partial \bar{Z}_{k}}=\mathbf{H}:\left(2 \mathbf{F}^{\mathrm{e} T} \cdot \frac{\partial \mathbf{F}^{\mathrm{e}}}{\partial \bar{Z}_{k}}\right),
\end{aligned}
$$

where $\mathbb{I}_{i j k l}=\delta_{i k} \delta_{j l}$ and $\mathbf{H}=\frac{\partial \mathbf{L}}{\partial \mathbf{C}^{\mathrm{e}}}$, and where $\left[{ }^{i} \cdot\right]$ is the dot tensor operator considering the $i^{t h}$ index of the left tensor. In order to evaluate $\frac{\partial \mathbf{F}^{\mathrm{p}}}{\partial \mathbf{F}}$ and $\frac{\partial \boldsymbol{\tau}}{\partial \mathbf{F}}$, Eq. (D.4) is used. The last missing terms are thus $\frac{\partial \mathbf{F}^{\mathrm{p}}}{\partial \mathbf{E}^{\mathrm{epr}}}, \frac{\partial \mathbf{F}^{\mathrm{p}}}{\partial \overline{\mathbf{Z}}}, \frac{\partial \boldsymbol{\tau}}{\partial \mathbf{E}^{\mathrm{epr}}}$, and $\frac{\partial \boldsymbol{\tau}}{\partial \mathbf{Z}}$. The consistency of Eq. (A.11) is considered, leading to the following relation

$$
\dot{\mathbf{r}}=\mathbf{J} \dot{\mathbf{X}}+\frac{\partial \mathbf{r}}{\partial \mathbf{E}^{\mathrm{epr}}}: \dot{\mathbf{E}}^{\mathrm{epr}}+\frac{\partial \mathbf{r}}{\partial \overline{\mathbf{Z}}} \dot{\overline{\mathbf{Z}}}=\mathbf{0}
$$


The last equation allows estimating

$$
\frac{\partial \mathbf{X}}{\partial \mathbf{E}^{\mathrm{epr}}}=-\mathbf{J}^{-1} \frac{\partial \mathbf{r}}{\partial \mathbf{E}^{\mathrm{epr}}}, \text { and } \frac{\partial \mathbf{X}}{\partial \overline{\mathbf{Z}}}=-\mathbf{J}^{-1} \frac{\partial \mathbf{r}}{\partial \overline{\mathbf{Z}}},
$$

where $\frac{\partial \mathbf{r}}{\partial \mathbf{E}^{\text {epr }}}$ and $\frac{\partial \mathbf{r}}{\partial \overline{\mathbf{Z}}}$ are given as

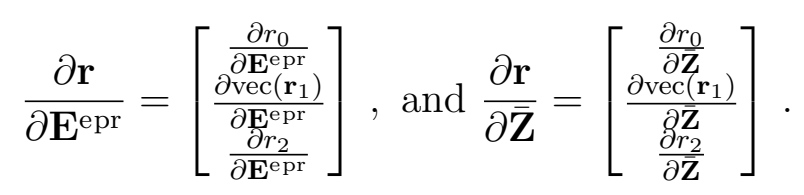

Once the explicit forms of $\Phi_{\mathrm{nl}}\left(\boldsymbol{\sigma} ; \sigma_{\mathrm{Y}}, \mathbf{Y}\right), \mathbf{N}^{\mathrm{p}}\left(\boldsymbol{\sigma} ; \sigma_{\mathrm{Y}}, \mathbf{Y}\right), \sigma_{\mathrm{Y}}\left(\varepsilon_{\mathrm{m}}\right)$, and $\Delta \mathbf{Y}(\Delta \overline{\mathbf{Z}}, \boldsymbol{\sigma})$ are known, one can estimate $\frac{\partial \mathbf{r}}{\partial \mathbf{E}^{\text {epr }}}$ and $\frac{\partial \mathbf{r}}{\partial \overline{\mathbf{Z}}}$ from

$$
\begin{aligned}
\frac{\partial r_{0}}{\partial \mathbf{E}^{\mathrm{epr}}} & =\left[\frac{\partial \Phi_{\mathrm{nl}}}{\partial \boldsymbol{\sigma}}+\left(\frac{\partial \Phi_{\mathrm{nl}}}{\partial \mathbf{Y}}\right)^{T} \frac{\partial \mathbf{Y}}{\partial \boldsymbol{\sigma}}\right]: \frac{\partial \boldsymbol{\sigma}}{\partial \mathbf{E}^{\mathrm{epr}}} \\
\frac{\partial \mathbf{r}_{1}}{\partial \mathbf{E}^{\mathrm{epr}}} & =-\Delta \mu\left[\frac{\partial \mathbf{N}^{\mathrm{p}}}{\partial \boldsymbol{\sigma}}+\left(\frac{\partial \mathbf{N}^{\mathrm{p}}}{\partial \mathbf{Y}}\right)^{T} \frac{\partial \mathbf{Y}}{\partial \boldsymbol{\sigma}}\right]: \frac{\partial \boldsymbol{\sigma}}{\partial \mathbf{E}^{\mathrm{epr}}}, \\
\frac{\partial r_{2}}{\partial \mathbf{E}^{\mathrm{epr}}} & =\frac{1}{\sigma_{\mathrm{Y}}^{0}}\left(\Delta \mathbf{E}^{p}+\sigma_{\mathrm{Y}} \Delta \varepsilon_{\mathrm{m}} \frac{\partial f}{\partial \boldsymbol{\sigma}}\right): \frac{\partial \boldsymbol{\sigma}}{\partial \mathbf{E}^{\mathrm{epr}}} \\
\frac{\partial r_{0}}{\partial \overline{\mathbf{Z}}} & =\left(\frac{\partial \Phi_{\mathrm{nl}}}{\partial \mathbf{Y}}\right)^{T} \frac{\partial \mathbf{Y}}{\partial \overline{\mathbf{Z}}}, \\
\frac{\partial \mathbf{r}_{1}}{\partial \overline{\mathbf{Z}}} & =-\Delta \mu\left(\frac{\partial \mathbf{N}^{\mathrm{p}}}{\partial \mathbf{Y}}\right)^{T} \frac{\partial \mathbf{Y}}{\partial \overline{\mathbf{Z}}}, \text { and } \\
\frac{\partial r_{2}}{\partial \overline{\mathbf{Z}}} & =\frac{\sigma_{\mathrm{Y}} \Delta \varepsilon_{\mathrm{m}}}{\sigma_{\mathrm{Y}}^{0}} \frac{f}{\partial \overline{\mathbf{Z}}} \cdot
\end{aligned}
$$

Using the estimation of $\frac{\partial \mathbf{X}}{\partial \mathbf{E}^{\mathrm{epr}}}$ and $\frac{\partial \mathbf{X}}{\partial \overline{\mathbf{Z}}}$ from Eqs. (D.12), $\frac{\partial \Delta \mathbf{E}^{p}}{\partial \mathbf{E}^{\mathrm{epr}}}, \frac{\partial \Delta \mathbf{E}^{p}}{\partial \overline{\mathbf{Z}}}, \frac{\partial \Delta \varepsilon_{\mathrm{m}}}{\partial \mathbf{E}^{\mathrm{epr}}}, \frac{\partial \Delta \varepsilon_{\mathrm{m}}}{\partial \mathbf{Z}}$ are known and Eqs. (A.1, A.4) thus lead to

$$
\begin{aligned}
\frac{\partial \boldsymbol{\tau}}{\partial \mathbf{E}^{\mathrm{epr}}} & =\frac{\partial \boldsymbol{\tau}^{\mathrm{pr}}}{\partial \mathbf{E}^{\mathrm{epr}}}-\mathbb{C}: \frac{\partial \Delta \mathbf{E}^{p}}{\partial \mathbf{E}^{\mathrm{epr}}}=\mathbb{C}-\mathbb{C}: \frac{\partial \Delta \mathbf{E}^{p}}{\partial \mathbf{E}^{\mathrm{epr}}}, \text { and } \\
\frac{\partial \boldsymbol{\tau}}{\partial \bar{Z}_{k}} & =-\mathbb{C}: \frac{\partial \Delta \mathbf{E}^{p}}{\partial \bar{Z}_{k}} .
\end{aligned}
$$

Because the operator exp used in Eq. (A.3) is, in general, approximated by

$$
\exp (\mathbf{A})=\mathbf{I}+\mathbf{A}+\frac{\mathbf{A}^{2}}{2 !}+\frac{\mathbf{A}^{3}}{3 !}+\ldots,
$$


where $\mathbf{A}$ is an arbitrary second order tensor, Eq. (A.3) results in

$$
\begin{aligned}
\frac{\partial \mathbf{F}^{\mathrm{p}}}{\partial \mathbf{E}^{\mathrm{epr}}} & =\left(\mathcal{E}: \frac{\partial \Delta \mathbf{E}^{p}}{\partial \mathbf{E}^{\mathrm{epr}}}\right)\left[{ }^{2} \cdot\right] \mathbf{F}^{\mathrm{ppr}}, \text { and } \\
\frac{\partial \mathbf{F}^{\mathrm{p}}}{\partial \bar{Z}_{k}} & =\left(\mathcal{E}: \frac{\partial \Delta \mathbf{E}^{p}}{\partial \bar{Z}_{k}}\right)\left[{ }^{2} \cdot\right] \mathbf{F}^{\mathrm{ppr}},
\end{aligned}
$$

where $\mathcal{E}=\frac{\partial \exp \Delta \mathbf{E}^{p}}{\partial \Delta \mathbf{E}^{p}}$.

Finally, the terms $\frac{\partial \mathbf{Z}}{\partial \mathbf{F}}$ and $\frac{\partial \mathbf{Z}}{\partial \overline{\mathbf{Z}}}$ have to be computed. Since $\frac{\partial \mathbf{Z}}{\partial \mathbf{F}}$ is deduced from $\frac{\partial \mathbf{Z}}{\partial \mathbf{E}^{\text {epr }}}$ using Eq. (D.4), from the definition of the local variables following Eq. (51), one computes

$$
\begin{aligned}
\frac{\partial \mathbf{Z}}{\partial \mathbf{E}^{\mathrm{epr}}} & =\left[\begin{array}{lll}
\frac{\partial \Delta \varepsilon_{\mathrm{v}}}{\partial \mathbf{E}^{\mathrm{ep}}} & \frac{\partial \Delta \varepsilon_{\mathrm{m}}}{\partial \mathbf{E}^{\mathrm{epr}}} & \frac{\partial \Delta \varepsilon_{\mathrm{d}}}{\partial \mathbf{E}^{\mathrm{epr}}}
\end{array}\right]^{T}, \text { and } \\
\frac{\partial \mathbf{Z}}{\partial \overline{\mathbf{Z}}} & =\left[\begin{array}{lll}
\frac{\partial \Delta \varepsilon_{\mathrm{v}}}{\partial \overline{\mathbf{Z}}} & \frac{\partial \Delta \varepsilon_{\mathrm{m}}}{\partial \overline{\mathbf{Z}}} & \frac{\partial \Delta \varepsilon_{\mathrm{d}}}{\partial \overline{\mathbf{Z}}}
\end{array}\right]^{T},
\end{aligned}
$$

where, using Eqs. (A.16, A.17),

$$
\frac{\partial \Delta \varepsilon_{\mathrm{v}}}{\partial \bullet}=\mathbf{I}: \frac{\partial \Delta \mathbf{E}^{p}}{\partial \bullet}, \text { and } \frac{\partial \Delta \varepsilon_{\mathrm{d}}}{\partial \bullet}=\frac{2}{3 \Delta \varepsilon_{\mathrm{d}}} \operatorname{dev}\left(\Delta \mathbf{E}^{p}\right): \frac{\partial \Delta \mathbf{E}^{p}}{\partial \bullet}
$$

in which $\bullet$ represents either $\mathbf{E}^{\mathrm{epr}}$ or $\overline{\mathbf{Z}}$.

\section{Appendix E. Smooth regularization of yield functions}

Appendix E.1. Smooth MPS-based Thomason yield surface

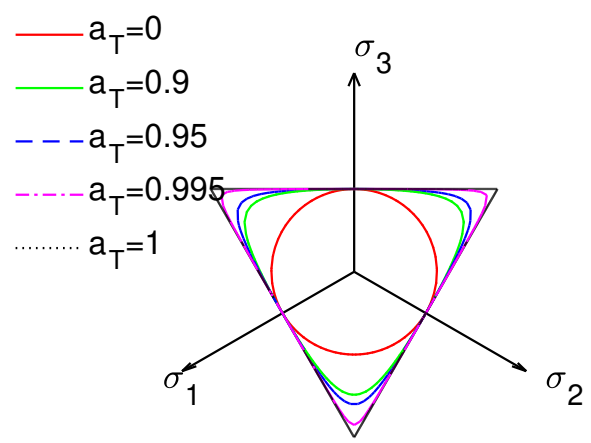

(a)

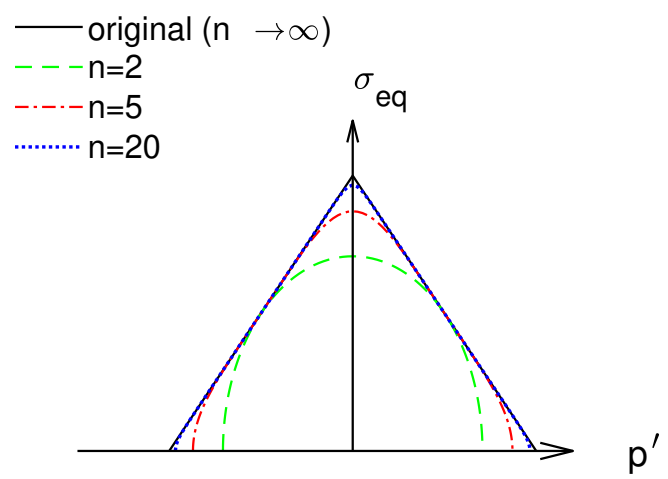

(b)

Figure E.30: Smooth MPS-based Thomason yield surface: (a) in $\pi$-plane (under a constant pressure) and (b) in $\theta$-plane (under a constant $\theta$ ). The original model (75) is obtained with $a_{T}=1$ and $n=\infty$.

With the use of the Thomason effective stress following Eq. (76), the MPS-based Thomason yield surface (75) has singularities at $p^{\prime}=0$ (under pure shear), at $\sigma_{\text {eq }}=0$ (under hydrostatic pressure), and at $\theta=\frac{\pi}{3}$ (at the stress state $\sigma_{1}=\sigma_{2}>\sigma_{3}$ ), see Fig. E.30. These 
corners are rounded in order to produce a smooth normal vector all along the yield surface as follows:

- The corners at $\theta=\frac{\pi}{3}$ are rounded by replacing $\theta$ in Eq. (76) by $\theta_{T}$ where

$$
\theta_{T}=\frac{1}{3} \arccos \left[1+a_{T}(\omega-1)\right]
$$

in which $a_{T} \in\left[\begin{array}{ll}0 & 1\end{array}\right]$ is a user parameter. Therefore the Thomason effective stress (76) is first modified as

$$
\hat{\sigma}_{\mathrm{T}}=\frac{1}{C_{\mathrm{Tf}}}\left(\frac{2}{3} \sigma_{\mathrm{eq}} \cos \theta_{T}+\left|p^{\prime}\right|\right) .
$$

The influence of $a_{T}$ on the shape of the MPS-based Thomason yield surface is shown in Fig. E.30(a). The original model described by Eq. (72) is recovered with $a_{T}=1$. The Thomason model considered in the works of Benzerga et al. (2002); Besson (2009); Benzerga and Leblond (2010); Pardoen and Hutchinson (2000, 2003); Pardoen (2006) corresponds to $a_{T}=0$. The value of $a_{T} \lesssim 1$ should be chosen in order to obtain a smooth version close to the original one. In this work $a_{T}=0.995$ is used.

- The corners at $p^{\prime}=0$ and at $\sigma_{\text {eq }}=0$ are rounded using the methodology proposed by Besson (2009). As a result, the Thomason effective stress described in Eq. (E.2) is modified as

$$
\hat{\sigma}_{\mathrm{T}}=\frac{1}{C_{\mathrm{Tf}}}\left(\left|\frac{2}{3} \sigma_{\mathrm{eq}} \cos \theta_{T}+p^{\prime}\right|^{n}+\left|\frac{2}{3} \sigma_{\mathrm{eq}} \cos \theta_{T}-p^{\prime}\right|^{n}\right)^{\frac{1}{n}}
$$

where $n$ is a user defined parameter. A value of $n \gg 1$ should be chosen in order to get a smooth version close to the original one. The influence of $n$ on the shape of the MPS-based Thomason yield surface is shown in Fig. E.30(b). Clearly, a value $n \geq 20$ allows remaining very close to the original model. In this work $n=50$ is considered.

\section{Appendix E.2. Smooth MSS-based yield surface}

With the use of the shear effective stress in Eq. (85), the MSS-based yield condition (84) has singularities at $\theta=0$ and $\theta=\frac{\pi}{3}$ respectively corresponding to $\omega=1$ and $\omega=-1$. These singularities are removed by replacing $\theta$ in Eq. (85) by $\theta_{S}$ where

$$
\theta_{S}=\frac{1}{3} \arccos \left(a_{S} \omega\right)
$$

in which $a_{S} \in\left[\begin{array}{ll}0 & 1\end{array}\right]$ is a user defined parameter. As a result, the shear effective stress (85) is modified as follows

$$
\hat{\sigma}_{\mathrm{S}}=\frac{\sigma_{\mathrm{eq}}}{C_{\mathrm{Sf}}}\left(\frac{\sin \theta_{S}}{2}+\frac{\sqrt{3} \cos \theta_{S}}{2}\right) .
$$




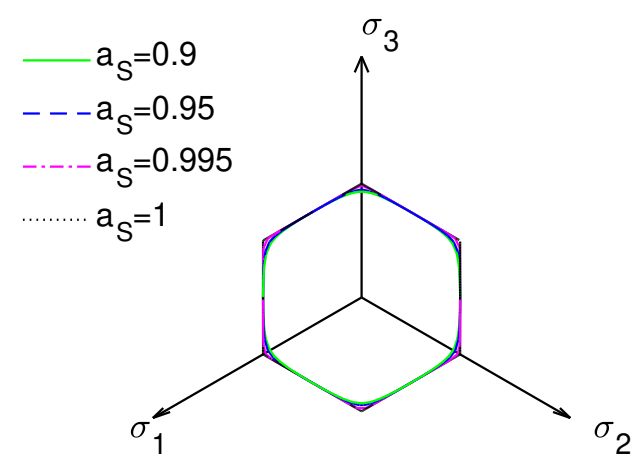

Figure E.31: Smooth MSS-based yield surface in $\pi$-plane (under a constant pressure).

The influence of $a_{S}$ on the shape of the MSS-based yield surface is shown in Fig. E.31. The unsmoothed model described by Eq. (72) is recovered with $a_{S}=1$. The value of $a_{S} \lesssim 1$ should be chosen in order to obtain a smooth version close to the original one. In this work $a_{S}=0.995$ is used.

\section{Appendix F. Derivatives of yield surface and plastic flow normal}

Appendix F.1. Some useful tensorial derivatives

For an arbitrary tensor $\boldsymbol{\sigma}$, whose three invariants are

$$
p^{\prime}=\frac{\operatorname{tr}(\boldsymbol{\sigma})}{3}, \sigma_{\mathrm{eq}}=\sqrt{\frac{3}{2} \operatorname{dev}(\boldsymbol{\sigma}): \operatorname{dev}(\boldsymbol{\sigma})}, \text { and } J_{3}=\operatorname{det} \operatorname{dev}(\boldsymbol{\sigma}) \text {, }
$$

one always has

$$
\begin{aligned}
\frac{\partial p^{\prime}}{\partial \boldsymbol{\sigma}} & =\frac{\mathbf{I}}{3}, \\
\frac{\partial \sigma_{\mathrm{eq}}}{\partial \boldsymbol{\sigma}} & =\frac{3 \operatorname{dev}(\boldsymbol{\sigma})}{2 \sigma_{\mathrm{eq}}} \\
\frac{\partial J_{3}}{\partial \boldsymbol{\sigma}} & =\operatorname{dev}(\boldsymbol{\sigma}) \cdot \operatorname{dev}(\boldsymbol{\sigma})-\frac{2 \sigma_{\mathrm{eq}}^{2}}{9} \mathbf{I}, \\
\frac{\partial^{2} \sigma_{\mathrm{eq}}}{\partial \boldsymbol{\sigma} \otimes \partial \boldsymbol{\sigma}} & =\frac{3 \mathcal{I}^{\operatorname{dev}}}{2 \sigma_{\mathrm{eq}}}-\frac{3 \operatorname{dev}(\boldsymbol{\sigma})}{2 \sigma_{\mathrm{eq}}^{2}} \otimes \frac{\partial \sigma_{\mathrm{eq}}}{\partial \boldsymbol{\sigma}}, \text { and } \\
\frac{\partial^{2} J_{3}}{\partial \boldsymbol{\sigma} \otimes \partial \boldsymbol{\sigma}} & =\mathcal{I}^{\operatorname{dev}}[2 \cdot] \operatorname{dev}(\boldsymbol{\sigma})+\operatorname{dev}(\boldsymbol{\sigma}) \cdot \mathcal{I}^{\operatorname{dev}}-\frac{2}{3} \mathbf{I} \otimes \operatorname{dev}(\boldsymbol{\sigma}) .
\end{aligned}
$$




\section{Appendix F.2. GTN yield function}

The derivatives of the GTN yield function following Eq. (53) with respect to $\boldsymbol{\sigma}, \sigma_{\mathrm{Y}}$, and $\mathbf{Y}$ are given as follows

$$
\begin{aligned}
& \frac{\partial \Phi_{\mathrm{G}}}{\partial \boldsymbol{\sigma}}=\frac{1}{\sigma_{\mathrm{Y}}} \frac{\partial \hat{\sigma}_{\mathrm{G}}}{\partial u} \frac{\partial u}{\partial \boldsymbol{\sigma}}, \\
& \frac{\partial \Phi_{\mathrm{G}}}{\partial \sigma_{\mathrm{Y}}}=-\frac{\hat{\sigma}_{\mathrm{G}}}{\sigma_{\mathrm{Y}}^{2}}+\frac{1}{\sigma_{\mathrm{Y}}} \frac{\partial \hat{\sigma}_{\mathrm{G}}}{\partial u} \frac{\partial u}{\partial \sigma_{\mathrm{Y}}} \text {, and }
\end{aligned}
$$

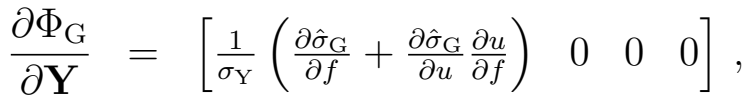

where, following Eq. (54), $\hat{\sigma}_{\mathrm{G}}$ can be rewritten as

$$
\hat{\sigma}_{\mathrm{G}}=\frac{\sqrt{u}}{1-q_{1} f}, \text { with } u=\sigma_{\mathrm{eq}}^{2}+2 \sigma_{\mathrm{Y}}^{2} f q_{1}\left[\cosh \left(\frac{3}{2} q_{2} \frac{p^{\prime}}{\sigma_{\mathrm{Y}}}\right)-1\right] \text {, }
$$

and

$$
\begin{aligned}
\frac{\partial \hat{\sigma}_{\mathrm{G}}}{\partial u} & =\frac{1}{2 \sqrt{u}\left(1-q_{1} f\right)}, \\
\frac{\partial u}{\partial \boldsymbol{\sigma}} & =3 \operatorname{dev}(\boldsymbol{\sigma})+\sigma_{\mathrm{Y}} f q_{1} q_{2} \sinh \left(\frac{3}{2} q_{2} \frac{p^{\prime}}{\sigma_{\mathrm{Y}}}\right) \mathbf{I}, \\
\frac{\partial u}{\partial \sigma_{\mathrm{Y}}} & =4 \sigma_{\mathrm{Y}} f q_{1}\left[\cosh \left(\frac{3}{2} q_{2} \frac{p^{\prime}}{\sigma_{\mathrm{Y}}}\right)-1\right]-3 p^{\prime} f q_{1} q_{2} \sinh \left(\frac{3}{2} q_{2} \frac{p^{\prime}}{\sigma_{\mathrm{Y}}}\right), \\
\frac{\partial \hat{\sigma}_{\mathrm{G}}}{\partial f} & =\frac{q_{1} \sqrt{u}}{\left(1-q_{1} f\right)^{2}}, \text { and } \\
\frac{\partial u}{\partial f} & =2 \sigma_{\mathrm{Y}}^{2} q_{1}\left[\cosh \left(\frac{3}{2} q_{2} \frac{p^{\prime}}{\sigma_{\mathrm{Y}}}\right)-1\right] .
\end{aligned}
$$

Appendix F.3. GTN plastic normal

The plastic normal following Eq. (55) can be rewritten as

$$
\mathbf{N}_{\mathrm{G}}^{\mathrm{p}}=\frac{\partial \hat{\sigma}_{\mathrm{G}}}{\partial u} \frac{\partial u}{\partial \boldsymbol{\sigma}} .
$$

Its derivatives with respect to $\boldsymbol{\sigma}, \sigma_{\mathrm{Y}}$, and $\mathbf{Y}$ are given as follows

$$
\begin{aligned}
\frac{\partial \mathbf{N}_{\mathrm{G}}^{\mathrm{p}}}{\partial \boldsymbol{\sigma}} & =\frac{\partial^{2} \hat{\sigma}_{\mathrm{G}}}{\partial u^{2}} \frac{\partial u}{\partial \boldsymbol{\sigma}} \otimes \frac{\partial u}{\partial \boldsymbol{\sigma}}+\frac{\partial \hat{\sigma}_{\mathrm{G}}}{\partial u} \frac{\partial^{2} u}{\partial \boldsymbol{\sigma} \otimes \partial \boldsymbol{\sigma}}, \\
\frac{\partial \mathbf{N}_{\mathrm{G}}^{\mathrm{p}}}{\partial \sigma_{\mathrm{Y}}} & =\frac{\partial^{2} \hat{\sigma}_{\mathrm{G}}}{\partial u^{2}} \frac{\partial u}{\partial \sigma_{\mathrm{Y}}} \frac{\partial u}{\partial \boldsymbol{\sigma}}+\frac{\partial \hat{\sigma}_{\mathrm{G}}}{\partial u} \frac{\partial^{2} u}{\partial \boldsymbol{\sigma} \partial \sigma_{\mathrm{Y}}}, \text { and } \\
\frac{\partial \mathbf{N}_{\mathrm{G}}^{\mathrm{p}}}{\partial \mathbf{Y}} & =\left[\begin{array}{llll}
\frac{\partial \mathbf{N}_{\mathrm{G}}^{\mathrm{p}}}{\partial f} & 0 & 0 & 0
\end{array}\right] .
\end{aligned}
$$


Using Eqs. (F.11, F.15), Eqs. (F.16 - F.19) are completed by

$$
\begin{aligned}
\frac{\partial^{2} \hat{\sigma}_{\mathrm{G}}}{\partial u^{2}} & =-\frac{1}{4 u \sqrt{u}\left(1-q_{1} f\right)}, \\
\frac{\partial^{2} u}{\partial \boldsymbol{\sigma} \otimes \partial \boldsymbol{\sigma}} & =3 \mathcal{I}^{\text {dev }}+\frac{f q_{1} q_{2}^{2}}{2} \cosh \left(\frac{3}{2} q_{2} \frac{p^{\prime}}{\sigma_{\mathrm{Y}}}\right) \mathbf{I} \otimes \mathbf{I} \\
\frac{\partial^{2} u}{\partial \boldsymbol{\sigma} \partial \sigma_{\mathrm{Y}}} & =\left[f q_{1} q_{2} \sinh \left(\frac{3}{2} q_{2} \frac{p^{\prime}}{\sigma_{\mathrm{Y}}}\right)-\frac{3}{2} f q_{1} q_{2}^{2} \frac{p^{\prime}}{\sigma_{\mathrm{Y}}} \cosh \left(\frac{3}{2} q_{2} \frac{p^{\prime}}{\sigma_{\mathrm{Y}}}\right)\right] \mathbf{I}, \\
\frac{\partial^{2} \hat{\sigma}_{\mathrm{G}}}{\partial u \partial f} & =\frac{q_{1}}{2 \sqrt{u}\left(1-q_{1} f\right)^{2}}, \\
\frac{\partial^{2} u}{\partial \boldsymbol{\sigma} \partial f} & =\sigma_{\mathrm{Y}} q_{1} q_{2} \sinh \left(\frac{3}{2} q_{2} \frac{p^{\prime}}{\sigma_{\mathrm{Y}}}\right) \mathbf{I}, \text { and } \\
\frac{\partial \mathbf{N}_{\mathrm{G}}^{\mathrm{p}}}{\partial f} & =\left(\frac{\partial^{2} \hat{\sigma}_{\mathrm{G}}}{\partial u \partial f}+\frac{\partial^{2} \hat{\sigma}_{\mathrm{G}}}{\partial u^{2}} \frac{\partial u}{\partial f}\right) \frac{\partial u}{\partial \boldsymbol{\sigma}}+\frac{\partial \hat{\sigma}_{\mathrm{G}}}{\partial u} \frac{\partial^{2} u}{\partial \boldsymbol{\sigma} \partial f} .
\end{aligned}
$$

Appendix F.4. MPS-based Thomason yield function

The smoothed version of the Thomason effective stress $\hat{\sigma}_{\mathrm{T}}$ described in Eq. (E.3) is considered in the MPS-based Thomason yield function (75). For the sake of simplicity, Eq. (E.3) can be rewritten as

$$
\hat{\sigma}_{\mathrm{T}}=\frac{G}{C_{\mathrm{Tf}}}
$$

where

$$
\begin{gathered}
G(Z)=Z^{\frac{1}{n}}, Z\left(V_{1}, V_{2}\right)=V_{1}^{\frac{n}{2}}+V_{2}^{\frac{n}{2}}, V_{1}=\left(p^{\prime}+s\right)^{2}, V_{2}=\left(-p^{\prime}+s\right)^{2} \\
s=\frac{2}{3} \sigma_{\mathrm{eq}} F, F(\omega)=\cos \theta_{T}, \theta_{T}=\frac{1}{3} \arccos \left(1-a_{T}+a_{T} \omega\right)
\end{gathered}
$$

and $\omega$ is given by Eq. (57).

The derivatives of the MPS-based Thomason yield function following Eq. (75) with respect to $\boldsymbol{\sigma}, \sigma_{\mathrm{Y}}$, and $\mathbf{Y}$ are given as follows

$$
\begin{aligned}
\frac{\partial \Phi_{\mathrm{T}}}{\partial \boldsymbol{\sigma}} & =\frac{1}{\sigma_{\mathrm{Y}} C_{\mathrm{Tf}}}\left(\frac{\partial G}{\partial p^{\prime}} \frac{\partial p^{\prime}}{\partial \boldsymbol{\sigma}}+\frac{\partial G}{\partial s} \frac{\partial s}{\partial \sigma_{\mathrm{eq}}} \frac{\partial \sigma_{\mathrm{eq}}}{\partial \boldsymbol{\sigma}}+\frac{\partial G}{\partial s} \frac{\partial s}{\partial J_{3}} \frac{\partial J_{3}}{\partial \boldsymbol{\sigma}}\right), \\
\frac{\partial \Phi_{\mathrm{T}}}{\partial \sigma_{\mathrm{Y}}} & =-\frac{\hat{\sigma}_{\mathrm{T}}}{\sigma_{\mathrm{Y}}^{2}}, \text { and } \\
\frac{\partial \Phi_{\mathrm{T}}}{\partial \mathbf{Y}} & =-\frac{\hat{\sigma}_{\mathrm{T}}}{\sigma_{\mathrm{Y}} C_{\mathrm{Tf}}}\left[\begin{array}{llll}
0 & \frac{\partial C_{\mathrm{Tf}}}{\partial \chi} & \frac{\partial C_{\mathrm{Tf}}}{\partial W} & 0
\end{array}\right],
\end{aligned}
$$


where $\hat{\sigma}_{\mathrm{T}}$ is given by Eq. (F.26) and

$$
\begin{aligned}
\frac{\partial C_{\mathrm{Tf}}}{\partial \chi}= & -2 \chi\left[h\left(\frac{1-\chi}{W \chi}\right)^{2}+g \sqrt{\frac{1}{\chi}}\right] \\
& +\left(1-\chi^{2}\right)\left[-\frac{2 h}{W^{2}}\left(\frac{1}{\chi}-1\right) \frac{1}{\chi^{2}}-\frac{g}{2 \chi \sqrt{\chi}}\right], \text { and } \\
\frac{\partial C_{\mathrm{Tf}}}{\partial W}= & \left(1-\chi^{2}\right)\left[-\frac{2 h}{W}\left(\frac{1-\chi}{W \chi}\right)^{2}\right] .
\end{aligned}
$$

Using Eqs. (F.2 - F.6, F.27), Eq. (F.28) is completed by

$$
\begin{aligned}
\frac{\partial s}{\partial \sigma_{\mathrm{eq}}} & =\frac{2}{3}\left(F+\sigma_{\mathrm{eq}} F^{\prime} \frac{\partial \omega}{\partial \sigma_{\mathrm{eq}}}\right), \frac{\partial s}{\partial J_{3}}=\frac{2}{3} \sigma_{\mathrm{eq}} F^{\prime} \frac{\partial \omega}{\partial J_{3}}, \text { and } \\
\frac{\partial G}{\partial U_{i}} & =G^{\prime} \sum_{k=1}^{2} \frac{\partial Z}{\partial V_{k}} \frac{\partial V_{k}}{\partial U_{i}} \text { for } U_{i}=p^{\prime} \text { or } s,
\end{aligned}
$$

where

$$
\begin{aligned}
& F^{\prime}=-\left(\sin \theta_{T}\right) \theta_{T}^{\prime}, \theta_{T}^{\prime}=\frac{-a_{T}}{3 \sqrt{1-\left(1-a_{T}+a_{T} \omega\right)^{2}}}, \\
& \frac{\partial \omega}{\partial \sigma_{\mathrm{eq}}}=-\frac{81}{2} \frac{J_{3}}{\sigma_{\mathrm{eq}}{ }^{2}}, \frac{\partial \omega}{\partial J_{3}}=\frac{27}{2} \frac{1}{\sigma_{\mathrm{eq}}^{3}}, \\
& G^{\prime}=\frac{1}{n} Z^{\frac{1}{n}-1}, \frac{\partial Z}{\partial V_{i}}=\frac{n}{2} V_{i}^{\frac{n}{2}-1}, \frac{\partial V_{1}}{\partial p^{\prime}}=2\left(p^{\prime}+s\right), \frac{\partial V_{1}}{\partial s}=2\left(p^{\prime}+s\right), \\
& \frac{\partial V_{2}}{\partial p^{\prime}}=2\left(p^{\prime}-s\right), \text { and } \frac{\partial V_{2}}{\partial s}=2\left(-p^{\prime}+s\right) .
\end{aligned}
$$

Appendix F.5. MPS-based Thomason plastic normal

The MPS-based Thomason plastic normal $\mathbf{N}_{\mathrm{T}}^{\mathrm{p}}$ following Eq. (77) reads

$$
\mathbf{N}_{\mathrm{T}}^{\mathrm{p}}=\sigma_{\mathrm{Y}} \frac{\partial \Phi_{\mathrm{T}}}{\partial \boldsymbol{\sigma}}=\frac{\partial \hat{\sigma}_{\mathrm{T}}}{\partial \boldsymbol{\sigma}}=\frac{1}{C_{\mathrm{Tf}}}\left(\frac{\partial G}{\partial p^{\prime}} \frac{\partial p^{\prime}}{\partial \boldsymbol{\sigma}}+\frac{\partial G}{\partial s} \frac{\partial s}{\partial \sigma_{\mathrm{eq}}} \frac{\partial \sigma_{\mathrm{eq}}}{\partial \boldsymbol{\sigma}}+\frac{\partial G}{\partial s} \frac{\partial s}{\partial J_{3}} \frac{\partial J_{3}}{\partial \boldsymbol{\sigma}}\right)
$$


where $\frac{\partial \Phi_{\mathrm{T}}}{\partial \boldsymbol{\sigma}}$ is given by Eq. (F.28). The derivatives of $\mathbf{N}_{\mathrm{T}}^{\mathrm{p}}$ with respect to $\boldsymbol{\sigma}, \sigma_{\mathrm{Y}}$, and $\mathbf{Y}$ are given as follows

$$
\begin{aligned}
& \frac{\partial \mathbf{N}_{\mathrm{T}}^{\mathrm{p}}}{\partial \boldsymbol{\sigma}}=\frac{1}{C_{\mathrm{Tf}}}\left[\frac{\partial^{2} G}{\partial{p^{\prime}}^{2}} \frac{\partial p^{\prime}}{\partial \boldsymbol{\sigma}}+\frac{\partial^{2} G}{\partial \sigma_{\mathrm{eq}} \partial p^{\prime}} \frac{\partial \sigma_{\mathrm{eq}}}{\partial \boldsymbol{\sigma}}+\frac{\partial^{2} G}{\partial J_{3} \partial p^{\prime}} \frac{\partial J_{3}}{\partial \boldsymbol{\sigma}}\right] \otimes \frac{\partial p^{\prime}}{\partial \boldsymbol{\sigma}} \\
& +\frac{1}{C_{\mathrm{Tf}}}\left[\frac{\partial^{2} G}{\partial p^{\prime} \partial \sigma_{\mathrm{eq}}} \frac{\partial p^{\prime}}{\partial \boldsymbol{\sigma}}+\frac{\partial^{2} G}{\partial \sigma_{\mathrm{eq}}{ }^{2}} \frac{\partial \sigma_{\mathrm{eq}}}{\partial \boldsymbol{\sigma}}+\frac{\partial^{2} G}{\partial J_{3} \partial \sigma_{\mathrm{eq}}} \frac{\partial J_{3}}{\partial \boldsymbol{\sigma}}\right] \otimes \frac{\partial \sigma_{\mathrm{eq}}}{\partial \boldsymbol{\sigma}} \\
& +\frac{1}{C_{\mathrm{Tf}}}\left[\frac{\partial^{2} G}{\partial p^{\prime} \partial J_{3}} \frac{\partial p^{\prime}}{\partial \boldsymbol{\sigma}}+\frac{\partial^{2} G}{\partial \sigma_{\mathrm{eq}} \partial J_{3}} \frac{\partial \sigma_{\mathrm{eq}}}{\partial \boldsymbol{\sigma}}+\frac{\partial^{2} G}{\partial J_{3}^{2}} \frac{\partial J_{3}}{\partial \boldsymbol{\sigma}}\right] \otimes \frac{\partial J_{3}}{\partial \boldsymbol{\sigma}} \\
& +\frac{1}{C_{\mathrm{Tf}}} \frac{\partial G}{\partial \sigma_{\mathrm{eq}}} \frac{\partial^{2} \sigma_{\mathrm{eq}}}{\partial \boldsymbol{\sigma} \otimes \partial \boldsymbol{\sigma}}+\frac{1}{C_{\mathrm{Tf}}} \frac{\partial G}{\partial J_{3}} \frac{\partial^{2} J_{3}}{\partial \boldsymbol{\sigma} \otimes \partial \boldsymbol{\sigma}}, \\
& \frac{\partial \mathbf{N}_{\mathrm{T}}^{\mathrm{p}}}{\partial \sigma_{\mathrm{Y}}}=\mathbf{0}, \text { and } \\
& \frac{\partial \mathbf{N}_{\mathrm{T}}^{\mathrm{p}}}{\partial \mathbf{Y}}=-\frac{\mathbf{N}_{\mathrm{T}}^{\mathrm{p}}}{C_{\mathrm{Tf}}}\left[\begin{array}{llll}
0 & \frac{\partial C_{\mathrm{Tf}}}{\partial \chi} & \frac{\partial C_{\mathrm{Tf}}}{\partial W} & 0
\end{array}\right],
\end{aligned}
$$

where

$$
\begin{aligned}
\frac{\partial^{2} G}{\partial p^{\prime} \partial \sigma_{\mathrm{eq}}}=\frac{\partial^{2} G}{\partial \sigma_{\mathrm{eq}} \partial p^{\prime}} & =\frac{\partial^{2} G}{\partial p^{\prime} \partial s} \frac{\partial s}{\partial \sigma_{\mathrm{eq}}} \\
\frac{\partial^{2} G}{\partial p^{\prime} \partial J_{3}}=\frac{\partial^{2} G}{\partial J_{3} \partial p^{\prime}} & =\frac{\partial^{2} G}{\partial p^{\prime} \partial s} \frac{\partial s}{\partial J_{3}} \\
\frac{\partial^{2} G}{\partial \sigma_{\mathrm{eq}} \partial J_{3}}=\frac{\partial^{2} G}{\partial J_{3} \partial \sigma_{\mathrm{eq}}} & =\frac{\partial^{2} G}{\partial s^{2}} \frac{\partial s}{\partial \sigma_{\mathrm{eq}}} \frac{\partial s}{\partial J_{3}}+\frac{\partial G}{\partial s} \frac{\partial^{2} s}{\partial \sigma_{\mathrm{eq}} \partial J_{3}}, \\
\frac{\partial^{2} G}{\partial \sigma_{\mathrm{eq}}^{2}} & =\frac{\partial^{2} G}{\partial s^{2}} \frac{\partial s}{\partial \sigma_{\mathrm{eq}}} \frac{\partial s}{\partial \sigma_{\mathrm{eq}}}+\frac{\partial G}{\partial s} \frac{\partial^{2} s}{\partial \sigma_{\mathrm{eq}}^{2}}, \\
\frac{\partial^{2} G}{\partial J_{3}^{2}} & =\frac{\partial^{2} G}{\partial s^{2}} \frac{\partial s}{\partial J_{3}} \frac{\partial s}{\partial J_{3}}+\frac{\partial G}{\partial s} \frac{\partial^{2} s}{\partial J_{3}^{2}}
\end{aligned}
$$


Using Eqs. (F.2 - F.6, F.34), Eqs. (F.39 - F.43) are completed by

$$
\begin{aligned}
\frac{\partial^{2} s}{\partial \sigma_{\mathrm{eq}}{ }^{2}}= & \frac{2}{3}\left(2 F^{\prime} \frac{\partial \omega}{\partial \sigma_{\mathrm{eq}}}+\sigma_{\mathrm{eq}} F^{\prime \prime} \frac{\partial \omega}{\partial \sigma_{\mathrm{eq}}} \frac{\partial \omega}{\partial \sigma_{\mathrm{eq}}}+\sigma_{\mathrm{eq}} F^{\prime} \frac{\partial^{2} \omega}{\partial \sigma_{\mathrm{eq}}{ }^{2}}\right) \\
\frac{\partial^{2} s}{\partial \sigma_{\mathrm{eq}} \partial J_{3}}=\frac{\partial^{2} s}{\partial J_{3} \partial \sigma_{\mathrm{eq}}}= & \frac{2}{3}\left(F^{\prime} \frac{\partial \omega}{\partial J_{3}}+\sigma_{\mathrm{eq}} F^{\prime \prime} \frac{\partial \omega}{\partial \sigma_{\mathrm{eq}}} \frac{\partial \omega}{\partial J_{3}}+\sigma_{\mathrm{eq}} F^{\prime} \frac{\partial^{2} \omega}{\partial \sigma_{\mathrm{eq}} \partial J_{3}}\right) \\
\frac{\partial^{2} s}{\partial J_{3}^{2}}= & \frac{2}{3} \sigma_{\mathrm{eq}}\left(F^{\prime \prime} \frac{\partial \omega}{\partial J_{3}} \frac{\partial \omega}{\partial J_{3}}+F^{\prime} \frac{\partial^{2} \omega}{\partial J_{3}^{2}}\right), \text { and } \\
\frac{\partial^{2} G}{\partial U_{i} \partial U_{j}}= & G^{\prime \prime} \sum_{k=1}^{2} \frac{\partial Z}{\partial V_{k}} \frac{\partial V_{k}}{\partial U_{i}} \sum_{m=1}^{2} \frac{\partial Z}{\partial V_{m}} \frac{\partial V_{m}}{\partial U_{j}} \\
& +G^{\prime} \sum_{k=1}^{2} \frac{\partial^{2} Z}{\partial V_{k}^{2}} \frac{\partial V_{k}}{\partial U_{i}} \frac{\partial V_{k}}{\partial U_{j}}+G^{\prime} \sum_{k=1}^{2} \frac{\partial Z}{\partial V_{k}} \frac{\partial^{2} V_{k}}{\partial U_{i} \partial U_{j}} \text { for } U_{i}=p^{\prime} \text { or } s,
\end{aligned}
$$

where

$$
\begin{aligned}
& F^{\prime \prime}=-\left(\cos \theta_{T}\right) \theta_{T}^{\prime 2}-\left(\sin \theta_{T}\right) \theta_{T}^{\prime \prime}, \\
& \theta_{T}^{\prime \prime}=\theta_{T}^{\prime} \frac{a_{T}\left(1-a_{T}+a_{T} \omega\right)}{1-\left(1-a_{T}+a_{T} \omega\right)^{2}}, \\
& \frac{\partial^{2} \omega}{\partial \sigma_{\mathrm{eq}}^{2}}=\frac{324 J_{3}}{2 \sigma_{\mathrm{eq}}{ }^{5}}, \frac{\partial^{2} \omega}{\partial \sigma_{\mathrm{eq}} \partial J_{3}}=\frac{\partial^{2} \omega}{\partial J_{3} \partial \sigma_{\mathrm{eq}}}=-\frac{81}{2 \sigma_{\mathrm{eq}}{ }^{4}}, \frac{\partial^{2} \omega}{\partial J_{3}^{2}}=0, \\
& G^{\prime \prime}=\frac{1}{n}\left(\frac{1}{n}-1\right) Z^{\frac{1}{n}-2}, \frac{\partial^{2} Z}{\partial V_{k}^{2}}=\frac{n}{2}\left(\frac{n}{2}-1\right) V_{k}^{\frac{n}{2}-2}, \\
& \frac{\partial V_{1}^{2}}{\partial p^{\prime} \partial p^{\prime}}=\frac{\partial V_{1}^{2}}{\partial p^{\prime} \partial s}=\frac{\partial V_{1}^{2}}{\partial s \partial p^{\prime}}=\frac{\partial V_{1}^{2}}{\partial s \partial s}=2, \text { and } \\
& \frac{\partial V_{2}^{2}}{\partial p^{\prime} \partial p^{\prime}}=-\frac{\partial V_{2}^{2}}{\partial p^{\prime} \partial s}=-\frac{\partial V_{2}^{2}}{\partial s \partial p^{\prime}}=\frac{\partial V_{2}^{2}}{\partial s \partial s}=2 .
\end{aligned}
$$

Appendix F.6. MSS-based yield function

The smoothed version of the MSS-based yield function (84) is obtained using the shear effective stress following Eq. (E.5). For the sake of simplicity, Eq. (E.5) can be rewritten as

$$
\hat{\sigma}_{\mathrm{S}}=\frac{\sigma_{\mathrm{eq}}}{C_{\mathrm{Sf}}} P(\omega),
$$

where

$$
P(\omega)=\frac{\sin \theta_{S}}{2}+\frac{\sqrt{3} \cos \theta_{S}}{2}, \theta_{S}=\frac{1}{3} \arccos \left(a_{S} \omega\right)
$$


and $\omega$ is given by Eq. (57). The derivatives of the MSS-based yield function (84) with respect to $\boldsymbol{\sigma}, \sigma_{\mathrm{Y}}$, and $\mathbf{Y}$, are given as follows

$$
\begin{aligned}
& \frac{\partial \Phi_{\mathrm{S}}}{\partial \boldsymbol{\sigma}}=\frac{1}{\sigma_{\mathrm{Y}}}\left(\frac{\partial \hat{\sigma}_{\mathrm{S}}}{\partial \sigma_{\mathrm{eq}}} \frac{\partial \sigma_{\mathrm{eq}}}{\partial \boldsymbol{\sigma}}+\frac{\partial \hat{\sigma}_{\mathrm{S}}}{\partial J_{3}} \frac{\partial J_{3}}{\partial \boldsymbol{\sigma}}\right), \\
& \frac{\partial \Phi_{\mathrm{S}}}{\partial \sigma_{\mathrm{Y}}}=-\frac{\hat{\sigma}_{\mathrm{S}}}{\sigma_{\mathrm{Y}}^{2}}, \text { and } \\
& \frac{\partial \Phi_{\mathrm{S}}}{\partial \mathbf{Y}}=\frac{\hat{\sigma}_{\mathrm{S}}}{\sigma_{\mathrm{Y}} C_{\mathrm{Sf}}}\left[\begin{array}{llll}
0 & 2 \xi \chi & 0 & 0
\end{array}\right],
\end{aligned}
$$

where

$$
\begin{aligned}
& \frac{\partial \hat{\sigma}_{\mathrm{S}}}{\partial \sigma_{\mathrm{eq}}}=\frac{1}{C_{\mathrm{Sf}}}\left(P+\sigma_{\mathrm{eq}} P^{\prime} \frac{\partial \omega}{\partial \sigma_{\mathrm{eq}}}\right), \frac{\partial \hat{\sigma}_{\mathrm{S}}}{\partial J_{3}}=\frac{\sigma_{\mathrm{eq}}}{C_{\mathrm{Sf}}} P^{\prime} \frac{\partial \omega}{\partial J_{3}}, \\
& P^{\prime}=\left(\frac{\cos \theta_{S}}{2}-\frac{\sqrt{3} \sin \theta_{S}}{2}\right) \theta_{S}^{\prime}, \text { and } \theta_{S}^{\prime}=\frac{-a_{S}}{3 \sqrt{1-\left(a_{S} \omega\right)^{2}}},
\end{aligned}
$$

in combination with Eqs. (F.2 - F.6, F.34, F.49, F.50).

Appendix F.7. MSS-based yield plastic normal

The MSS-based plastic normal $\mathbf{N}_{\mathrm{S}}^{\mathrm{p}}$ following Eq. (86) reads

$$
\mathbf{N}_{\mathrm{S}}^{\mathrm{p}}=\sigma_{\mathrm{Y}} \frac{\partial \Phi_{\mathrm{S}}}{\partial \boldsymbol{\sigma}}=\frac{\partial \hat{\sigma}_{\mathrm{S}}}{\partial \boldsymbol{\sigma}}=\frac{\partial \hat{\sigma}_{\mathrm{S}}}{\partial \sigma_{\mathrm{eq}}} \frac{\partial \sigma_{\mathrm{eq}}}{\partial \boldsymbol{\sigma}}+\frac{\partial \hat{\sigma}_{\mathrm{S}}}{\partial J_{3}} \frac{\partial J_{3}}{\partial \boldsymbol{\sigma}},
$$

where $\frac{\partial \Phi_{\mathrm{S}}}{\partial \boldsymbol{\sigma}}$ is given in Eq. (F.51). The derivatives of $\mathbf{N}_{\mathrm{S}}^{\mathrm{p}}$ with respect to $\boldsymbol{\sigma}, \sigma_{\mathrm{Y}}$, and $\mathbf{Y}$ are given as follows

$$
\begin{aligned}
& \frac{\partial \mathbf{N}_{\mathrm{S}}^{\mathrm{p}}}{\partial \boldsymbol{\sigma}}=\left[\frac{\partial^{2} \hat{\sigma}_{\mathrm{S}}}{\partial \sigma_{\mathrm{eq}}{ }^{2}} \frac{\partial \sigma_{\mathrm{eq}}}{\partial \boldsymbol{\sigma}}+\frac{\partial^{2} \hat{\sigma}_{\mathrm{S}}}{\partial J_{3} \partial \sigma_{\mathrm{eq}}} \frac{\partial J_{3}}{\partial \boldsymbol{\sigma}}\right] \otimes \frac{\partial \sigma_{\mathrm{eq}}}{\partial \boldsymbol{\sigma}} \\
& +\left[\frac{\partial^{2} \hat{\sigma}_{\mathrm{S}}}{\partial \sigma_{\mathrm{eq}} \partial J_{3}} \frac{\partial \sigma_{\mathrm{eq}}}{\partial \boldsymbol{\sigma}}+\frac{\partial^{2} \hat{\sigma}_{\mathrm{S}}}{\partial J_{3}^{2}} \frac{\partial J_{3}}{\partial \boldsymbol{\sigma}}\right] \otimes \frac{\partial J_{3}}{\partial \boldsymbol{\sigma}} \\
& +\frac{\partial \hat{\sigma}_{\mathrm{S}}}{\partial \sigma_{\mathrm{eq}}} \frac{\partial^{2} \sigma_{\mathrm{eq}}}{\partial \boldsymbol{\sigma} \otimes \partial \boldsymbol{\sigma}}+\frac{\partial \hat{\sigma}_{\mathrm{S}}}{\partial J_{3}} \frac{\partial^{2} J_{3}}{\partial \boldsymbol{\sigma} \otimes \partial \boldsymbol{\sigma}}, \\
& \frac{\partial \mathbf{N}_{\mathrm{S}}^{\mathrm{p}}}{\partial \sigma_{\mathrm{Y}}}=\mathbf{0} \text {, and } \\
& \frac{\partial \mathbf{N}_{\mathrm{S}}^{\mathrm{p}}}{\partial \mathbf{Y}}=\frac{\mathbf{N}_{\mathrm{S}}^{\mathrm{p}}}{C_{\mathrm{Sf}}}\left[\begin{array}{llll}
0 & 2 \xi \chi & 0 & 0
\end{array}\right],
\end{aligned}
$$


where

$$
\begin{aligned}
\frac{\partial^{2} \hat{\sigma}_{\mathrm{S}}}{\partial \sigma_{\mathrm{eq}}{ }^{2}} & =\frac{1}{C_{\mathrm{Sf}}}\left[2 P^{\prime} \frac{\partial \omega}{\partial \sigma_{\mathrm{eq}}}+\sigma_{\mathrm{eq}} P^{\prime \prime}\left(\frac{\partial \omega}{\partial \sigma_{\mathrm{eq}}}\right)^{2}+\sigma_{\mathrm{eq}} P^{\prime} \frac{\partial^{2} \omega}{\partial \sigma_{\mathrm{eq}} \partial \sigma_{\mathrm{eq}}}\right], \\
\frac{\partial^{2} \hat{\sigma}_{\mathrm{S}}}{\partial \sigma_{\mathrm{eq}} \partial J_{3}}=\frac{\partial^{2} \hat{\sigma}_{\mathrm{S}}}{\partial J_{3} \partial \sigma_{\mathrm{eq}}} & =\frac{1}{C_{\mathrm{Sf}}}\left(P^{\prime} \frac{\partial \omega}{\partial J_{3}}+\sigma_{\mathrm{eq}} P^{\prime \prime} \frac{\partial \omega}{\partial J_{3}} \frac{\partial \omega}{\partial \sigma_{\mathrm{eq}}}+\sigma_{\mathrm{eq}} P^{\prime} \frac{\partial^{2} \omega}{\partial \sigma_{\mathrm{eq}} \partial J_{3}}\right) \\
\frac{\partial^{2} \hat{\sigma}_{\mathrm{S}}}{\partial J_{3}^{2}} & =\frac{\sigma_{\mathrm{eq}}}{C_{\mathrm{Sf}}}\left[P^{\prime \prime}\left(\frac{\partial \omega}{\partial J_{3}}\right)^{2}+P^{\prime} \frac{\partial^{2} \omega}{\partial J_{3} \partial J_{3}}\right] \\
P^{\prime \prime} & =\left(\frac{-\sin \theta_{S}}{2}-\frac{\sqrt{3} \cos \theta_{S}}{2}\right) \theta_{S}^{\prime 2}+ \\
\theta_{S}^{\prime \prime} & =\theta_{S}^{\prime} \frac{a_{S}^{2} \omega}{1-\left(a_{S} \omega\right)^{2}},
\end{aligned}
$$

in combination with Eqs. (F.2 - F.6, F.34, F.48, F.50, F.54).

\section{Appendix G. Convexity of the maximum principal stress and of the maximum shear stress in the stress space}

Let $\mathbf{n}_{1}, \mathbf{n}_{2}, \mathbf{n}_{3}$ be the eigenvectors respectively associated to the three ordered eigenvalues given in Eq. (71) of the stress tensor $\boldsymbol{\sigma}$. One has the orthogonal properties

$$
\mathbf{n}_{i} \cdot \mathbf{n}_{j}=\left\{\begin{array}{l}
1 \text { if } i=j \\
0 \text { if } i \neq j
\end{array} .\right.
$$

The eigenvalue $\sigma_{1}$ relates to its eigenvector $\mathbf{n}_{1}$ by the equality

$$
\boldsymbol{\sigma} \cdot \mathbf{n}_{1}=\sigma_{1} \mathbf{n}_{1}
$$

Applying the time derivative, the last equation yields

$$
\dot{\boldsymbol{\sigma}} \cdot \mathbf{n}_{1}+\boldsymbol{\sigma} \cdot \dot{\mathbf{n}}_{1}=\dot{\sigma}_{1} \mathbf{n}_{1}+\sigma_{1} \dot{\mathbf{n}}_{1}
$$

Performimg the inner product of Eq. (G.3) with $\mathbf{n}_{1}$ and using $\mathbf{n}_{1} \cdot \boldsymbol{\sigma}=\boldsymbol{\sigma} \cdot \mathbf{n}_{1}=\sigma_{1} \mathbf{n}_{1}$ (because $\boldsymbol{\sigma}$ is symmetric) yield

$$
\mathbf{n}_{1} \cdot \dot{\boldsymbol{\sigma}} \cdot \mathbf{n}_{1}=\dot{\sigma}_{1}
$$


leading to

$$
\frac{\partial \sigma_{1}}{\partial \boldsymbol{\sigma}}=\mathbf{n}_{1} \otimes \mathbf{n}_{1}
$$

Performing the inner product of Eq. (G.3) with $\mathbf{n}_{2}$ and $\mathbf{n}_{3}$ and using the orthonormal properties between eigenvectors, one successively has

$$
\left\{\begin{array}{l}
\mathbf{n}_{2} \cdot \dot{\boldsymbol{\sigma}} \cdot \mathbf{n}_{1}+\mathbf{n}_{2} \cdot \boldsymbol{\sigma} \cdot \dot{\mathbf{n}}_{1}=\sigma_{1} \dot{\mathbf{n}}_{1} \cdot \mathbf{n}_{2}, \text { and } \\
\mathbf{n}_{3} \cdot \dot{\boldsymbol{\sigma}} \cdot \mathbf{n}_{1}+\mathbf{n}_{3} \cdot \boldsymbol{\sigma} \cdot \dot{\mathbf{n}}_{1}=\sigma_{1} \dot{\mathbf{n}}_{1} \cdot \mathbf{n}_{3}
\end{array}\right.
$$

Using $\mathbf{n}_{i} \cdot \boldsymbol{\sigma}=\boldsymbol{\sigma} \cdot \mathbf{n}_{i}=\sigma_{i} \mathbf{n}_{i}$ for $i=2,3$, Eqs. (G.6) can be rewritten as

$$
\left\{\begin{array}{l}
\mathbf{n}_{2} \cdot \dot{\boldsymbol{\sigma}} \cdot \mathbf{n}_{1}=\left(\sigma_{1}-\sigma_{2}\right) \dot{\mathbf{n}}_{1} \cdot \mathbf{n}_{2}, \text { and } \\
\mathbf{n}_{3} \cdot \dot{\boldsymbol{\sigma}} \cdot \mathbf{n}_{1}=\left(\sigma_{1}-\sigma_{3}\right) \dot{\mathbf{n}}_{1} \cdot \mathbf{n}_{3} .
\end{array}\right.
$$

Since the three eigenvectors form an orthonormal basis with $\mathbf{n}_{1} \cdot \mathbf{n}_{1}=1$ and $\dot{\mathbf{n}}_{1} \cdot \mathbf{n}_{1}=0$, one has

$$
\dot{\mathbf{n}}_{1}=\dot{\mathbf{n}}_{1} \cdot \mathbf{n}_{1} \mathbf{n}_{1}+\dot{\mathbf{n}}_{1} \cdot \mathbf{n}_{2} \mathbf{n}_{2}+\dot{\mathbf{n}}_{1} \cdot \mathbf{n}_{3} \mathbf{n}_{3}=\dot{\mathbf{n}}_{1} \cdot \mathbf{n}_{2} \mathbf{n}_{2}+\dot{\mathbf{n}}_{1} \cdot \mathbf{n}_{3} \mathbf{n}_{3},
$$

or, using Eq. (G.7),

$$
\dot{\mathbf{n}}_{1}=\left[\frac{\mathbf{n}_{2} \otimes \mathbf{n}_{2} \otimes \mathbf{n}_{1}}{\sigma_{1}-\sigma_{2}}+\frac{\mathbf{n}_{3} \otimes \mathbf{n}_{3} \otimes \mathbf{n}_{1}}{\sigma_{1}-\sigma_{3}}\right]: \dot{\boldsymbol{\sigma}} .
$$

Combining Eqs. (G.5, G.9), one has

$$
\begin{aligned}
\frac{\partial^{2} \sigma_{1}}{\partial \boldsymbol{\sigma} \otimes \partial \boldsymbol{\sigma}}=2 \quad & {\left[\frac{\operatorname{sym}\left(\mathbf{n}_{1} \otimes \mathbf{n}_{2}\right) \otimes \operatorname{sym}\left(\mathbf{n}_{1} \otimes \mathbf{n}_{2}\right)}{\sigma_{1}-\sigma_{2}}\right.} \\
& \left.+\frac{\operatorname{sym}\left(\mathbf{n}_{1} \otimes \mathbf{n}_{3}\right) \otimes \operatorname{sym}\left(\mathbf{n}_{1} \otimes \mathbf{n}_{3}\right)}{\sigma_{1}-\sigma_{3}}\right],
\end{aligned}
$$

where $\operatorname{sym}(\bullet)=0.5(\bullet+\bullet T)$ for $\bullet$ being an arbitrary second order tensor. Considering an arbitrary symmetrical tensor $\boldsymbol{\beta}$, one always has

$$
\boldsymbol{\beta}: \frac{\partial^{2} \sigma_{1}}{\partial \boldsymbol{\sigma} \otimes \partial \boldsymbol{\sigma}}: \boldsymbol{\beta}=2\left[\frac{\left(\mathbf{n}_{1} \cdot \boldsymbol{\beta} \cdot \mathbf{n}_{2}\right)^{2}}{\sigma_{1}-\sigma_{2}}+\frac{\left(\mathbf{n}_{1} \cdot \boldsymbol{\beta} \cdot \mathbf{n}_{3}\right)^{2}}{\sigma_{1}-\sigma_{3}}\right] \geq 0,
$$

which implies $\frac{\partial \sigma_{1}}{\partial \boldsymbol{\sigma} \otimes \partial \boldsymbol{\sigma}}$ is positive semi-definite. As a result, $\sigma_{1}$ is a convex function.

The maximum shear stress is given by

$$
\tau_{\max }=\frac{\sigma_{1}-\sigma_{3}}{2} .
$$


Using the same procedure as to obtain Eq. (G.10), one has

$$
\begin{aligned}
\frac{\partial \sigma_{3}}{\partial \boldsymbol{\sigma} \otimes \partial \boldsymbol{\sigma}}=-2 & {\left[\frac{\operatorname{sym}\left(\mathbf{n}_{3} \otimes \mathbf{n}_{2}\right) \otimes \operatorname{sym}\left(\mathbf{n}_{3} \otimes \mathbf{n}_{2}\right)}{\sigma_{2}-\sigma_{3}}\right.} \\
& \left.+\frac{\operatorname{sym}\left(\mathbf{n}_{3} \otimes \mathbf{n}_{1}\right) \otimes \operatorname{sym}\left(\mathbf{n}_{3} \otimes \mathbf{n}_{1}\right)}{\sigma_{1}-\sigma_{3}}\right],
\end{aligned}
$$

which is a negative semi-definite fourth order tensor. The Hessian of $\tau_{\max }$, i.e.

$$
\frac{\partial^{2} \tau_{\max }}{\partial \boldsymbol{\sigma} \otimes \partial \boldsymbol{\sigma}}=\frac{1}{2}\left(\frac{\partial^{2} \sigma_{1}}{\partial \boldsymbol{\sigma} \otimes \partial \boldsymbol{\sigma}}-\frac{\partial^{2} \sigma_{3}}{\partial \boldsymbol{\sigma} \otimes \partial \boldsymbol{\sigma}}\right)
$$

is thus positive semi-definite. As a result, $\tau_{\max }$ is a convex function.

\section{Appendix H. Void evolution}

Appendix H.1. Void evolution during growth phase

Evolution laws (66) must be integrated in the time interval $\left[t_{n}, t_{n+1}\right]$. With respect to the values at the previous time step $\mathbf{Y}_{n}=\left[\begin{array}{llll}f_{n} & \chi_{n} & W_{n} & \lambda_{n}\end{array}\right]^{T}$, the values $\mathbf{Y}=\left[\begin{array}{llll}f & \chi & W & \lambda\end{array}\right]^{T}$ at time $t$ are estimated as follows:

- The first equation of the system (66) can be approximated in the explicit form

$$
\Delta f=\left(1-f_{n}-\Delta f\right) \Delta \bar{\varepsilon}_{\mathrm{v}}+A_{n} \Delta \bar{\varepsilon}_{\mathrm{m}}+k_{\omega} \phi_{\eta} \phi_{\omega}\left(f_{n}+\Delta f\right) \Delta \bar{\varepsilon}_{\mathrm{d}},
$$

where $\Delta f=f-f_{n}$. The last equation leads to

$$
f=f_{n}+\Delta f=f_{n}+\frac{u}{v},
$$

where

$$
\begin{aligned}
& u=\left(1-f_{n}\right) \Delta \bar{\varepsilon}_{\mathrm{v}}+A_{n} \Delta \bar{\varepsilon}_{\mathrm{m}}+k_{\omega} \phi_{\eta} \phi_{\omega} f_{n} \Delta \bar{\varepsilon}_{\mathrm{d}}, \text { and } \\
& v=1+\Delta \bar{\varepsilon}_{\mathrm{v}}-k_{\omega} \phi_{\eta} \phi_{\omega} \Delta \bar{\varepsilon}_{\mathrm{d}} .
\end{aligned}
$$

- The value of $W$ remains constant: $W=W_{n}=W_{0}$.

- The value of $\lambda$ is given by

$$
\lambda=\lambda_{n} \exp \left(\kappa \Delta \bar{\varepsilon}_{\mathrm{d}}\right)
$$

- The value of $\chi$ follows Eq. (62) as

$$
\chi=\left(\frac{3 f \lambda}{2 W_{0}}\right)^{\frac{1}{3}}=\chi_{n}\left(\frac{f \lambda}{f_{n} \lambda_{n}}\right)^{\frac{1}{3}} .
$$


Finally, the derivatives of the porosity $f$ are first estimated as follows:

$$
\begin{aligned}
\frac{\partial f}{\partial \boldsymbol{\sigma}}= & \left(\frac{1}{v} \frac{\partial u}{\partial \eta}-\frac{\Delta f}{v} \frac{\partial v}{\partial \eta}\right)\left(\frac{\partial \eta}{\partial p^{\prime}} \frac{\partial p^{\prime}}{\partial \boldsymbol{\sigma}}+\frac{\partial \eta}{\partial \sigma_{\mathrm{eq}}} \frac{\partial \sigma_{\mathrm{eq}}}{\partial \boldsymbol{\sigma}}\right) \\
& +\left(\frac{1}{v} \frac{\partial u}{\partial \omega}-\frac{\Delta f}{v} \frac{\partial v}{\partial \omega}\right)\left(\frac{\partial \omega}{\partial \sigma_{\mathrm{eq}}} \frac{\partial \sigma_{\mathrm{eq}}}{\partial \boldsymbol{\sigma}}+\frac{\partial \omega}{\partial J_{3}} \frac{\partial J_{3}}{\partial \boldsymbol{\sigma}}\right), \\
\frac{\partial f}{\partial \bar{\varepsilon}_{\mathrm{v}}}= & \frac{1}{v} \frac{\partial u}{\partial \bar{\varepsilon}_{\mathrm{v}}}-\frac{\Delta f}{v} \frac{\partial v}{\partial \bar{\varepsilon}_{\mathrm{v}}} \\
\frac{\partial f}{\partial \bar{\varepsilon}_{\mathrm{m}}}= & \frac{1}{v} \frac{\partial u}{\partial \bar{\varepsilon}_{\mathrm{m}}}, \text { and } \\
\frac{\partial f}{\partial \bar{\varepsilon}_{\mathrm{d}}}= & \frac{1}{v} \frac{\partial u}{\partial \bar{\varepsilon}_{\mathrm{d}}}-\frac{\Delta f}{v} \frac{\partial v}{\partial \bar{\varepsilon}_{\mathrm{d}}},
\end{aligned}
$$

where

$$
\begin{aligned}
& \frac{\partial u}{\partial \eta}=k_{\omega} f_{n} \phi_{\eta}^{\prime} \phi_{\omega} \Delta \bar{\varepsilon}_{\mathrm{d}}, \frac{\partial v}{\partial \eta}=-k_{\omega} \phi_{\eta}^{\prime} \phi_{\omega} \Delta \bar{\varepsilon}_{\mathrm{d}}, \\
& \frac{\partial u}{\partial \omega}=k_{\omega} f_{n} \phi_{\eta} \phi_{\omega}^{\prime} \Delta \bar{\varepsilon}_{\mathrm{d}}, \frac{\partial v}{\partial \omega}=-k_{\omega} \phi_{\eta} \phi_{\omega}^{\prime} \Delta \bar{\varepsilon}_{\mathrm{d}}, \\
& \phi_{\eta}^{\prime}=-\phi_{\eta} \frac{\eta}{\eta_{s}^{2}}, \phi_{\omega}^{\prime}=-2 \omega, \frac{\partial \omega}{\partial \sigma_{\mathrm{eq}}}=-\frac{3 \omega}{\sigma_{\mathrm{eq}}}, \frac{\partial \omega}{\partial J_{3}}=\frac{\omega}{J_{3}}, \\
& \frac{\partial \eta}{\partial p^{\prime}}=\frac{1}{\sigma_{\mathrm{eq}}}, \frac{\partial \eta}{\partial \sigma_{\mathrm{eq}}}=-\frac{\eta}{\sigma_{\mathrm{eq}}}, \\
& \frac{\partial u}{\partial \bar{\varepsilon}_{\mathrm{v}}}=\left(1-f_{n}\right), \frac{\partial v}{\partial \bar{\varepsilon}_{\mathrm{v}}}=1, \frac{\partial u}{\partial \bar{\varepsilon}_{\mathrm{m}}}=A_{n}+\frac{\partial A_{n}}{\partial \bar{\varepsilon}_{\mathrm{m}}} \Delta \bar{\varepsilon}_{\mathrm{m}}, \\
& \frac{\partial u}{\partial \bar{\varepsilon}_{\mathrm{d}}}=k_{\omega} \phi_{\eta} \phi_{\omega} f_{n}, \frac{\partial v}{\partial \bar{\varepsilon}_{\mathrm{d}}}=-k_{\omega} \phi_{\eta} \phi_{\omega},
\end{aligned}
$$

and $\frac{\partial p^{\prime}}{\partial \boldsymbol{\sigma}}, \frac{\partial \sigma_{\mathrm{eq}}}{\partial \boldsymbol{\sigma}}$, and $\frac{\partial J_{3}}{\partial \boldsymbol{\sigma}}$ are given by Eqs. (F.2 - F.4). The derivatives of $\lambda$ follows

$$
\frac{\partial \lambda}{\partial \boldsymbol{\sigma}}=\mathbf{0}, \frac{\partial \lambda}{\partial \bar{\varepsilon}_{\mathrm{v}}}=0, \frac{\partial \lambda}{\partial \bar{\varepsilon}_{\mathrm{m}}}=0, \text { and } \frac{\partial \lambda}{\partial \bar{\varepsilon}_{\mathrm{d}}}=\kappa \lambda .
$$

The derivatives of $\chi$ can be estimated from Eq. (H.6) as

$$
\frac{\partial \chi}{\partial \boldsymbol{\sigma}}=\frac{\chi}{3 f} \frac{\partial f}{\partial \boldsymbol{\sigma}}, \frac{\partial \chi}{\partial \bar{\varepsilon}_{\mathrm{v}}}=\frac{\chi}{3 f} \frac{\partial f}{\partial \bar{\varepsilon}_{\mathrm{v}}}, \frac{\partial \chi}{\partial \bar{\varepsilon}_{\mathrm{m}}}=\frac{\chi}{3 f} \frac{\partial f}{\partial \bar{\varepsilon}_{\mathrm{m}}} \text {, and } \frac{\partial \chi}{\partial \bar{\varepsilon}_{\mathrm{d}}}=\frac{\chi}{3 f} \frac{\partial f}{\partial \bar{\varepsilon}_{\mathrm{d}}}+\frac{\chi}{3 \lambda} \frac{\partial \lambda}{\partial \bar{\varepsilon}_{\mathrm{d}}} .
$$


Appendix H.2. Void evolution during internal necking phase

Evolution laws (79) must be integrated in the time interval $\left[t_{n}, t_{n+1}\right]$. The two first equations of the system (79) can be rewritten by

$$
\left\{\begin{array}{l}
\frac{\dot{\chi}}{\chi}=\frac{3}{4} \frac{\lambda}{\chi W}\left(\frac{3}{2 \chi^{2}}-1\right) \dot{\bar{\varepsilon}}_{\mathrm{d}} \\
\frac{\dot{W}}{W}=\frac{3}{4} \frac{\lambda}{\chi W}\left(3-\frac{3}{2 \chi^{2}}\right) \dot{\bar{\varepsilon}}_{\mathrm{d}} .
\end{array}\right.
$$

Defining $Z=\chi W$, one has

$$
\dot{Z}=\frac{3}{2} \lambda \dot{\bar{\varepsilon}}_{\mathrm{d}}
$$

Using the third equation of the system (79), this last equation can be rewritten as

$$
\dot{Z}=\frac{3}{2 \kappa} \dot{\lambda}
$$

The first equation of the system (H.18) leads to

$$
\frac{2 \chi}{3-2 \chi^{2}} \dot{\chi}=\frac{3}{4} \frac{\lambda}{Z} \dot{\bar{\varepsilon}}_{\mathrm{d}}=\frac{1}{2} \frac{\dot{Z}}{Z} .
$$

With respect to the values at the previous time step $\mathbf{Y}_{n}=\left[\begin{array}{llll}f_{n} & \chi_{n} & W_{n} & \lambda_{n}\end{array}\right]^{T}$, the values $\mathbf{Y}=\left[\begin{array}{llll}f & \chi & W & \lambda\end{array}\right]^{T}$ at time $t$ are estimated as follows:

- The value of $\lambda$ using Eq. (H.5):

$$
\lambda=\lambda_{n} \exp \left(\kappa \Delta \bar{\varepsilon}_{\mathrm{d}}\right) .
$$

- The value of $Z$ using Eq. (H.20):

$$
Z=Z_{n}+\frac{3}{2 \kappa}\left(\lambda-\lambda_{n}\right)
$$

where $Z_{n}=\chi_{n} W_{n}$.

- The value of $\chi$ can be estimated from Eq. (H.21) by integrating from $t_{n}$ to $t$, leading to

$$
\chi=\sqrt{\frac{3}{2}-\frac{Z_{n}}{Z}\left(\frac{3}{2}-\chi_{n}^{2}\right)} .
$$

- The value of $W: W=\frac{Z}{\chi}$.

- The value of $f$ using Eq. (40). 
Finally, the derivatives $\frac{\partial \mathbf{Y}}{\partial \boldsymbol{\sigma}}=\mathbf{0}, \frac{\partial \mathbf{Y}}{\partial \bar{\varepsilon}_{\mathrm{v}}}=\mathbf{0}, \frac{\partial \mathbf{Y}}{\partial \bar{\varepsilon}_{\mathrm{m}}}=\mathbf{0}$, and $\frac{\partial \mathbf{Y}}{\partial \bar{\varepsilon}_{\mathrm{d}}}$ are obtained as

$$
\begin{aligned}
\frac{\partial \chi}{\partial \bar{\varepsilon}_{\mathrm{d}}} & =\frac{3}{4} \frac{\lambda}{W}\left(\frac{3}{2 \chi^{2}}-1\right), \\
\frac{\partial W}{\partial \bar{\varepsilon}_{\mathrm{d}}} & =\frac{9}{4} \frac{\lambda}{\chi}\left(1-\frac{1}{2 \chi^{2}}\right), \\
\frac{\partial \lambda}{\partial \bar{\varepsilon}_{\mathrm{d}}} & =\kappa \lambda, \text { and } \\
\frac{\partial f}{\partial \bar{\varepsilon}_{\mathrm{d}}} & =f\left(\frac{3}{\chi} \frac{\partial \chi}{\partial \bar{\varepsilon}_{\mathrm{d}}}+\frac{1}{W} \frac{\partial W}{\partial \bar{\varepsilon}_{\mathrm{d}}}-\frac{1}{\lambda} \frac{\partial \lambda}{\partial \bar{\varepsilon}_{\mathrm{d}}}\right) .
\end{aligned}
$$

Appendix H.3. Void evolution during shear driven coalescence phase

Evolution laws (88) must be integrated in the time interval $\left[t_{n}, t_{n+1}\right]$. Only the second equation of the system (88) will be used leading to

$$
\begin{aligned}
& f=f_{n}, \\
& \chi=\chi_{n}+K_{\chi} \Delta \bar{\varepsilon}_{\mathrm{d}}, \\
& W=W_{n}, \text { and } \\
& \lambda=\frac{\chi^{3} W}{3 \gamma f} .
\end{aligned}
$$

Finally, the derivatives of $\mathbf{Y} \operatorname{read} \frac{\partial \mathbf{Y}}{\partial \boldsymbol{\sigma}}=\mathbf{0}, \frac{\partial \mathbf{Y}}{\partial \bar{\varepsilon}_{\mathrm{v}}}=\mathbf{0}, \frac{\partial \mathbf{Y}}{\partial \bar{\varepsilon}_{\mathrm{m}}}=\mathbf{0}$, and $\frac{\partial \mathbf{Y}}{\partial \bar{\varepsilon}_{\mathrm{d}}}$ is obtained as follows

$$
\begin{aligned}
& \frac{\partial f}{\partial \bar{\varepsilon}_{\mathrm{d}}}=0, \\
& \frac{\partial \chi}{\partial \bar{\varepsilon}_{\mathrm{d}}}=K_{\chi}, \\
& \frac{\partial W}{\partial \bar{\varepsilon}_{\mathrm{d}}}=0, \text { and } \\
& \frac{\partial \lambda}{\partial \bar{\varepsilon}_{\mathrm{d}}}=\frac{\chi^{2} W}{\gamma f} K_{\chi} .
\end{aligned}
$$

\section{Appendix I. Numerical implementation of the nonlocal multi-surface model}

The numerical implementation of the nonlocal multi-surface model follows the general framework described in Appendix A. Since the explicit forms of the yield surface $\Phi_{\mathrm{nl}}$ and of the associative flow normal $\mathbf{N}^{\mathrm{p}}$ exist, the derivatives of $\Phi_{\mathrm{nl}}$ and of $\mathbf{N}^{\mathrm{p}}$ with respect to $\boldsymbol{\sigma}$, $\sigma_{\mathrm{Y}}$, and $\mathbf{Y}$, can be obtained in a closed form as detailed in Appendix F. The void evolution laws following Eqs. $(66,79,88)$ are integrated during the time step $\left[t_{n} t_{n+1}\right]$ as detailed in Appendix H. The procedure to integrate the nonlocal multi-surface model is given in Algorithm 1. 
$\overline{\text { Algorithm } 1 \text { Predictor-corrector scheme of the constitutive behavior during the time step }}$ $\left[t_{n} t_{n+1}\right]$.

Input: $\mathbf{F}_{n+1}, \overline{\mathbf{Z}}_{n+1}, \mathbf{Y}_{n}, \mathbf{F}_{n}^{\mathrm{p}}$, and $\mathbf{Z}_{n}$.

Output: $\mathbf{P}_{n+1}, \mathbf{Z}_{n+1}, \mathbf{F}_{n+1}^{\mathrm{p}}, \mathbf{Y}_{n+1}, \frac{\partial \mathbf{P}_{n+1}}{\partial \mathbf{F}_{n+1}}, \frac{\partial \mathbf{P}_{n+1}}{\partial \overline{\mathbf{Z}}_{n+1}}, \frac{\partial \mathbf{Z}_{n+1}}{\partial \mathbf{F}_{n+1}}$, and $\frac{\partial \mathbf{Z}_{n+1}}{\partial \overline{\mathbf{Z}}_{n+1}}$.

(1) Elastic predictor (see Section Appendix A.1): one has $\mathbf{F}_{n+1}^{\mathrm{p}}=\mathbf{F}_{n}^{\mathrm{p}}, \mathbf{Z}_{n+1}=\mathbf{Z}_{n}$, $\boldsymbol{\tau}_{n+1}$ evaluated using Eq. (A.1), and $\mathbf{Y}_{n+1}$ evaluated using Eq. (A.2) depending on the active damage mechanism as described in Appendix $\mathrm{H}$.

(2) Check the onset of plasticity with the yield function $\Phi_{\mathrm{e}}$ defined in Eq. (90): if $\Phi_{\mathrm{e}} \leq 0$ then go to (4); end

(3) Plastic corrector (see Section Appendix A.2):

(i) initialize $\Delta \mathbf{E}^{p}=\mathbf{0}, \Delta \mu=0$, and $\Delta \varepsilon_{\mathrm{m}}=0$;

(ii) estimate $\boldsymbol{\tau}_{n+1}, \boldsymbol{\sigma}_{n+1}$, and $\sigma_{\mathrm{Y} n+1}$ using Eqs. (A.4, A.5, A.6) respectively;

(iii) estimate $\mathbf{Y}_{n+1}$ using Eq. (A.7) depending on the active damage mechanism as described in Appendix $\mathrm{H}$;

(iv) estimate $\mathbf{r}$ following Eq. (A.11);

(v) check convergence: if $\|\mathbf{r}\|<$ tolerance $\left(10^{-6}-10^{-10}\right)$ then go to (4); end

(vi) update the unknowns:

(a) estimate the Jacobian following Eq. (A.15);

(b) update $\Delta \mathbf{E}^{p}, \Delta \mu$, and $\Delta \varepsilon_{\mathrm{m}}$ using Eq. (A.14);

(c) update $\mathbf{Z}_{n+1}$ using Eqs. (A.18);

(d) go to (ii).

(4) Final step (see Section Appendix A.3):

(i) estimate $\mathbf{P}_{n+1}$ using Eq. (A.20);

(ii) estimate $\frac{\partial \mathbf{P}_{n+1}}{\partial \mathbf{F}_{n+1}}, \frac{\partial \mathbf{P}_{n+1}}{\partial \mathbf{Z}_{n+1}}, \frac{\partial \mathbf{Z}_{n+1}}{\partial \mathbf{F}_{n+1}}, \frac{\partial \mathbf{Z}_{n+1}}{\partial \mathbf{Z}_{n+1}}$ as detailed in Appendix D. 


\section{Appendix J. Path following technique for nonlinear finite element analyses}

When dealing with problems involving material softening, the load drops fast under strain localization and the convergence of a resolution method based on a prescribed force or displacement cannot be guaranteed, and totally fails if snap-back occurs. In order to improve the convergence and to handle the snap-back loading path which possibly occurs, the arc-length path following technique (Riks, 1979, 1992; Geers, 1999) is used.

The weak form of the set of Eqs. $(11,32)$ completed by the boundary conditions $(15,33)$ is obtained by using suitable weight functions defined in the $3+N$ dimensional space, where $N$ is the number of non-local variables (here $N=3$ ). Considering a time step $\left[t_{n} t_{n+1}\right]$, the solution at time $t_{n}$ is known and the problem is solved at time $t_{n+1}$. The weak form is stated as finding $\left(\mathbf{u}, \bar{Z}_{k}, k=1, \ldots, N\right) \in\left[C^{0}\left(B_{0}\right)\right]^{3+N}$ such that

$$
\begin{aligned}
& \int_{B_{0}} \delta \mathbf{u} \cdot \mathbf{P} \cdot \nabla_{0} d V=0, \text { and } \\
& \int_{B_{0}}\left[\bar{Z}_{k}-Z_{k}-\nabla_{0} \cdot\left(\mathbf{C}_{k} \cdot \nabla_{0} \bar{Z}_{k}\right)\right] \delta \bar{Z}_{k} d V=0 \\
& \forall\left(\delta \mathbf{u}, \delta \bar{Z}_{k}, k=1, \ldots, N\right) \text { kinematically admissible. }
\end{aligned}
$$

Applying the divergence theorem and using the boundary conditions, the weak form (J.1) becomes

$$
\begin{aligned}
& \int_{B_{0}} \mathbf{P}:\left(\delta \mathbf{u} \otimes \nabla_{0}\right) d V=\int_{\partial_{N} B_{0}} \overline{\mathbf{T}} \cdot \delta \mathbf{u} d A, \text { and } \\
& \int_{B_{0}}\left(\bar{Z}_{k} \delta \bar{Z}_{k}+\nabla_{0} \bar{Z}_{k} \cdot \mathbf{C}_{k} \cdot \nabla_{0} \delta \bar{Z}_{k}-Z_{k} \delta \bar{Z}_{k}\right) d V=\mathbf{0},
\end{aligned}
$$

with $k=1, \ldots, N$.

The displacement field and non-local variables are then approximated in each element $\Omega_{0}^{e}$ with the shape function matrix $\mathbf{N}$, such that

$$
\begin{aligned}
\mathbf{u}\left(\Omega_{0}^{e}\right) & =\mathbf{N}\left(\Omega_{0}^{e}\right) \mathbf{u}^{e}, \\
\bar{Z}_{k}\left(\Omega_{0}^{e}\right) & =\mathbf{N}\left(\Omega_{0}^{e}\right) \boldsymbol{\Phi}_{k}^{e}, \\
\delta \mathbf{u}\left(\Omega_{0}^{e}\right) & =\mathbf{N}\left(\Omega_{0}^{e}\right) \delta \mathbf{u}^{e}, \text { and } \\
\delta \bar{Z}_{k}\left(\Omega_{0}^{e}\right) & =\mathbf{N}\left(\Omega_{0}^{e}\right) \delta \boldsymbol{\Phi}_{k}^{e} .
\end{aligned}
$$

The deformation gradient and the gradient of the non-local variables are directly deduced as

$$
\begin{aligned}
\mathbf{F}\left(\Omega_{0}^{e}\right) & =\mathbf{B}\left(\Omega_{0}^{e}\right) \mathbf{u}^{e}, \text { and } \\
\nabla_{0} \bar{Z}_{k}\left(\Omega_{0}^{e}\right) & =\mathbf{B}\left(\Omega_{0}^{e}\right) \mathbf{\Phi}_{k}^{e},
\end{aligned}
$$

where $\mathbf{B}$ is the matrix of the gradients of shape function $\mathbf{N}$. Using the finite element 
Algorithm 2 Implementation of the arc-length path following technique from the beginning $\left(t=t_{0}\right)$ to the end of the applied loading $\left(t=t_{\text {end }}\right)$.

(1) Set initial condition:

(i) set $n=0$ and $t=t_{0}$;

(ii) initialize $\mathbf{U}_{0}=\mathbf{U}\left(t_{0}\right)$ and $a_{0}=0$;

(iii) compute the external reference force $\mathbf{Q}^{\text {ext }}$;

(iv) for integration point do

initialize $\mathbf{F}_{0}=\mathbf{F}\left(t_{0}\right), \overline{\mathbf{Z}}_{0}=\overline{\mathbf{Z}}\left(t_{0}\right), \mathbf{P}_{0}=\mathbf{P}\left(t_{0}\right), \mathbf{Z}_{0}=\mathbf{Z}\left(t_{0}\right), \mathbf{Y}_{0}=\mathbf{Y}\left(t_{0}\right)$, and $\mathbf{F}_{0}^{\mathrm{p}}=\mathbf{F}^{\mathrm{p}}\left(t_{0}\right)$

end

(2) Start from previous converged solution:

(i) set $t=t_{n+1}$;

(ii) set $\mathbf{U}_{n+1}=\mathbf{U}_{n}$ and $a_{n+1}=a_{n}$;

(3) Impose $\Delta s_{n+1}$ in Eq. (J.15);

(4) Apply boundary conditions;

(5) Check convergence of Eq. (J.15): if convergence then go to (11); end

(6) Evaluate constitutive behavior:

for integration point do

compute $\mathbf{F}_{n+1}, \overline{\mathbf{Z}}_{n+1}$, and $\boldsymbol{\nabla}_{0} \overline{\mathbf{Z}}_{n+1}$

compute $\mathbf{P}_{n+1}, \mathbf{Z}_{n+1}, \frac{\partial \mathbf{P}_{n+1}}{\partial \mathbf{F}_{n+1}}, \frac{\partial \mathbf{P}_{n+1}}{\partial \mathbf{Z}_{n+1}}, \frac{\partial \mathbf{Z}_{n+1}}{\partial \mathbf{F}_{n+1}}, \frac{\partial \mathbf{Z}_{n+1}}{\partial \overline{\mathbf{Z}}_{n+1}}$ using Algorithm 1;

end

(7) Assemble F $^{\text {int }}$ in Eq. (J.15);

(8) Check convergence of Eq. (J.15): if convergence then go to (10); end

(9) Correct $\mathbf{U}_{n+1}$ and $a_{n+1}$ :

(i) solve linearized form of Eq. (J.15);

(ii) go to (5);

(10) Check the onset of coalescence:

for integration point do

select the void shape evolution laws from conditions $(96,97)$;

end

(11) if $t<t_{\text {end }}$ then $n=n+1$ and go to (2); else go to (12); end

(12) Finish 
approximations (J.3-J.6), the weak form (J.2) is equivalent to the non-linear system of equations

$$
\mathbf{F}^{\text {int }}-\mathbf{F}^{\mathrm{ext}}=\mathbf{0}
$$

where the force vectors are given by

$$
\mathbf{F}^{\text {int }}=\left[\begin{array}{c}
\mathbf{F}_{u}^{\mathrm{int}} \\
\mathbf{F}_{\bar{Z}_{1}} \\
\vdots \\
\mathbf{F}_{\bar{Z}_{N}}
\end{array}\right] \text { and } \mathbf{F}^{\mathrm{ext}}=\left[\begin{array}{c}
\mathbf{F}_{u}^{\mathrm{ext}} \\
\mathbf{0} \\
\vdots \\
\mathbf{0}
\end{array}\right]
$$

in which

$$
\begin{aligned}
\mathbf{F}_{u}^{\mathrm{int}} & =\int_{B_{0}} \mathbf{B}^{T} \mathbf{P} d V, \\
\mathbf{F}_{u}^{\mathrm{ext}} & =\int_{\partial_{N} B_{0}} \mathbf{N}^{T} \overline{\mathbf{T}}_{0} d A, \text { and } \\
\mathbf{F}_{\bar{Z}_{k}} & =\int_{B_{0}}\left[\left(\mathbf{N}^{T} \mathbf{N}+\mathbf{B}^{T} \mathbf{C}_{k} \mathbf{B}\right) \boldsymbol{\Phi}_{k} d V-\mathbf{N}^{T} Z_{k}\right] d V,
\end{aligned}
$$

with $k=1, \ldots, N$.

With the use of the arc-length path following method (Riks, 1979, 1992; Geers, 1999), $\mathbf{F}^{\text {ext }}$ in Eq. (J.9) is parameterized as

$$
\mathbf{F}^{\mathrm{ext}}=a \mathbf{Q}^{\mathrm{ext}}
$$

where $\mathbf{Q}^{\text {ext }}$ is the reference external force vector and $a$ is the load factor, which is considered as an additional unknown of the problem. As a result, Eq. (J.9) results in the following arc-length nonlinear equation

$$
\mathbf{R}\left(\mathbf{U}_{n+1}, a_{n+1}\right)=\left[\begin{array}{c}
\mathbf{F}^{\mathrm{int}}\left(\mathbf{U}_{n+1}\right)-a_{n+1} \mathbf{Q}^{\mathrm{ext}} \\
\alpha_{u} \Delta \mathbf{U}_{n+1}^{T} \Delta \mathbf{U}_{n+1}+\Delta a_{n+1}^{2}-\Delta s_{n+1}^{2}
\end{array}\right]=\mathbf{0},
$$

where $n+1$ is the current time index, $\mathbf{U}$ is the unknown vector consisting of the displacement unknowns $\mathbf{u}^{e}$ and nonlocal unknowns $\boldsymbol{\Phi}_{k}^{e}, \Delta \mathbf{U}_{n+1}=\mathbf{U}_{n+1}-\mathbf{U}_{n}, \mathbf{F}^{\text {int }}$ is the internal force vector (J.10), $\Delta a_{n+1}=a_{n+1}-a_{n}, \alpha_{u}$ is the scaling factor, which is used because the units of $\mathbf{U}$ and of $a$ are different, and $\Delta s$ is the arc-length increment. The iterative solution of Eq. (J.15) to find $\mathbf{U}_{n+1}$ and $a_{n+1}$ is detailed in the work of Nguyen and Noels (2014), and sketched in Algorithm 2.

To avoid volumetric locking which possibly occurs for large plastic flow under low stress triaxialities, high-order elements with reduced integration are considered. In the present work, as only plane strain and axisymmetric problems are considered, six-node triangular elements integrated with 3 Gauss points are used. 


\section{References}

Achouri, M., Germain, G., Santo, P.D., Saidane, D., 2013. Experimental characterization and numerical modeling of micromechanical damage under different stress states. Materials \& Design 50, 207 - 222. URL: http://www.sciencedirect.com/science/article/pii/S0261306913001866, doi:https://doi.org/10.1016/j.matdes.2013.02.075.

Aldakheel, F., Wriggers, P., Miehe, C., 2018. A modified gurson-type plasticity model at finite strains: Formulation, numerical analysis and phase-field coupling. Comput. Mech. 62, 815-833. URL: https ://doi .org/10.1007/s00466-017-1530-0, doi:10.1007/s00466-017-1530-0.

Ambati, M., Kruse, R., De Lorenzis, L., 2016. A phase-field model for ductile fracture at finite strains and its experimental verification. Computational Mechanics 57, 149-167. URL: https : //doi .org/10.1007/s00466-015-1225-3, doi:10.1007/s00466-015-1225-3.

Anand, L., Aslan, O., Chester, S.A., 2012. A large-deformation gradient theory for elasticplastic materials: Strain softening and regularization of shear bands. International Journal of Plasticity 3031, 116 - 143. URL: http://www.sciencedirect.com/science/article/pii/S0749641911001665, doi:https://doi.org/10.1016/j.ijplas.2011.10.002.

Andrade, F., de Sá, J.C., Pires, F.A., 2014. Assessment and comparison of non-local integral models for ductile damage. International Journal of Damage Mechanics 23, 261296. URL: https://doi.org/10.1177/1056789513493103, doi:10.1177/1056789513493103, arXiv:https://doi.org/10.1177/1056789513493103.

Bao, Y., Wierzbicki, T., 2004. On fracture locus in the equivalent strain and stress triaxiality space. International Journal of Mechanical Sciences 46, $81 \quad-$ 98. URL: http://www.sciencedirect.com/science/article/pii/S0020740304000360, doi:https://doi.org/10.1016/j.ijmecsci.2004.02.006.

Barsoum, I., Faleskog, J., 2007. Rupture mechanisms in combined tension and shearexperiments. International Journal of Solids and Structures 44, $1768 \quad-\quad 1786$. URL: $\quad$ http://www.sciencedirect.com/science/article/pii/S0020768306003921, doi:https://doi.org/10.1016/j.ijsolstr.2006.09.031. physics and Mechanics of Advanced Materials.

Barsoum, I., Faleskog, J., 2011. Micromechanical analysis on the influence of the lode parameter on void growth and coalescence. International Journal of Solids and Structures 48, 925 - 938. URL: http://www.sciencedirect.com/science/article/pii/S002076831000435X, doi:https://doi.org/10.1016/j.ijsolstr.2010.11.028.

Barsoum, I., Faleskog, J., Pingle, S., 2012. The effect of stress state on ductility in the moderate stress triaxiality regime of medium and high strength steels. International Journal of Mechanical Sciences 65, 203 - 212. URL: http://www.sciencedirect.com/science/article/pii/S0020740312002226, doi:https://doi.org/10.1016/j.ijmecsci.2012.10.003.

Bažant, Z.P., Jirásek, M., 2002. Nonlocal integral formulations of plasticity and damage: survey of progress. Journal of Engineering Mechanics 128, 1119-1149.

Benzerga, A., 2002. Micromechanics of coalescence in ductile fracture. Journal of the Mechanics and Physics of Solids 50, 1331 - $1362 . \quad$ URL: http://www.sciencedirect.com/science/article/pii/S0022509601001259, doi:https://doi.org/10.1016/S0022-5096(01)00125-9.

Benzerga, A., Besson, J., Pineau, A., 2004. Anisotropic ductile fracture: Part ii: theory. Acta Materialia 52, 4639 - 4650. URL: http://www.sciencedirect.com/science/article/pii/S135964540400357X, doi:https://doi.org/10.1016/j.actamat.2004.06.019.

Benzerga, A.A., Besson, J., Batisse, R., Pineau, A., 2002. Synergistic effects of plastic anisotropy and void coalescence on fracture mode in plane strain. Modelling and Simulation in Materials Science and Engineering 10, 73. URL: http://stacks.iop.org/0965-0393/10/i=1/a=306.

Benzerga, A.A., Leblond, J.B., 2010. Ductile fracture by void growth to coalescence, in: Aref, H., van der Giessen, E. (Eds.), Advances in Applied Mechanics. Elsevier. volume 44 of Advances in Applied Mechanics, pp. 169 - 305. URL: http://www.sciencedirect.com/science/article/pii/S006521561044003X, doi:https://doi.org/10.1016/S0065-2156(10)44003-X. 
Benzerga, A.A., Leblond, J.B., 2014. Effective yield criterion accounting for microvoid coalescence. Journal of Applied Mechanics 81, 031009.

Benzerga, A.A., Leblond, J.B., Needleman, A., Tvergaard, V., 2016. Ductile failure modeling. International Journal of Fracture 201, 29-80. URL: https://doi.org/10.1007/s10704-016-0142-6, doi:10.1007/s10704-016-0142-6.

Besson, J., 2009. Damage of ductile materials deforming under multiple plastic or viscoplastic mechanisms. International Journal of Plasticity 25, 2204 2221. URL: http://www.sciencedirect.com/science/article/pii/S0749641909000357, doi:https://doi.org/10.1016/j.ijplas.2009.03.001.

Besson, J., Steglich, D., Brocks, W., $2003 . \quad$ Modeling of plane strain ductile rupture. International Journal of Plasticity $19, \quad 1517 \quad-\quad 1541$. URL: $\quad$ http://www.sciencedirect.com/science/article/pii/S0749641902000220, doi:https://doi.org/10.1016/S0749-6419(02)00022-0.

Chen, J., Yuan, H., 2002. A micro-mechanical damage model based on gradient plasticity: algorithms and applications. International Journal for Numerical Methods in Engineering 54, 399-420.

Chu, C., Needleman, A., 1980. Void nucleation effects in biaxially stretched sheets. Journal of engineering materials and technology 102, 249-256.

De Borst, R., Mühlhaus, H.B., 1992. Gradient-dependent plasticity: formulation and algorithmic aspects. International Journal for Numerical Methods in Engineering 35, 521-539.

De Borst, R., Sluys, L., Muhlhaus, H.B., Pamin, J., 1993. Fundamental issues in finite element analyses of localization of deformation. Engineering computations 10, 99-121.

Dunand, M., Mohr, D., 2011. On the predictive capabilities of the shear modified gurson and the modified mohrcoulomb fracture models over a wide range of stress triaxialities and lode angles. Journal of the Mechanics and Physics of Solids 59, $1374 \quad-$ 1394. URL: http://www.sciencedirect.com/science/article/pii/S0022509611000688, doi:https://doi.org/10.1016/j.jmps.2011.04.006.

Dunand, M., Mohr, D., 2014. Effect of lode parameter on plastic flow localization after proportional loading at low stress triaxialities. Journal of the Mechanics and Physics of Solids 66, 133 - 153. URL: http://www.sciencedirect.com/science/article/pii/S0022509614000180, doi:https://doi.org/10.1016/j.jmps.2014.01.008.

Enakoutsa, K., Leblond, J., Perrin, G., 2007. Numerical implementation and assessment of a phenomenological nonlocal model of ductile rupture. Computer Methods in Applied Mechanics and Engineering 196, 1946 - 1957. URL: http://www.sciencedirect.com/science/article/pii/S004578250600329X, doi:https://doi.org/10.1016/j.cma.2006.10.003.

Engelen, R.A., Geers, M.G., Baaijens, F.P., 2003. Nonlocal implicit gradient-enhanced elastoplasticity for the modelling of softening behaviour. International Journal of Plasticity 19, 403 - 433. URL: http://www.sciencedirect.com/science/article/pii/S0749641901000420, doi:https://doi.org/10.1016/S0749-6419(01)00042-0.

Faleskog, J., Barsoum, I., 2013. Tensiontorsion fracture experimentspart i: Experiments and a procedure to evaluate the equivalent plastic strain. International Journal of Solids and Structures 50, 4241 - 4257. URL: http://www.sciencedirect.com/science/article/pii/S0020768313003454, doi:https://doi.org/10.1016/j.ijsolstr.2013.08.029.

Forest, S., 2009. Micromorphic approach for gradient elasticity, viscoplasticity, and damage. Journal of Engineering Mechanics 135, 117-131. doi:10.1061/(ASCE)0733-9399(2009)135:3(117).

Gao, X., Zhang, G., Roe, C., 2010. A study on the effect of the stress state on ductile fracture. International Journal of Damage Mechanics 19, 75-94. URL: https://doi.org/10.1177/1056789509101917, doi:10.1177/1056789509101917, arXiv:https://doi.org/10.1177/1056789509101917.

Geers, M.G.D., 1999. Enhanced solution control for physically and geometrically non-linear problems. part iicomparative performance analysis. International Journal for Numerical Methods in Engineering 46, 205-230. URL: http://dx.doi.org/10.1002/(SICI) 1097-0207(19990920) 46:2<205: :AID-NME669>3.0.C0;2-S, 
doi:10.1002/(SICI)1097-0207(19990920)46:2;205::AID-NME669;3.0.CO;2-S.

Geers, M.G.D., Peerlings, R.H.J., Brekelmans, W.A.M., de Borst, R., 2000. Phenomenological nonlocal approaches based on implicit gradient-enhanced damage. Acta Mechanica 144, 1-15. URL: https://doi.org/10.1007/BF01181824, doi:10.1007/BF01181824.

Ghahremaninezhad, A., Ravi-Chandar, K., 2013. Ductile failure behavior of polycrystalline al 6061-t6 under shear dominant loading. International Journal of Fracture 180, 23-39. URL: https://doi .org/10.1007/s10704-012-9793-0, doi:10.1007/s10704-012-9793-0.

Gologanu, M., Leblond, J.B., Devaux, J., 1993. Approximate models for ductile metals containing non-spherical voids - case of axisymmetric prolate ellipsoidal cavities. Journal of the Mechanics and Physics of Solids 41, 1723 1754. URL: http://www.sciencedirect.com/science/article/pii/002250969390029F, doi:https://doi.org/10.1016/0022-5096(93)90029-F.

Gologanu, M., Leblond, J.B., Devaux, J., 1994. Approximate models for ductile metals containing nonspherical voids - case of axisymmetric oblate ellipsoidal cavities. Journal of Engineering Materials and Technology 116, 290-297.

Gurson, A.L., 1977. Continuum theory of ductile rupture by void nucleation and growth: Part i - yield criteria and flow rules for porous ductile media. Journal of Engineering Materials and Technology 99, $2-15$.

Gurtin, M.E., Anand, L., 2005. The decomposition $\mathrm{f}=\mathrm{fefp}$, material symmetry, and plastic irrotationality for solids that are isotropic-viscoplastic or amorphous. International Journal of Plasticity 21, 1686 - 1719. URL: http://www.sciencedirect.com/science/article/pii/S0749641904001603, doi:https://doi.org/10.1016/j.ijplas.2004.11.007.

Håkansson, P., Wallin, M., Ristinmaa, M., 2006. Thermomechanical response of non-local porous material. International Journal of Plasticity 22, $2066-2090$. URL: $\quad$ http://www.sciencedirect.com/science/article/pii/S0749641906000623, doi:https://doi.org/10.1016/j.ijplas.2005.08.003. damage and Fracture: Modeling and Experiments.

Haltom, S., Kyriakides, S., Ravi-Chandar, K., 2013. Ductile failure under combined shear and tension. International Journal of Solids and Structures 50, 1507 1522. URL: http://www.sciencedirect.com/science/article/pii/S0020768312005203, doi:https://doi.org/10.1016/j.ijsolstr.2012.12.009.

Hütter, G., Linse, T., Mühlich, U., Kuna, M., 2013. Simulation of ductile crack initiation and propagation by means of a non-local gurson-model. International Journal of Solids and Structures 50, 662 - 671. URL: http://www.sciencedirect.com/science/article/pii/S0020768312004659, doi:https://doi.org/10.1016/j.ijsolstr.2012.10.031.

Hütter, G., Linse, T., Roth, S., Mühlich, U., Kuna, M., 2014. A modeling approach for the complete ductile-brittle transition region: cohesive zone in combination with a non-local gurson-model. International Journal of Fracture 185, 129-153. URL: https://doi.org/10.1007/s10704-013-9914-4, doi:10.1007/s10704-013-9914-4.

Jirásek, M., 1998. Nonlocal models for damage and fracture: Comparison of approaches. International Journal of Solids and Structures 35, 4133 - 4145. URL: $\quad$ http://www.sciencedirect.com/science/article/pii/S0020768397003065, doi:https://doi.org/10.1016/S0020-7683(97)00306-5.

Jirásek, M., Rolshoven, S., 2003. Comparison of integral-type nonlocal plasticity models for strain-softening materials. International Journal of Engineering Science 41, 1553 - 1602. URL: http://www.sciencedirect.com/science/article/pii/S0020722503000272, doi:https://doi.org/10.1016/S0020-7225(03)00027-2. damage and failure analysis of materials.

Keralavarma, S., Benzerga, A., 2010. A constitutive model for plastically anisotropic solids with non-spherical voids. Journal of the Mechanics and Physics of Solids 58, 874 901. URL: http://www.sciencedirect.com/science/article/pii/S0022509610000682, doi:https://doi.org/10.1016/j.jmps.2010.03.007.

Keralavarma, S., Chockalingam, S., 2016. A criterion for void coalescence in 
anisotropic ductile materials. International Journal of Plasticity $82, \quad 159 \quad-\quad 176$. URL: $\quad$ http://www.sciencedirect.com/science/article/pii/S0749641916300274, doi:https://doi.org/10.1016/j.ijplas.2016.03.003.

Kim, J., Gao, X., Srivatsan, T.S., 2004. Modeling of void growth in ductile solids: effects of stress triaxiality and initial porosity. Engineering Fracture Mechanics 71, 379 - 400. URL: http://www.sciencedirect.com/science/article/pii/S0013794403001140, doi:https://doi.org/10.1016/S0013-7944(03)00114-0.

Leblond, J., Perrin, G., Devaux, J., 1994. Bifurcation effects in ductile metals with nonlocal damage. Journal of Applied Mechanics 61, 236-242.

Leclerc, J., Nguyen, V.D., Pardoen, T., Noels, L., 2020. A micromechanics-based non-local damage to crack transition framework for porous elastoplastic solids. International Journal of Plasticity doi:10.1016/j.ijplas.2019.11.010.

Leclerc, J., Wu, L., Nguyen, V.D., Noels, L., 2018. A damage to crack transition model accounting for stress triaxiality formulated in a hybrid nonlocal implicit discontinuous galerkin-cohesive band model framework. International Journal for Numerical Methods in Engineering 113, 374410. URL: https://onlinelibrary.wiley.com/doi/abs/10.1002/nme.5618, doi:10.1002/nme.5618, arXiv:https://onlinelibrary.wiley.com/doi/pdf/10.1002/nme.5618.

Ling, C., Forest, S., Besson, J., Tanguy, B., Latourte, F., 2018. A reduced micromorphic single crystal plasticity model at finite deformations. application to strain localization and void growth in ductile metals. International Journal of Solids and Structures 134, 43 - 69. URL: http://www.sciencedirect.com/science/article/pii/S0020768317304742, doi:https://doi.org/10.1016/j.ijsolstr.2017.10.013.

Linse, T., Hütter, G., Kuna, M., 2012. Simulation of crack propagation using a gradientenriched ductile damage model based on dilatational strain. Engineering Fracture Mechanics 95, 13 - 28. URL: http://www.sciencedirect.com/science/article/pii/S0013794412002809, doi:https://doi.org/10.1016/j.engfracmech.2012.07.004. cracks in Microstructures and Engineering Components.

Miehe, C., Kienle, D., Aldakheel, F., Teichtmeister, S., 2016. Phase field modeling of fracture in porous plasticity: A variational gradient-extended eulerian framework for the macroscopic analysis of ductile failure. Computer Methods in Applied Mechanics and Engineering 312, 3 - 50. URL: http://www.sciencedirect.com/science/article/pii/S0045782516305412, doi:https://doi.org/10.1016/j.cma.2016.09.028. phase Field Approaches to Fracture.

Moran, B., Ortiz, M., Shih, C., 1990. Formulation of implicit finite element methods for multiplicative finite deformation plasticity. International Journal for Numerical Methods in Engineering 29, 483-514.

Morin, L., Leblond, J.B., Benzerga, A.A., Kondo, D., 2016. A unified criterion for the growth and coalescence of microvoids. Journal of the Mechanics and Physics of Solids 97, 19 - 36. URL: http://www.sciencedirect.com/science/article/pii/S0022509616300436, doi:https://doi.org/10.1016/j.jmps.2016.01.013. sI:Pierre Suquet Symposium.

Nahshon, K., Hutchinson, J., 2008. Modification of the gurson model for shear failure. European Journal of Mechanics - A/Solids 27, 1 - 17. URL: $\quad$ http://www.sciencedirect.com/science/article/pii/S0997753807000721, doi:https://doi.org/10.1016/j.euromechsol.2007.08.002.

Needleman, A., Tvergaard, V., 1984. An analysis of ductile rupture in notched bars. Journal of the Mechanics and Physics of Solids 32, $461 \quad-\quad 490$. URL: http://www.sciencedirect.com/science/article/pii/0022509684900310, doi:https://doi.org/10.1016/0022-5096(84)90031-0.

Nguyen, V.D., Lani, F., Pardoen, T., Morelle, X., Noels, L., 2016. A large strain hyperelastic viscoelastic-viscoplastic-damage constitutive model based on a multi-mechanism non-local damage continuum for amorphous glassy polymers. International Journal of Solids and Structures 96, 192 - 216. URL: http://www.sciencedirect.com/science/article/pii/S0020768316301238, doi:https://doi.org/10.1016/j.ijsolstr.2016.06.008. 
Nguyen, V.D., Noels, L., 2014. Computational homogenization of cellular materials. International Journal of Solids and Structures 51, $2183 \quad-\quad 2203$. URL: $\quad$ http://www.sciencedirect.com/science/article/pii/S0020768314000778, doi:https://doi.org/10.1016/j.ijsolstr.2014.02.029.

Nielsen, K.L., Tvergaard, V., 2010. Ductile shear failure or plug failure of spot welds modelled by modified gurson model. Engineering Fracture Mechanics 77, $1031 \quad-$ 1047. URL: http://www.sciencedirect.com/science/article/pii/S0013794410001128, doi:https://doi.org/10.1016/j.engfracmech.2010.02.031.

Papasidero, J., Doquet, V., Mohr, D., 2014. Determination of the effect of stress state on the onset of ductile fracture through tension-torsion experiments. Experimental Mechanics 54, 137-151. URL: https : //doi .org/10.1007/s11340-013-9788-4, doi:10.1007/s11340-013-9788-4.

Pardoen, T., 2006. Numerical simulation of low stress triaxiality ductile fracture. Computers \& Structures 84, 1641 - 1650. URL: http://www.sciencedirect.com/science/article/pii/S0045794906001659, doi:https://doi.org/10.1016/j.compstruc.2006.05.001.

Pardoen, T., Hutchinson, J., 2000. An extended model for void growth and coalescence. Journal of the Mechanics and Physics of Solids 48, 2467 - 2512. URL: http://www.sciencedirect.com/science/article/pii/S0022509600000193, doi:https://doi.org/10.1016/S0022-5096(00)00019-3.

Pardoen, T., Hutchinson, J., $2003 . \quad$ Micromechanics-based model for trends in toughness of ductile metals. Acta Materialia 51, 133 - 148 URL: http://www.sciencedirect.com/science/article/pii/S1359645402003865, doi:https://doi.org/10.1016/S1359-6454(02)00386-5.

Peerlings, R., Geers, M., de Borst, R., Brekelmans, W., 2001. A critical comparison of nonlocal and gradient-enhanced softening continua. International Journal of Solids and Structures 38, 7723 - 7746. URL: http://www.sciencedirect.com/science/article/pii/S0020768301000877, doi:https://doi.org/10.1016/S0020-7683(01)00087-7.

Peerlings, R., Massart, T., Geers, M., 2004. A thermodynamically motivated implicit gradient damage framework and its application to brick masonry cracking. Computer Methods in Applied Mechanics and Engineering 193, 3403 3417. URL: http://www.sciencedirect.com/science/article/pii/S0045782504001380, doi:https://doi.org/10.1016/j.cma.2003.10.021. computational Failure Mechanics.

Peerlings, R.H.J., De Borst, R., Brekelmans, W.A.M., De Vree, J.H.P., 1996. Gradient enhanced damage for quasi-brittle materials. International Journal for Numerical Methods in Engineering 39, 3391-3403. URL: http: //www. scopus. com/inward/record. url?eid=2-s2.0-0030267284\&partnerID=tZ0tx3y1.

Pineau, A., Benzerga, A., Pardoen, T., 2016. Failure of metals i: Brittle and ductile fracture. Acta Materialia 107, 424 - 483. URL: http://www.sciencedirect.com/science/article/pii/S1359645415301403, doi:https://doi.org/10.1016/j.actamat.2015.12.034.

Poh, L.H., Sun, G., 2017. Localizing gradient damage model with decreasing interactions. International Journal for Numerical Methods in Engineering 110, 503-522. URL: https://onlinelibrary.wiley.com/doi/abs/10.1002/nme.5364, doi:10.1002/nme.5364, arXiv:https://onlinelibrary.wiley.com/doi/pdf/10.1002/nme.5364.

Ramaswamy, S., Aravas, N., 1998. Finite element implementation of gradient plasticity models part ii: Gradient-dependent evolution equations. Computer Methods in Applied Mechanics and Engineering 163, 33 - 53. URL: http://www.sciencedirect.com/science/article/pii/S0045782598000279, doi:https://doi.org/10.1016/S0045-7825(98)00027-9.

Reddi, D., Areej, V., Keralavarma, S., 2019. Ductile failure simulations using a multisurface coupled damage-plasticity model. International Journal of Plasticity 118, $190 \quad-$ 214. URL: http://www.sciencedirect.com/science/article/pii/S0749641918307149, doi:https://doi.org/10.1016/j.ijplas.2019.02.007.

Reusch, F., Hortig, C., Svendsen, B., 2008. Nonlocal modeling and simulation of ductile damage and failure in metal matrix composites. Journal of Engineering Materials and Technology 130, 021009. 
Reusch, F., Svendsen, B., Klingbeil, D., 2003a. Local and non-local gurson-based ductile damage and failure modelling at large deformation. European Journal of Mechanics - A/Solids 22, 779 - 792. URL: http://www.sciencedirect.com/science/article/pii/S0997753803000706, doi:https://doi.org/10.1016/S0997-7538(03)00070-6.

Reusch, F., Svendsen, B., Klingbeil, D., 2003b. A non-local extension of gursonbased ductile damage modeling. Computational Materials Science 26, 219 229. URL: http://www.sciencedirect.com/science/article/pii/S0927025602004020, doi:https://doi.org/10.1016/S0927-0256(02)00402-0. 11th International Workshop on Computational Mechanics of Materials.

Riks, E., 1979. An incremental approach to the solution of snapping and buckling problems. International Journal of Solids and Structures 15, 529 - 551. URL: $\quad$ http://www.sciencedirect.com/science/article/pii/0020768379900817, doi:http://dx.doi.org/10.1016/0020-7683(79)90081-7.

Riks, E., 1992. On formulations of path-following techniques for structural stability analysis. NASA STI/Recon Technical Report N 931, 16346-+.

Scheyvaerts, F., Onck, P., Tekoğlu, C., Pardoen, T., 2011. The growth and coalescence of ellipsoidal voids in plane strain under combined shear and tension. Journal of the Mechanics and Physics of Solids 59, 373 - 397. URL: http://www.sciencedirect.com/science/article/pii/S0022509610002061, doi:https://doi.org/10.1016/j.jmps.2010.10.003.

Scheyvaerts, F., Pardoen, T., Onck, P., 2010. A new model for void coalescence by internal necking. International Journal of Damage Mechanics 19, 95-126. URL: https://doi.org/10.1177/1056789508101918, doi:10.1177/1056789508101918, arXiv:https://doi.org/10.1177/1056789508101918.

Seidenfuss, M., Samal, M., Roos, E., 2011. On critical assessment of the use of local and nonlocal damage models for prediction of ductile crack growth and crack path in various loading and boundary conditions. International Journal of Solids and Structures 48, 3365 - 3381. URL: http://www.sciencedirect.com/science/article/pii/S002076831100285X, doi:https://doi.org/10.1016/j.ijsolstr.2011.08.006.

Springmann, M., Kuna, M., 2005. Identification of material parameters of the gursontvergaardneedleman model by combined experimental and numerical techniques. Computational Materials Science 33, 501 - 509. URL: http://www.sciencedirect.com/science/article/pii/S0927025605000169, doi:https://doi.org/10.1016/j.commatsci.2005.02.002.

Tekoğlu, C., 2014. Representative volume element calculations under constant stress triaxiality, lode parameter, and shear ratio. International Journal of Solids and Structures 51, 4544 - 4553. URL: http://www.sciencedirect.com/science/article/pii/S0020768314003424, doi:https://doi.org/10.1016/j.ijsolstr.2014.09.001.

Tekoğlu, C., Hutchinson, J.W., Pardoen, T., 2015. On localization and void coalescence as a precursor to ductile fracture. Philosophical Transactions of the Royal Society A: Mathematical, Physical and Engineering Sciences 373, 20140121. URL: $\quad$ https://royalsocietypublishing.org/doi/abs/10.1098/rsta.2014.0121, doi:10.1098/rsta.2014.0121, arXiv:https://royalsocietypublishing.org/doi/pdf/10.1098/rsta.2014.0121.

Tekoğlu, C., Leblond, J.B., Pardoen, T., 2012. A criterion for the onset of void coalescence under combined tension and shear. Journal of the Mechanics and Physics of Solids 60, 1363 - 1381. URL: http://www.sciencedirect.com/science/article/pii/S0022509612000373, doi:https://doi.org/10.1016/j.jmps.2012.02.006.

Thomason, P., 1985a. A three-dimensional model for ductile fracture by the growth and coalescence of microvoids. Acta Metallurgica 33, 1087 - 1095. URL: $\quad$ http://www.sciencedirect.com/science/article/pii/0001616085902020, doi:https://doi.org/10.1016/0001-6160(85)90202-0.

Thomason, P., 1985b. Three-dimensional models for the plastic limit-loads at incipient failure of the intervoid matrix in ductile porous solids. Acta Metallurgica 33, 1079 1085. URL: http://www.sciencedirect.com/science/article/pii/0001616085902019, 
doi:https://doi.org/10.1016/0001-6160(85)90201-9.

Torki, M., Benzerga, A., Leblond, J.B., 2015. On void coalescence under combined tension and shear. Journal of Applied Mechanics 82, 071005.

Torki, M., Tekoğlu, C., Leblond, J.B., Benzerga, A., 2017. Theoretical and numerical analysis of void coalescence in porous ductile solids under arbitrary loadings. International Journal of Plasticity 91, 160 - 181. URL: http://www.sciencedirect.com/science/article/pii/S0749641916302595, doi:https://doi.org/10.1016/j.ijplas.2017.02.011.

Torki, M.E., 2019. A unified criterion for void growth and coalescence under combined tension and shear. International Journal of Plasticity 119, 57 84. URL: http://www.sciencedirect.com/science/article/pii/S0749641918303152, doi:https://doi.org/10.1016/j.ijplas.2019.02.002.

Torki, M.E., Benzerga, A.A., 2018. A mechanism of failure in shear bands. Extreme Mechanics Letters 23, 67 - 71. URL: http://www.sciencedirect.com/science/article/pii/S2352431618300890, doi:https://doi.org/10.1016/j.eml.2018.06.008.

Tvergaard, V., Needleman, A., 1984. Analysis of the cup-cone fracture in a round tensile bar. Acta Metallurgica 32, 157 - 169. URL: http://www. sciencedirect.com/science/article/pii/000161608490213X, doi:https://doi.org/10.1016/0001-6160(84)90213-X.

Tvergaard, V., Needleman, A., 1995. Effects of nonlocal damage in porous plastic solids. International Journal of Solids and Structures 32, 1063 - 1077. URL: $\quad$ http://www.sciencedirect.com/science/article/pii/002076839400185Y, doi:https://doi.org/10.1016/0020-7683(94)00185-Y.

Vadillo, G., Reboul, J., Fernndez-Sez, J., 2016. A modified gurson model to account for the influence of the lode parameter at high triaxialities. European Journal of Mechanics - A/Solids 56, 31 - 44. URL: http://www.sciencedirect.com/science/article/pii/S099775381500131X, doi:https://doi.org/10.1016/j.euromechsol.2015.09.010.

Wu, L., Becker, G., Noels, L., 2014. Elastic damage to crack transition in a coupled non-local implicit discontinuous galerkin/extrinsic cohesive law framework. Computer Methods in Applied Mechanics and Engineering 279, 379 409. URL: http://www.sciencedirect.com/science/article/pii/S0045782514002175, doi:https://doi.org/10.1016/j.cma.2014.06.031.

Wu, L., Noels, L., Adam, L., Doghri, I., 2013. An implicit-gradient-enhanced incremental-secant mean-field homogenization scheme for elasto-plastic composites with damage. International Journal of Solids and Structures 50, 3843 3860. URL: http://www.sciencedirect.com/science/article/pii/S0020768313003028, doi:https://doi.org/10.1016/j.ijsolstr.2013.07.022.

Xue, L., 2008. Constitutive modeling of void shearing effect in ductile fracture of porous materials. Engineering Fracture Mechanics 75, 3343 - 3366 . URL: $\quad$ http://www.sciencedirect.com/science/article/pii/S0013794407003220, doi:https://doi.org/10.1016/j.engfracmech.2007.07.022. local Approach to Fracture (19862006): Selected papers from the 9th European Mechanics of Materials Conference.

Xue, Z., Pontin, M., Zok, F., Hutchinson, J., 2010. Calibration procedures for a computational model of ductile fracture. Engineering Fracture Mechanics 77, 492 509. URL: http://www.sciencedirect.com/science/article/pii/S0013794409003233, doi:https://doi.org/10.1016/j.engfracmech.2009.10.007.

Zhang, Y., Lorentz, E., Besson, J., 2018. Ductile damage modelling with locking-free regularised gtn model. International Journal for Numerical Methods in Engineering 113, 18711903. URL: https://onlinelibrary.wiley.com/doi/abs/10.1002/nme.5722, doi:10.1002/nme.5722, arXiv:https://onlinelibrary.wiley.com/doi/pdf/10.1002/nme.5722.

Zhang, Z., Thaulow, C., degrd, J., 2000. A complete gurson model approach for ductile fracture. Engineering Fracture Mechanics 67, 155 - 168. URL: $\quad$ http://www.sciencedirect.com/science/article/pii/S0013794400000552, 
doi:https://doi.org/10.1016/S0013-7944(00)00055-2.

Zhu, Y., Engelhardt, M.D., Kiran, R., 2018. Combined effects of triaxiality, lode parameter and shear stress on void growth and coalescence. Engineering Fracture Mechanics 199, 410 - 437. URL: http://www.sciencedirect.com/science/article/pii/S0013794418300766, doi:https://doi.org/10.1016/j.engfracmech.2018.06.008.

Zybell, L., Hütter, G., Linse, T., Mühlich, U., Kuna, M., 2014. Size effects in ductile failure of porous materials containing two populations of voids. European Journal of Mechanics - A/Solids 45, 8 - 19. URL: http://www.sciencedirect.com/science/article/pii/S0997753813001411, doi:https://doi.org/10.1016/j.euromechsol.2013.11.006. 